\title{
Robotic Pipeline Wall Thickness Evaluation for Dense NDT Inspection
}

\author{
Jaime Valls Miro, Nalika Ulapane, Lei Shi, Dave Hunt and Michael Behrens \\ Centre for Autonomous Systems, University of Technology Sydney, \\ \{jaime.vallsmiro, nalika.ulapane, lei.shi, dave.hunt, michael.behrens\}@uts.edu.au
}

\begin{abstract}
This paper addresses automated mapping of the remaining wall thickness of metallic pipelines in the field by means of an inspection robot equipped with Non-Destructive Testing (NDT) sensing. Set in the context of condition assessment of critical infrastructure, the integrity of arbitrary sections in the conduit is derived with a bespoke robot kinematic configuration that allows dense pipe wall thickness discrimination in circumferential and longitudinal direction via NDT sensing with guaranteed sensing lift-off (offset of the sensor from pipe wall) to the pipe wall, an essential barrier to overcome in cement-lined water pipelines. A tailored covariance function for pipeline cylindrical structures within the context of a Gaussian Processes has also been developed to regress missing sensor data incurred by a sampling strategy followed in the field to speed up the inspection times given the slow response of the PEC electromagnetic sensor proposed. The data gathered represents not only a visual understanding of the condition of the pipe for asset managers, but also constitutes a quantative input to a remaining-life calculation that defines the likelihood of the pipeline for future renewal or repair. Results are presented from deployment of the robotic device on a series of pipeline inspections which demonstrate the feasibility of the device and sensing configuration to provide meaningful $2.5 \mathrm{D}$ geometric maps.
\end{abstract}

\section{Motivation - A Taxonomy of NDT Inspection Techniques}

Non-Destructive Testing (NDT) or Evaluation (NDE) is extensively employed by the energy and water industry to assess the integrity of their network assets, particularly their larger and most critical conduits (generally refered to as those larger than $350 \mathrm{~mm}$ in diameter), in their decision-making process leading their renewal/repair/rehabilitation programs. The key advantage of NDT/NDE is that the structure of the asset is not compromised in estimating its condition.

The sensing modality to use is strongly influenced by the material of the asset. Grey Cast Iron (CI) pipelines remain the bulk of the buried critical water infrastructure in the developed world as that was the material of choice for mass production with the advent of the Industrial Revolution in the middle of the 18th century (alongside its less brittle relative of Ductile Iron since the nineteen fifties), until carbon steel, asbestos cement or plastic pipelines (PVC) amongst other materials made them redundant over the years. The non-homogeneity of the CI produce means that sensing techniques widely employed in the (mild) Carbon Steel networks in the energy pipeline sector, such as ultrasonics or electromagnetic acoustic transducers (EMAT), are inadequate for CI, and the underlying techniques of most commercial propositions for CI are instead based on either magnetics (e.g. Magnetic Flux Leakage (MFL), Pulsed Eddy Currents (PEC) and Remote Field Eddy Currents (RFEC)), or the study of the propagation of pressure waves in the pipeline and/or fluid. 


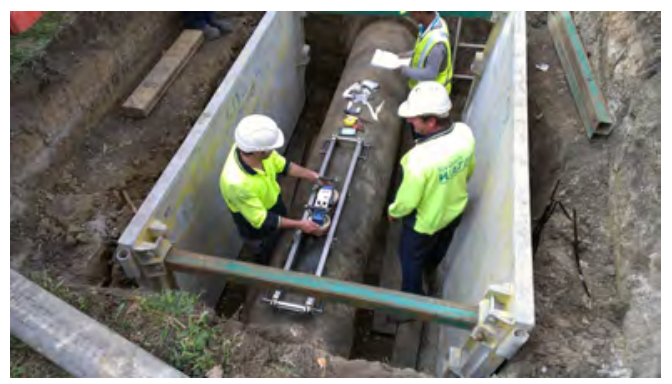

(a) Externa locall inspection tool.
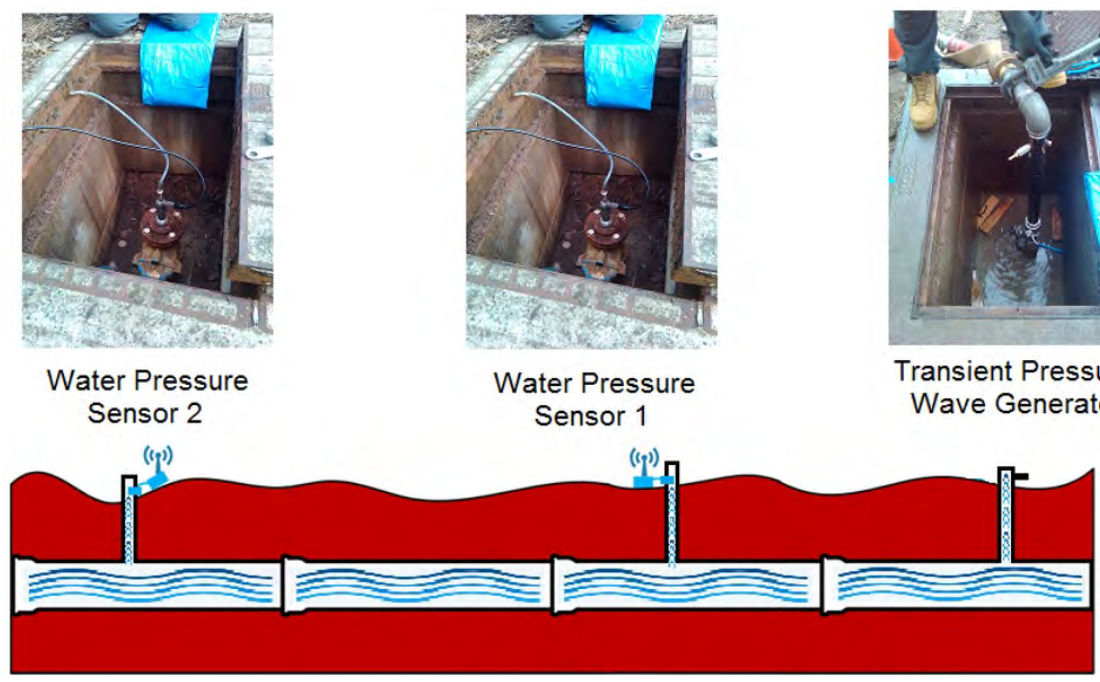

(c) An external averaging tool.

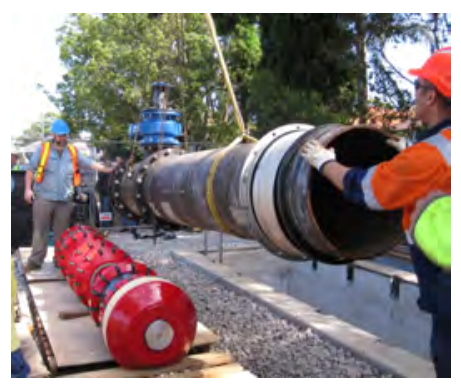

(b) ILI tool.

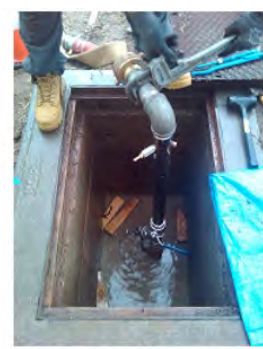

Transient Pressure Wave Generator

Figure 1: Example of various configurations of NDT tools.

NDT techniques produce results that tend to be a trade-off between deployment costs and information gain. Local inspection techniques (i.e. 1 to 3 meters) can provide dense measurements but are time-consuming and generally costly per unit-length as significant preparatory civil works are required (excavations, network re-routing for guaranteed supply, traffic control, etc). Moreover inspections can only be undertaken at locations which are accesible from the surface. An example of these tools can be seen in Fig. 1a.

On the other hand, the taxonomy of long-coverage tools can be broadly split into techniques that provide average pipe wall measurements over longer distances (generally from a few to 100s of meters, even kilometers), and in-line intrusive (ILI) devices ("smart pigs") deployed inside the pipeline to inspect in higher detail over longer distances (generally 100's of meters to kilometers to make it more cost-effective), while propelled by the operating pressure of the fluid.

The former are generally deployed by accessing the external pipe wall or water column at a few access points spread over the length of the pipeline, either through small key-hole excavations or through external access points such as valves or hydrants. As such they tend to have low or no impact in the continuing operation of the pipeline and are more affordable alternatives for condition assessment. An example can be seen in Fig. 1c. However given the averaging nature of their results, these tools are aimed at providing an initial screening of the condition of an asset, and lack the ability to provide the type of detailed geometry information needed to ascertain likelihood of pipe failure.

Flow-driven ILI tools, on the other hand, are inserted into the charged water column either through standard large appurtenances present in critical mains, or more often than not via dedicated launch and retrieval mechanisms, as depicted in Fig. 1b. While these tools are able to provide direct measurements related to the pipe wall condition over 
long distances, they do so at the expense of higher disruption to the utilities and combined costs from the substantial civil engineering support from the utility prior, during and post inspection. Moreover, the effectiveness of these techniques has not been fully established within the industry given the consequential validation investment required to do so in a statistical meaningful way.

ILI tools present additional shortcomings in the pursuit of attaining an accurate depictions of the condition of a pipe wall:

- they are at the mercy of the pressure of the fluid driving them (both in the tethered and free-flowing case).

- should the tools be operated in de-watered conditions, they necessitate complicated winch mechanisms between entry and exit points.

- operating parameters need to be closely controlled (e.g. tool velocity), meaning that discriminating flow controls need to be in place, not necessarily an easy feat to achieve in a complex interconnected network.

- they lack the ability to do fine control and adjustments for mapping (e.g. ensuring tight tolerances in sensor lift-off, repeatability, rectify missed measurements).

Driven by the needs of the water industry the work hereby presented describes the development and field testing of a novel internal NDT inspection robotic vehicle able to:

1. undertake localised, controlled inspections.

2. generate dense NDT mapping suitable for condition assessment and failure prediction.

3. tightly control inherent lift-off during sensing (as induced by the presence of non-magnetic cement lining and pipeline wall irregularities)

4. access arbitrary (within tether range) pipeline spools from a single point of entry, hence reducing costs to utilities and allowing inspection of inaccessible sections from the surface (e.g. under a rail pass) and minimising disruption to customer (e.g. a pipeline under a driveway).

While the proposed solution requires pipes to be de-watered for deployment, this serves a clear mandate from the utility sector that necessitates a robotic NDT inspection vehicle that can be deployed in an opportunistic manner to ascertain the condition of a particular pipeline, specifically when a mains break occurs, or on the back of a valve inspection or repair program when pipelines are inevitably taken off-line. Moreover, while time is always at essence in any maintenance and inspection routines, this is particularly the case for critical assets that need to be put back on-line as soon as feasibly possible. To that end, an efficient robotic inspection solution with the ability to produce detailed dense maps fitting for pipe failure analysis from limited sets of inspection data is highly desirable as that would minimise both collection time and information losses against the original wall thickness maps. The use of Gaussian Processes is thus proposed in this work to model the spatial dependencies present in the interspersed thickness data collected, and to recover form these the required detailed pipe wall geometries.

The remainder of this paper describes such an NDT robot for the inspection of buried network infrastructure and the novelties behind its inception. The manuscript is organized in two main parts. The first part covers the hardware design which includes Section 2, where the sensing capabilities used to measure the pipeline wall thickness are first established, and Section 3 where the locomotion choices and controls for the proposed robotic platform given the specifics of a pipeline environment are then described. The second part describes the Gaussian Process model used to generate maps of wall thickness based on the measurements taken by the robotic platform, described in Section 4, linked to the actual detailed inspection results from deployment in the network of a water utility given in Section 5 . Learnings from the development of the robot and the field deployment have been discussed in Section 6 in the hope that those insights may aid interested readers pursuing related ouctomes, whilst concluding remarks and further work are then collected in Section 7. 


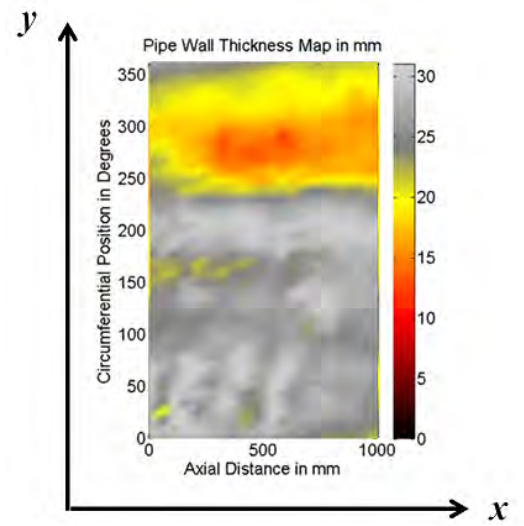

(a) Axis of 2.5D thickness map.

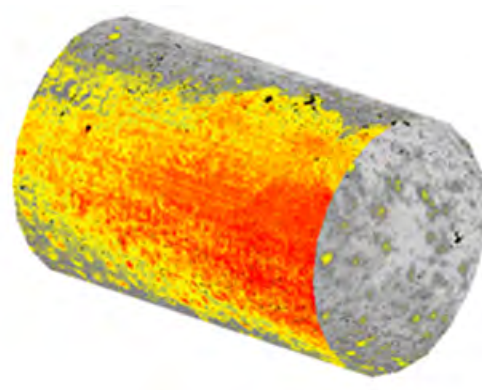

(b) Rolled pipe thickness map.

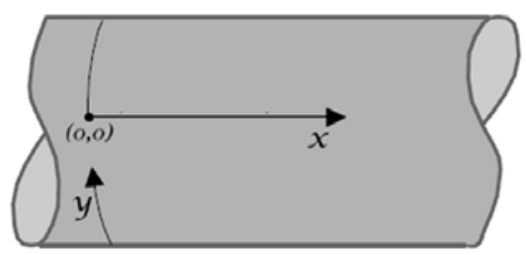

(c) Axial $x$ and circumferential $y$ coordinates aligned on a pipe.

Figure 2: Axial and circumferential coordinates of a 2.5D pipe thickness map.

\section{NDT Pipeline Wall Inspection}

Recent research in the space of stress analysis and failure prediction of critical CI water mains has revealed that over and above pit depths, as traditionally provided during condition assessment of a critical asset, there is a need to ascertain the presence and geometries of large corrosion patches in the pipe walls (Ji et al., 2017; Kodikara et al., 2016), such as those depicted in Fig 16d. There exist a wide range of NDT technologies developed for the purpose of material characterisation for CI (Liu and Kleiner, 2013), yet the provision to build dense 2.5D maps of remaining wall geometries for lined water mains has driven the need to design an internal inspection tool around Pulsed Eddy Current (PEC) sensing technology, as a proven technique typically used in the NDT sector for ferromagnetic material thickness estimation (Huang et al., 2010; Xu et al., 2012; Huang et al., 2011), resilient to sensor lift-off. It is noteworthy to emphasise that while there are a myriad of commercial NDT tools available, they are mostly aimed at visual inspection of an asset, or target carbon steel with the ultimate aim to poinpoint single pitting deficiencies reliably. To date, the authors are not aware of any NDT tools able to densely map arbitrary spools of thick CI material, as it is the intention in this work.

Fig. 2 enables interpreting a typical 2.5D maps of remaining wall thickness as depicted throughout this article, and the conventions shown hold for all thickness maps presented herein. The axial location indicates the distance along the pipe's longitudinal axis, while the circumferential location represents rotational degrees around the pipeline. $0^{\circ}$ and $360^{\circ}$ coincide on the top (crown) of the pipe, denoting the direction pointing vertically upwards perpendicular to the pipe's cylinder axis. As shown in Fig. 2c, the direction of Y-axis increment is counter-clockwise when looking towards the positing $x$ direction, or the direction of robot's travel. It should be noted that despite the visual representation of the circumferential dimensions in degress throughout the paper to aid the reader's intuitive understanding of cilyndrical measurements around a pipeline, in mapping terms they are treated as 2D length measurements in $\mathrm{mm}$ in the Euclidean space, for which a single thickness measurement is obtained via the PEC sensor (hence $2.5 \mathrm{D}$ maps). The colour bar to the right of the thickness maps is a legend representing thickness in $\mathrm{mm}$, between black ( $0 \mathrm{~mm}$, or a through-hole) and light grey $(30 \mathrm{~mm})$. In the field inspection results presented in Section 5, longitudinal locations are in reference to the origin set at the robot's entry point to the pipe.

\subsection{Developed PEC Sensing System}

A typical PEC sensing system developed for ferromagnetic materials consist of an exciter coil, a detector coil, a voltage pulse generator for excitation and an amplifier for the detected signal. A block diagram of the PEC sensing set up developed for this work is shown in Fig. 3a. Given the size of the pipes of interest the footprint of the sensor used was $50 \mathrm{~mm}$, indicating that it measures the average thickness of a $50 \mathrm{~mm} \times 50 \mathrm{~mm}$ area under the sensor. An 


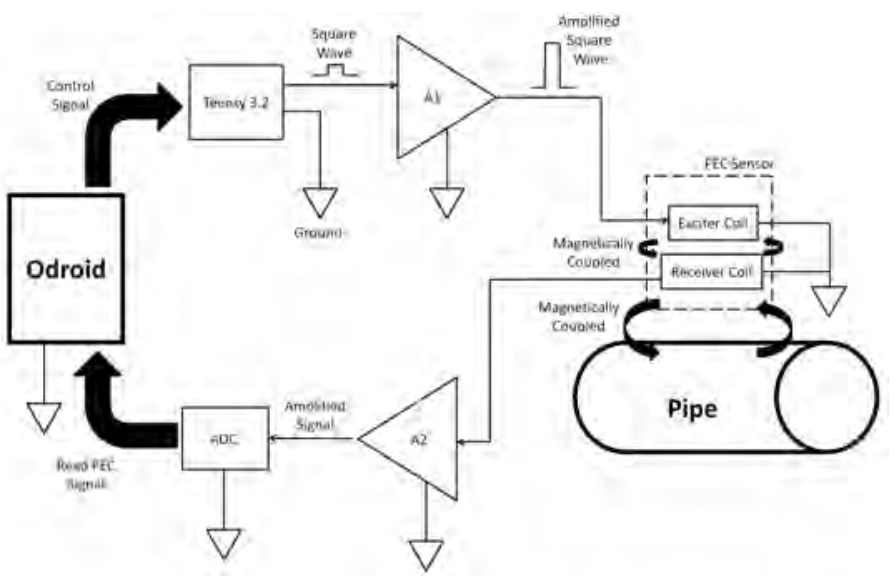

(a) PEC sensing operating diagram.

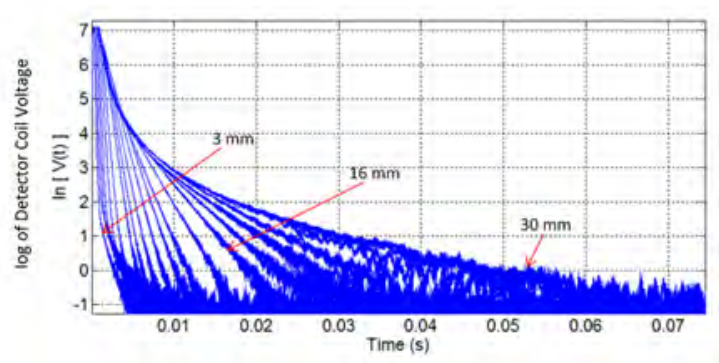

(b) Typical PEC signals on CI thicknesses.

Figure 3: PEC sensing setup embedded in the inspection robot (left), and typical PEC signals.

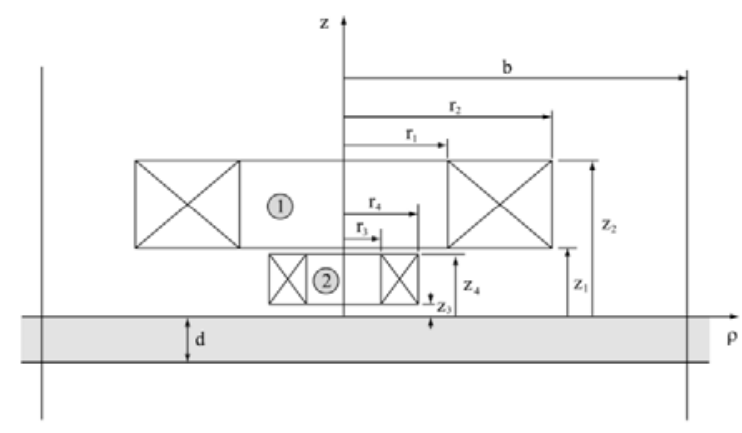

(a) Cross section of a typical coil based circular PEC sensor (adapted from (Fu and Bowler, 2006)).

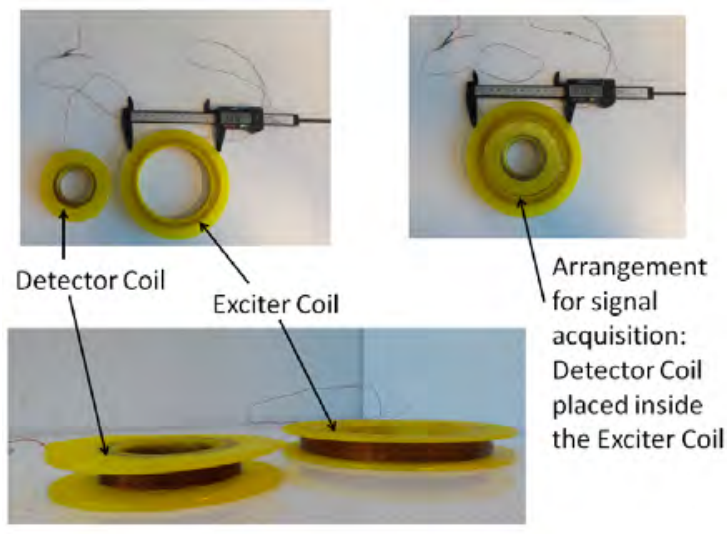

(b) PEC sensor for cast iron pipe assessment (Ulapane et al., 2017).

Figure 4: Typical coil based circular PEC sensor architecture.

example of a typical PEC sensorcoil arrangement is illustrated in Fig. 4. Signals captured from the system on different CI thicknesses are shown in Fig. $3 \mathrm{~b}$ and as reported in the literature features can be extracted from such signals which can be directly linked to material thickness (Huang and Wu, 2015; Ulapane et al., 2014; Ulapane et al., 2017).

\subsection{Validation of PEC Robot Sensor Setup}

The validity of the sensor arrangement was first assessed by comparing results on the exhumed CI pipe in Fig. 5 with intact cement lining. The objective was to evaluate how well the measurements agree if a section of the pipe is scanned externally and internally via cement lining. External measurements were performed on known locations with the aid of the grid pattern marked in Fig. 5a. The same locations were scanned internally as shown in Fig. 5b with the aid of the robot localized with reference to the pipe's edge. Measurements were recorded by placing the sensor centred on the grid squares at $50 \mathrm{~mm}$ distance increments along rings in the circumferential direction, whilst distance between consecutive rings was set to $100 \mathrm{~mm}$ to speed-up the inspection process, since thus generated thickness maps can be then upsampled with minimal information loss as will be shown in Fig. 16d. The rationale and methodology for this will be further elaborated on in the following two Sections. Strong agreement between both measurements was notable as depicted in Fig. 6; the error histogram in Fig. 6e, calculated by subtracting internal thickness estimates from 


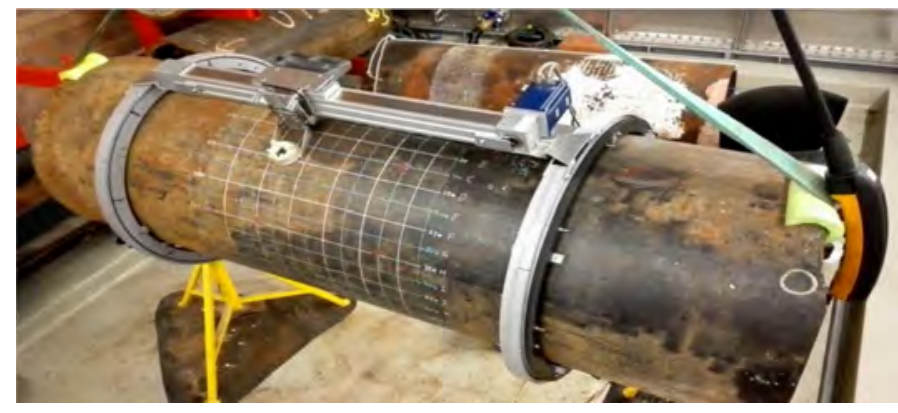

(a) Exhumed pipe on which internal and external measurements were performed.

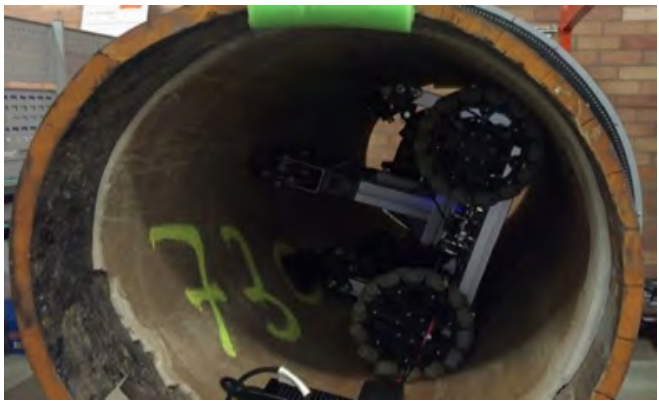

(b) Pipe assessment robot performing internal measurements.

Figure 5: Laboratory setup with exhumed pipe for internal and external PEC validation.

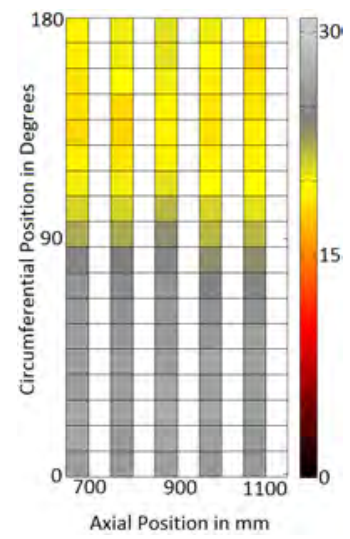

(a) External.

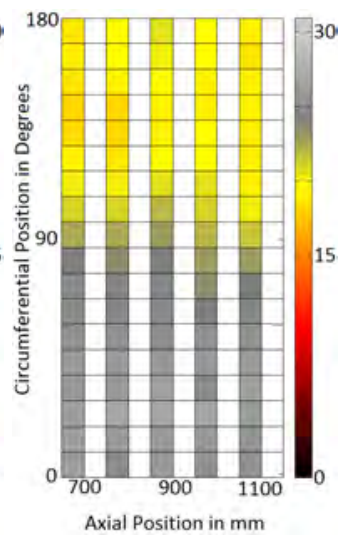

(b) Internal.

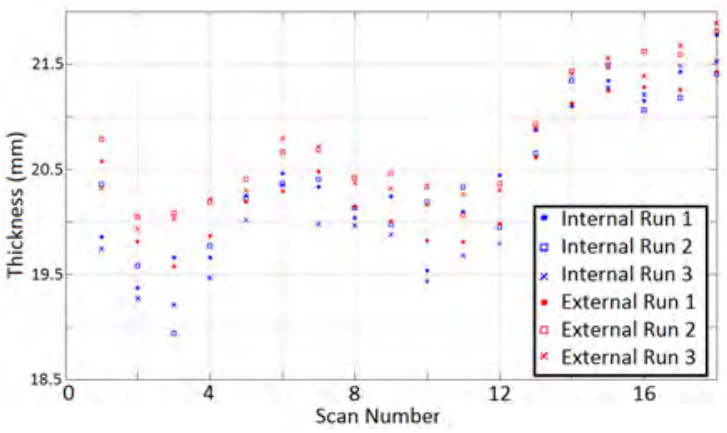

(c) Repeatability of straight line test.

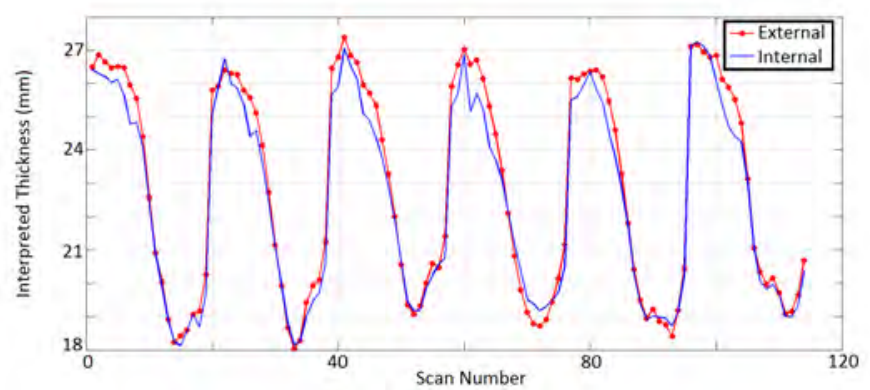

(d) One-on-one comparison 6a vs. 6 b.

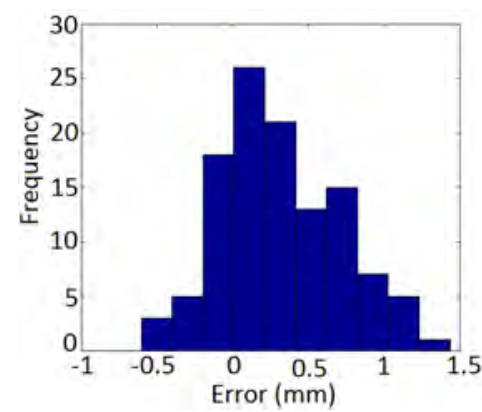

(e) Error histogram 6a vs. 6b.

Figure 6: Thickness maps obtained from internal (in blue) and external (in red) PEC sensor measurements to contrast the validity of an internal sensor deployment mounted on the robotic device when operating through the internal layer of cement lining. Two examples are provided, a rectangular section $\left(500 \mathrm{~mm}\right.$ longitudinally, and $180^{\circ}$ circumferentially) - collected in $6 \mathrm{a}, 6 \mathrm{~b}, 6 \mathrm{~d}$ and $6 \mathrm{e}$, and an internal versus external repeatability straight line test shown in $6 \mathrm{c}$.

external ones hints at a small positive bias in the error, with a mean and standard deviation of $0.323 \mathrm{~mm}$ and $0.417 \mathrm{~mm}$ respectively. This is an expected result since marginally better sensitivity can be expected when scanning externally (particularly for higher thickness), as the sensor touching the pipe wall can achieving stronger penetration than from the inside given the lift-off effect induced form the cement lining layer. The errors are indicative of acceptable agreement between internal and external measurements confirming the sensor's suitability for internal assessment of pipes via cement lining. In another sensor verification experiment, Fig. $6 c$ shows results of a repeatability test carried by 


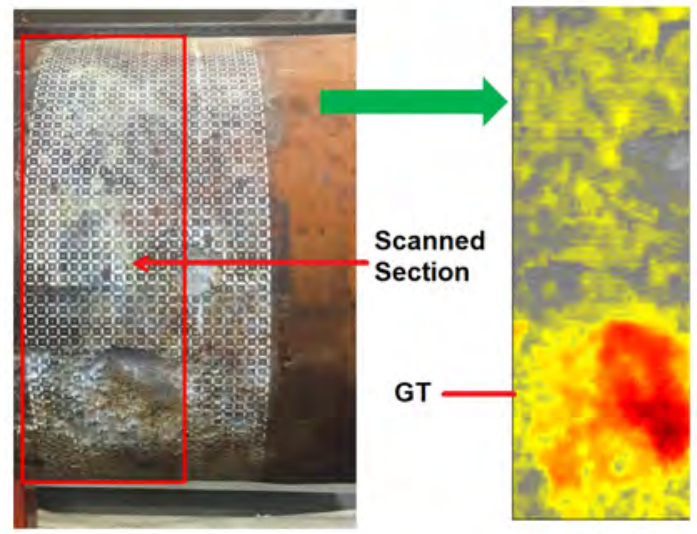

(a) Pipe section and high resolution GT map.

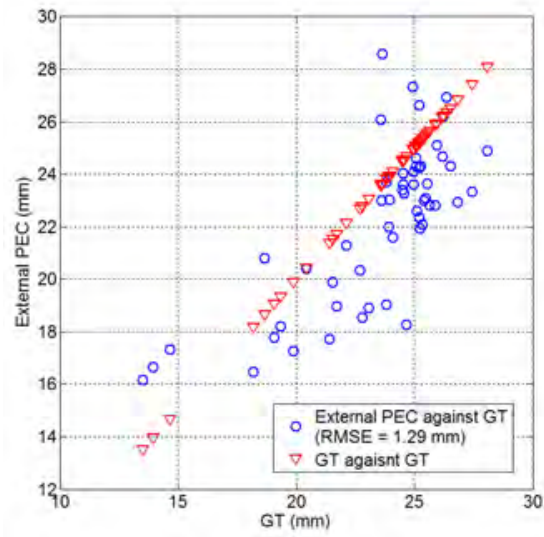

(b) Estimates against laser GT.

Figure 7: External PEC thickness estimates against laser groud truth (GT).

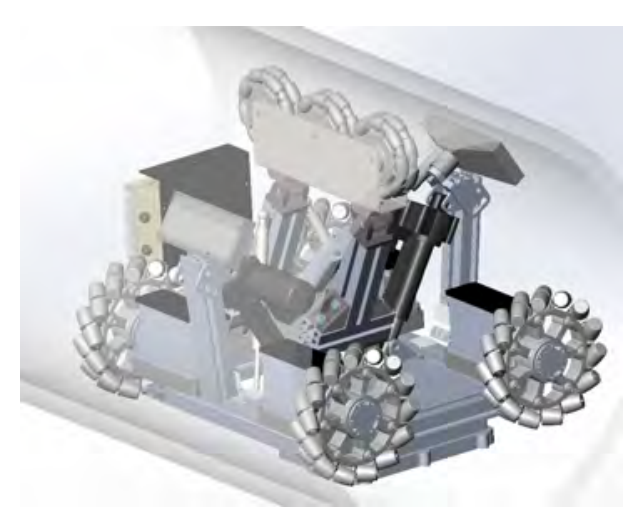

(a) Solid model design.

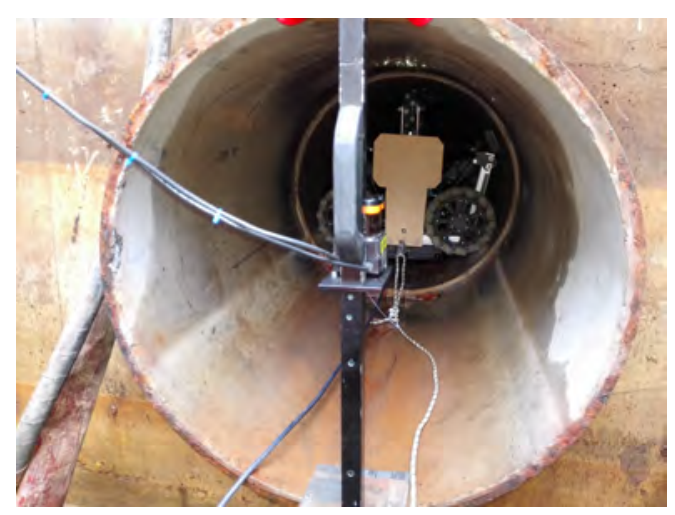

(b) Robot inspecting a pipeline.

Figure 8: NDT inspection robot design, and during field deployment in a pipeline.

measuring a straight line along the pipe six times (three times internally and three times externally, with each line having 18 measurements); the average variation on a location was less than $1 \mathrm{~mm}$, indicating appreciable measurement repeatability.

Further to the comparison of internal versus external deployment, the PEC sensor arrangement was also validated on a pipe whose actual wall thickness ground truth (GT) had been previously obtained, with the results collected in Fig. 7. Attaining the GT is a destructive process, whereby the pipes are first exhumed, then both internal and external pipe surfaces are grit-blasted to remove rust and graphitization, the by-products of the corrosion process inflicted on a buried pipeline, leaving only the bare metal - the target of the PEC sensor measurement. Both surfaces are then reconstructed with a high-resolution 3D laser scanner and ray-tracing performed on the collocated upsampled internal and external pipe surface point clouds to derive the GT thickness maps at a resolution of $0.6 \mathrm{~mm}$ (Skinner et al., 2014). This high resolution GT can then be downsampled to the sensor's $50 \mathrm{~mm}$ footprint by means of averaging so as to match the PEC sensor measurements in order to provide meaningful comparisons. A Root Mean Square Error (RMSE) of $1.29 \mathrm{~mm}$ was observed between external PEC measurements and GT, indicating reasonable agreement even when challenged by significant defects as evident from the testing pipe depicted in Fig. 7a, selected to better capture variability in the remaining wall thickness. 

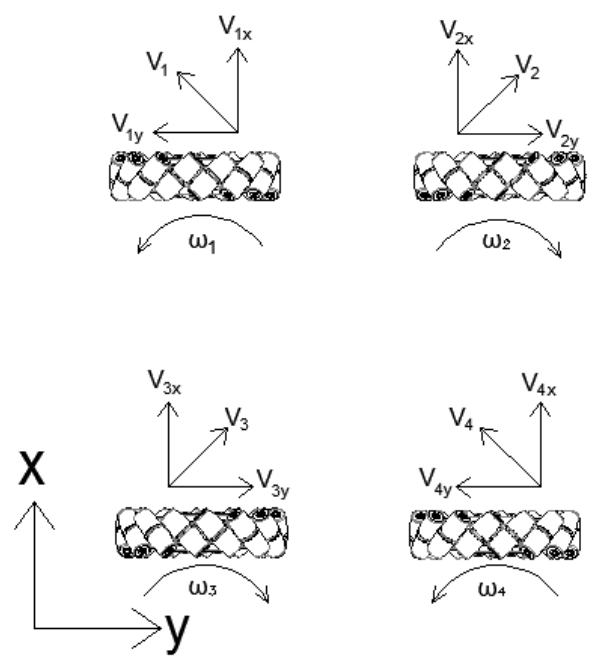

(a) Top view.

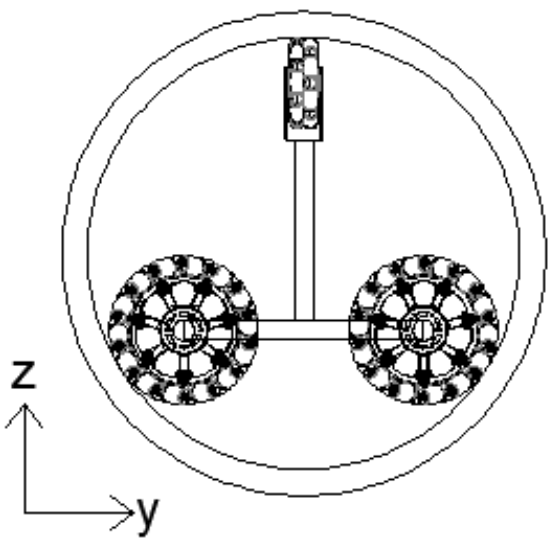

(b) Front view.

Figure 9: Adopted self-alignment mecanum wheel layout. Longitudinal wheel spacing along the $\mathrm{x}$-axis prevents angular motion about the $\mathrm{z}$-axis, therefore maintaining a heading along the longitudinal pipeline direction. The pipe wall stops the robot translating horizontally along the y-axis - instead resulting in the full circumferential rotation desired for the pipe inspection task given the additional support of the deployed vertical stability arm as shown in $9 \mathrm{~b}$, which also allows for consistent surface contact of all the mecanum rollers (at the expense of increased friction with the pipe walls).

\section{NDT Robot Kinematics, Locomotion and Control}

The robotic NDT mapping unit was designed to allow accurate positioning of sensors internally on the pipe surface, in a robust and repeatable manner. To achieve this, a mechanism designed to self-align inside the pipe while providing circumferential and longitudinal control with a single actuation to place sensors against the pipe inner wall was developed. The CAD model and a deployment in the field after construction is depicted in Fig. 8.

\subsection{Mechanical Design}

Mecanum wheels were selected for the robot locomotion. In planar applications they enable holonomic robot motion as they allow control in all three degrees-of-freedom (DoF) available to the robot (Xie et al., 2015). For this application it is only necessary to control two degrees-of-freedom, longitudinal and circumferential motion. By applying a nonstandard wheel configuration it is possible to exploit the unique geometry of the operating environment to passively align with the central pipe axis, automatically tracking the pipe should minor changes in direction occur. Fig. 9a and Fig. $9 \mathrm{~b}$ demonstrate the layout designed to achieve these requirements. In this configuration, the axis of rotation of the pipe contacting rollers all pass through a single point allowing the robot to rotate freely about this point in response to an external force. When resting on a cylindrical surface, such as a pipe wall, an external restoring force is generated in response to angular disturbances which acts to return the robot to the aligned position.

Control in the longitudinal direction and rotation about the circumferential direction are achieved by controlling wheel velocities using the kinematic relations derived in Eq. 1, which follow standard forwards kinematic equations in simplified form (Taheri et al., 2015), where $v_{\mathrm{x}}(t)$ reflects the longitudinal velocity $(\mathrm{m} / \mathrm{s}), v_{\mathrm{y}}(t)$ is the circumferential velocity $(\mathrm{m} / \mathrm{s}), \omega_{i}(i=1 \ldots 4)$ is the wheel rotation speed $(\mathrm{rad} / \mathrm{s}), \omega_{\mathrm{z}}(t)$ denotes angular velocity on the x/y plane, $r$ is the wheel radius $(\mathrm{m})$ and $l_{\mathrm{x}}, l_{\mathrm{y}}$ indicate the wheel separation and body length respectively. 


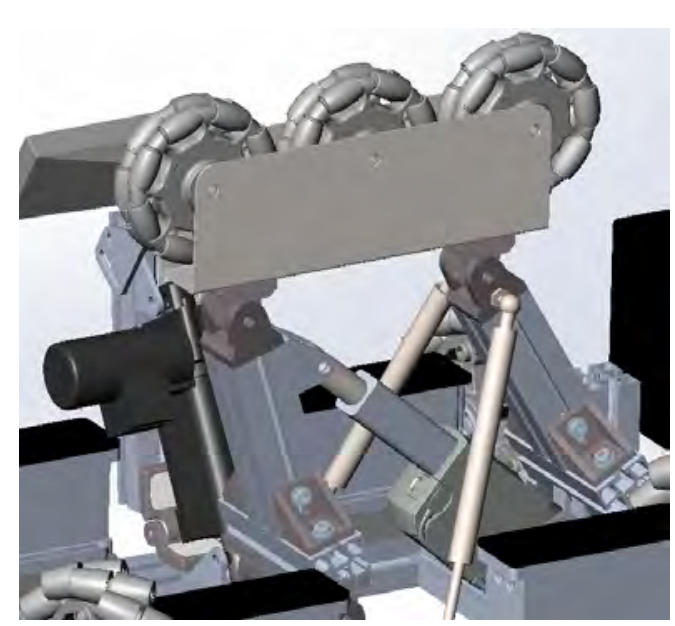

(a) Arm design.

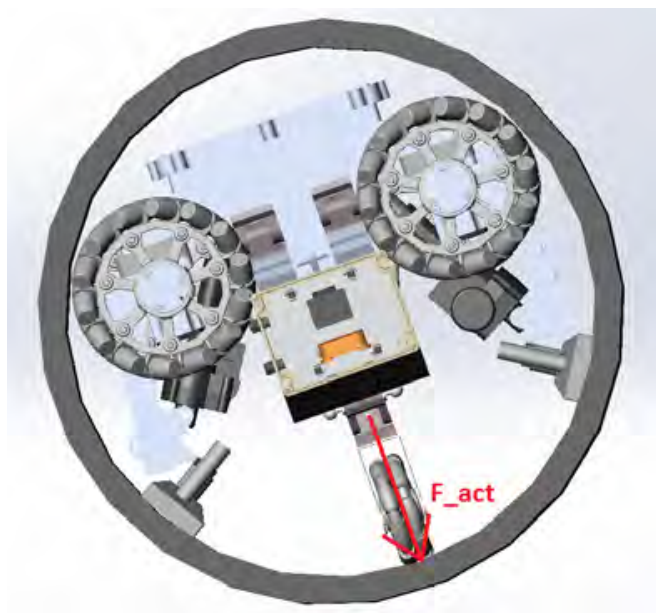

(b) Rotated stabilising arm.

Figure 10: Stabilising arm.

$$
\begin{array}{r}
v_{\mathrm{x}}(t)=\left(\omega_{1}+\omega_{2}+\omega_{3}+\omega_{4}\right) \times \frac{r}{4} \\
v_{\mathrm{y}}(t)=\left(-\omega_{1}+\omega_{2}+\omega_{3}-\omega_{4}\right) \times \frac{r}{4} \\
\omega_{\mathrm{z}}(t)=\left(-\omega_{1}+\omega_{2}-\omega_{3}+\omega_{4}\right) \times \frac{r}{4 \times\left(l_{\mathrm{x}}+l_{\mathrm{y}}\right)}
\end{array}
$$

It is evident from Eq. (1) that maintaining zero angular velocity (and therefore a constant heading) is a wheel speed control task. As demonstrated in Fig. 9b, wheel spacing prevents angular motion about the $\mathrm{z}$ axis, therefore maintaining a heading along the longitudinal direction. The pipe wall stops the robot translating horizontally - instead resulting in the circumferential rotation that is desired for this task. It is however essential that the angular velocities of diagonally opposite wheels are matched to prevent excessive motor loads precisley given the robot is constrained in the $\mathrm{z}$ axis. Driving each pair of diagonally opposite wheels with a single motor would achieve this requirement, however, the required drivetrain is complex and in the proposed designed control of each separate motors is implemented in software, as discussed below as part of the system overview.

To maintain stability during circumferential rotations, a set of free-wheeling omni-wheels are mounted on a parallel four bar linkage shown in Fig. 10a. This is linked to a pair of gas struts to provide a consistent opposing force to the mecanum wheels surface contact point. The applied force cancels out the gravity vector as the robot rotates, allowing consistent continuous surface contact for each of the wheels rollers. To achieve this the assembly is pressed against the pipe wall with a preload of approximately twice the robot weight, maintaining control authority regardless of orientation while simultaneously compensating for variation in pipe diameter. A linear actuator is included to retract the omni-wheels from the pipe surface during insertion. This actuator features a spline so that it does not affect the self correcting behaviour of the parallel linkage during normal operation. Fig. 10b demonstrates the applied force during the robots rotation along the vector $F_{-}$act.

The PEC sensors are coupled to actuated lever arms using a stiff rubber joint. This joint allows the sensor to conform to the pipe surface in the presence of minor irregularities while maintaining a precise placement. The actuators drive until a stall condition is detected, allowing the sensor to be reliably placed on the pipe surface regardless of pipe variations or actuator drift.

Fig. 11 depicts the final robot in more detail in a pipeline in the laboratory (left), and in an open-cut pipeline for easier viewing in the field. 


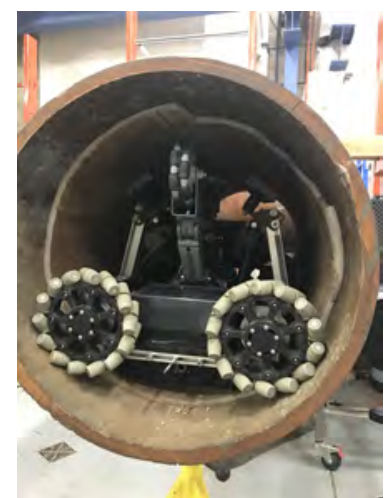

(a) NDT inspection robot front view.

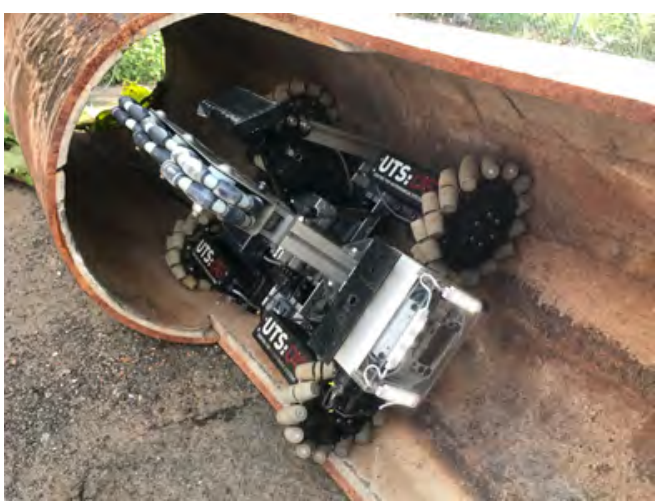

(b) NDT inspection robot in an open-cut pipe.

Figure 11: The NDT inspection robot (right figure depitcs the configuration with only one sensing arm mounted on).

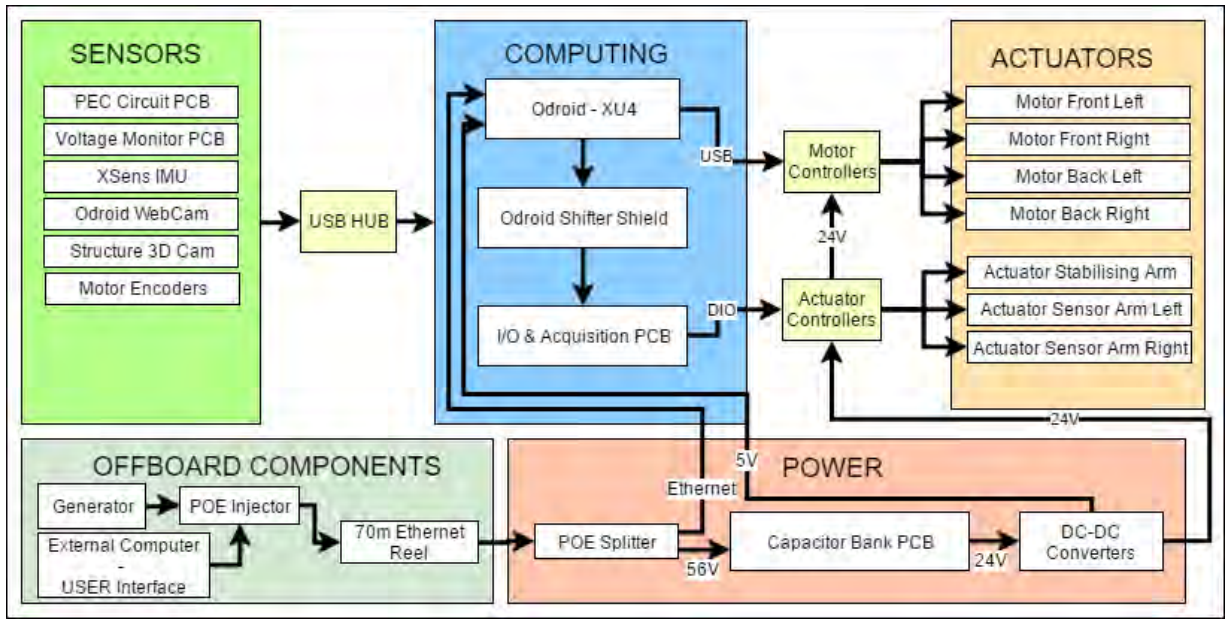

Figure 12: System block diagram depicting the four on-board major components on the robotic system (power, sensors, computing and actuators), and the off-board components linking the robot to a user-driven computer via a $70 \mathrm{~m}$ powerover-ethernet tether.

\subsection{System Overview}

The system uses two computers, one on-board the robot for data acquisition and actuator control, and one outside the pipe for the user interface. The entire system runs from a generator on the surface with power delivered to the robot with a power over ethernet $(\mathrm{PoE})$ connection. The user interface can receive data and issue control commands back to the operator in real-time over the local area network (LAN) connection provided by the same ethernet tether. Fig. 12 demonstrates the overall hardware system layout whilst Table 1 list details about each component.

The long deployment duration precludes battery operation hence the choice of tethered PoE to provides for significantly longer operation times. The POE injector provides $60 \mathrm{~W}$ of power, the maximum supplied by readily available off-the-shelf equipment, so an ultracapacitor bank and bespoke charger was developed to supply bursts of high power while ensuring that the PoE equipment maintains an optimal power delivery rate. While deploying the sensors and taking a reading the average system power is $40 \mathrm{~W}$. This increases dramatically to $100 \mathrm{~W}$ when the motors are driven to reposition the robot. Since the time spent driving the motors is relatively low in comparison to sensing acquisition the overall average power requirement is less than the $60 \mathrm{w}$ supplied by the PoE system. Thus, the chosen setup provides a steady power supply for the overall system on the condition that high power maneuvers are not sustained for extended periods, as is the case for the inspection of critical water mains which lay flat and straight in the ground. 
Table 1: Core Component specifications.

\section{COMPUTING SENSORS}

CONTROL
Odroid XU4 - Arm based single board computer

Xsens Mti-10 IMU w/ Gyro $450^{\circ} / \mathrm{s}$, acc $50 \mathrm{~m} / \mathrm{s}^{2}$

Odroid USB-Cam 30FPS, FOV: $68^{\circ}$

3D Structure Sensor w/ HFOV: $58^{\circ}$, VFOV: $45^{\circ}$

Maxon Motor DCX26L, gear ratio 231:1, sensor 500 counts/turn

Sensor Actuator - linear actuator, max force: $1000 \mathrm{~N}$

Stability Actuator - linear actuator, max force: $2500 \mathrm{~N}$

PoE injector/splitter, 60W, 70m cable reel

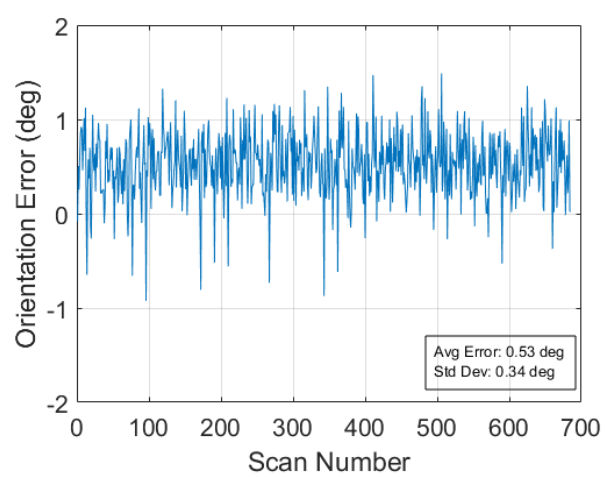

(a) Circumferential repeatability.

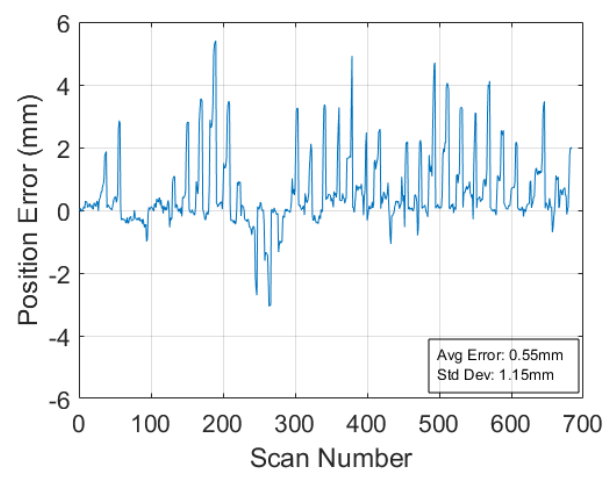

(c) Longitudinal error.

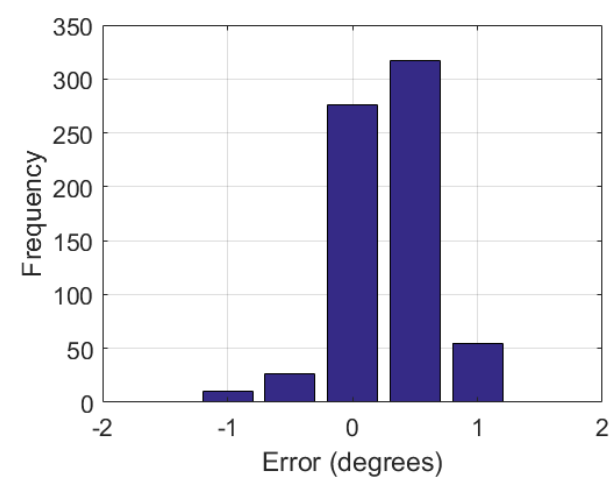

(b) Circumferential error histogram.

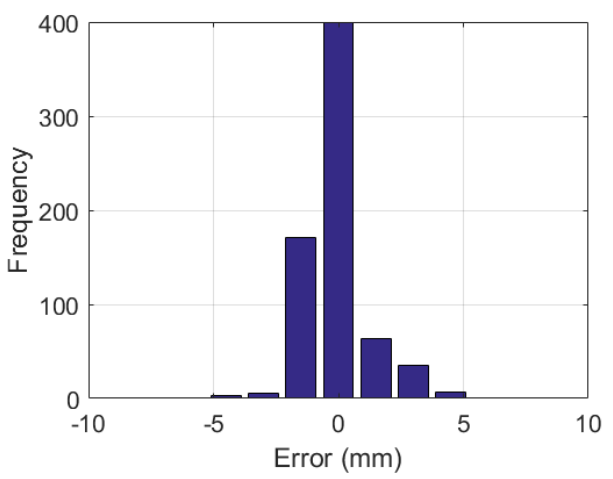

(d) Longitudinal error histogram.

Figure 13: Circumferential and longitudinal consistency. Circumferential deviations (top graphs) represent orientation errors of repeated commanded $10^{\circ}$ increment motions, as measured by an on-board IMU, whilst longitudinal errors shows the axial drift during these motions as measured by an external LIDAR (see Fig. 8b)

The on-board Odroid, running Linux and the Robotic Operating System (ROS), receives data from the sensor suite through a powered USB hub and controls on-board actuators via digital input/outputs pins. Each sensor has it's own monitoring node to manage incoming data and publish to the communication layer. Custom task allocation/behaviour nodes then subscribe to the data streams, processing and publishing control commands as required to the motor and actuator nodes. System control is accomplished using a state machine, which allows both user and autonomous control modes for consistent data retrieval and safe user override. When switched into automatic scanning mode, the circumferential angle and longitudinal position are managed using independent set-point control loops. This simplifies both the kinematics and the algorithms required for control. Controlling circumferential angle is achieved using the on-board IMU and a standard PID control algorithm. The IMU publishes attitude data to ROS at a fixed rate of $100 \mathrm{~Hz}$. 
As each data packet is received, the attitude data is transformed into the local coordinate frame to maintain consistency even when the pipe is not levelled. Similarly, longitudinal control is achieved using odometry calculated using encoder readings published at a $100 \mathrm{~Hz}$ and filtered to detect wheel stalls. In addition, an overriding human in the loop (HITL) input allows direct control of the longitudinal position. This is used to recover when odometry fails due to motor stalls or excessive wheel slip during the ring-to-ring transitions. A laser distance sensor is utilised to confirm longitudinal position when conditionas are safe to deploy in the excavation pits.

\subsection{Motion Validation}

The circumferential and longitudinal consistency during automated scanning was verified at onsite trials, with angular repeatability and translational slip during rotation being the key metrics providing confidence in the sensor placement accuracy. Data was collected from an IMU to validate robot orientation, and a laser distance sensor was used to confirm longitudinal positions. Fig. 13a shows the measured rotation angle error during repeated scanning cycles of $180^{\circ}$ using $10^{\circ}$ increments. The average offset error of $0.53^{\circ}$ in relation to the set-point was found to be originated by the pressing action of the sensor against the pipe wall. Fig 13c shows longitudinal drift during these scans. An average error of $0.55 \mathrm{~mm}$ was produced with the maximum recorded error being $5.4 \mathrm{~mm}$. Since the PEC sensing method produces a result averaged over a $50 \times 50 \mathrm{~mm}$ area and the scan rings are spaced at increments of $100 \mathrm{~mm}$, a $5 \mathrm{~mm}$ drift in the longitudinal direction is deemed an acceptable inaccuracy well within the safety margins generally assumed for failure prediction analysis in civil infrastructures.

Given the overall sensing and mechanical constraints the system operates at slow speeds: runtime metrics measured during these tests showed an average spool completion time of around 166 minutes, or $1.3 \mathrm{~m} / \mathrm{h}$. This includes 216 seconds of automated scanning for each ring, with 30 to 60 seconds dedicated to motion from one ring to the next.

\section{Gaussian Processes for Spatial Regression Mapping from Sampled NDT Inspection Data}

As mentioned in Section 1 the salient novelty of the proposed robotic integrity assessment is the ability to carry out internal detailed inspections that enable dense mapping where identification of the geometry of wall loss patches can be confirmed. To achive this outcome various inspection patterns were studied to mitigate the slow robot examination speed reported in Section 3. A number of sampling strategies were experimented on and were extensively evaluated through cross validation using twelve thickness maps collected from exhumed pipelines during field trials and groundtruthed as decribed earlier in Section 2.2. This was carried out to determine the best inspection strategy that would minimise information losses against the original $2.5 \mathrm{D}$ wall thickness maps, given the nature of the thickness map data collected (Shi and Valls Miro, 2017). In this Section the proposed strategy undertaken to regress the missing data with the aid of a tailored Gaussian Process is presented.

Modeling spatial dependencies for 2.5D data has been largely studied in the past. Mathematically the problem can be described as a random field which is a collection of random variables of the form $\left\{y_{\mathbf{x}}, \mathbf{x} \in \mathbf{R}^{d}\right\}$, where $y_{\mathbf{x}}$ is the quantity measured at the position $\mathbf{x}$ (Lord et al., 2014). Random fields are also known as spatial processes, for instance, univariate (Kroese and Botev, 2013) or multivariate (Schlather et al., 2015) processes, that are defined for modeling spatially arranged measurements and patterns. Random fields can be statistically specified by mean and covariance (Lord et al., 2014; Kroese and Botev, 2013). When the mean is a constant, depending on the covariances there are stationary random fields whose covariances are invariant under translations, isotropic stationary random fields whose covariances are invariant under both translations and rotations, and anisotropic stationary random fields whose covariances are directionally dependent (Lord et al., 2014; Kroese and Botev, 2013). A more specific type of random field being studied extensively is Gaussian Random Fields (Davies and Bryant, 2013), which is also known as Gaussian Spatial Processes (Kroese and Botev, 2013) or Gaussian Processs (GPs) (Bishop, 2006; Rasmussen and Williams, 2006).

In robotics, GPs have been employed in terrain and surface modeling (O'Callaghan and Ramos, 2012; Smith et al., 
2010; Vasudevan et al., 2009). In all these works, the utilization varies with the properties of the applications, the structure of the model and the usage of the correlation information. The use of these probabilistic tools for thickness mapping and fusion has recently been tackled by the authors for an application on pipeline condition assessment (Sun et al., 2015; Vidal-Calleja et al., 2014).

\subsection{Gaussian Processes}

GPs define the probability distribution over functions, any finite number of which have consistent joint Gaussian distribution. Consider $n$ thickness-location pairs $\mathscr{D}$ defined as:

$$
\mathscr{D}=\left\{\left(y_{1}, \mathbf{x}_{1}\right),\left(y_{2}, \mathbf{x}_{2}\right), \ldots,\left(y_{n}, \mathbf{x}_{n}\right)\right\}
$$

where $\mathbf{x}_{i} \in X$ is the position in $\mathbf{R}^{d}$ ( $d=2$ in the case of $2.5 \mathrm{D}$ data) where the thickness measurements $y_{i} \in Y$ was taken. The data set $\mathscr{D}$ is assumed to be drawn from a noisy process

$$
y_{i}=f\left(\mathbf{x}_{i}\right)+\varepsilon_{i}, \text { where } \varepsilon_{i} \sim \mathscr{N}\left(0, \sigma_{n}^{2}\right),
$$

where noise $\varepsilon_{i}$ follows independent, identically distributed zero-mean Gaussian with variance $\sigma_{n}^{2}$. GPs are used to learn the distribution $p(f \mid X, \mathscr{D})$ from $\mathscr{D}$ and have the capability of inferring $p\left(f \mid X^{*}, \mathscr{D}\right)$ for arbitrary location $X^{*}$.

Having specified the mean and covariance functions ${ }^{1}$ and identified the hyper-parameter set $\theta$, parameter estimation can be conducted through optimization by maximizing the likelihood function as described in equation 4 .

$$
\begin{array}{r}
\log p(\mathbf{y} \mid X)=-\frac{1}{2}(\mathbf{y}-m(X))^{\top} K_{y}^{-1}(\mathbf{y}-m(X)) \\
-\frac{1}{2} \log \left|K_{y}\right|-\frac{n}{2} \log 2 \pi
\end{array}
$$

where $m$ and $K$ are the mean and covariance functions respectively, and $K_{y}=K(X, X)+\sigma_{n}^{2} I$ denotes the joint prior distribution covariance of the function at positions $X$. The variance of the noise $\sigma_{n}^{2}$ constitutes another parameter to be learned together with $\theta$.

Inference at a finite set of query locations $X^{*}$ can be performed by calculating the predicted mean $\mu_{P}$ and covariance $\Sigma_{P}$ :

$$
\begin{gathered}
\mu_{P}=m\left(X^{*}\right)+K\left(X^{*}, X\right) K_{y}^{-1}(\mathbf{y}-m(X)) \\
\Sigma_{P}=K\left(X^{*}, X^{*}\right)-K\left(X^{*}, X\right) K_{y}^{-1} K\left(X^{*}, X\right)^{\top}
\end{gathered}
$$

The covariance matrix $K\left(X^{*}, X\right)$, obtained from a given covariance function $K$, is indicative of the cross-correlation between the function at $X^{*}$ and the training inputs $X$.

\subsection{Covariance Function Design}

GPs are thus completely specified by the choice of mean and covariance functions. The mean function can be usually set to be a constant value, whilst the covariance function controls the smoothness of the process, and its parameters govern the effective range of correlation and the variability observed in the data. There is no single covariance function that fits all modeling tasks. Depending on the purpose at hand and any insights that might be available from the underlying physical phenomenon described by the data, modified or composite covariance functions may allow more flexibility in the model. Indeed, the usage of prior knowledge in choosing appropriate covariance functions is encouraged in the literature (Tesch et al., 2011), e.g. using periodic covariance functions in the analysis of seasonal variation and physical phenomena (Rasmussen and Williams, 2006) (Tartakovsky and Xiu, 2006).

\footnotetext{
${ }^{1}$ The terms covariance and kernel function are used indistinctively.
} 
In effectively modelling the wall thickness of buried pipelines a number of covariance functions were considered which included characteristics related to the physical properties of the target to be modelled. Three commonly used stationary kernel functions (detailed in the next Section) with additional characteristics revealed by the data in terms of directionality and periodicity were tested. These were incorporated in the form of a 2D anisotropic composite covariance function with a periodical wrapping construction. The period in the circumferential direction was clamped to guarantee the $2 \pi$ periodic property of a pipe wall thickness map. An example with a $2 \mathrm{D}$ Matern kernel $(v=3 / 2)$ is shown in equation 7

$$
K\left(X, X^{*}\right)=K(r)=(1+\sqrt{3} r) \exp (-\sqrt{3} r)
$$

where the input distance $r$ is defined by

$$
r=\frac{1}{l} \sqrt{\left(X-X^{*}\right)^{T}\left(X-X^{*}\right)}
$$

for an isotropic kernel, with $l$ being the length-scale, and by

$$
r=\sqrt{\left(X-X^{*}\right)^{T} \wedge^{-2}\left(X-X^{*}\right)}
$$

for an anisotropic kernel, where $\wedge$ is a diagonal matrix with characteristic length-scales $l_{1}$ and $l_{2}$ on the main diagonal.

In the case of modelling periodical data, an established approach is warping. This is generally done by mapping each one-dimensional input variable $x$ to two-dimensional input variable

$$
\mathbf{u}(x)=\left[\sin \left(x_{p}\right), \cos \left(x_{p}\right)\right]
$$

where $x_{p}=2 \pi x_{p}$, and $p$ is the period parameter, hence constructing a covariance matrix $K\left(\mathbf{u}(X), \mathbf{u}\left(X^{*}\right)\right)$ to turn an anisotropic kernel periodic (Rasmussen and Williams, 2006).

\subsection{Model Selection}

Model selection was carried out by using the ground-truth dataset refered to in Section 4, composed of twelve 3D laser thickness maps attained from exhumed pipeline spools from the same pipeline, so that the original 2.5D wall thickness maps could be recovered via the proposed GP regressor from the in-field sample data collected by the NDT robot.

The proposed covariance function was selected by considering the physical properties of the target to be modelled described in the preceding Section, and by means of comparing the Akaike information criterion (AIC) of each model. AIC is a relative metric between potential models defined as

$$
A I C=2 k-2 \ln (L)
$$

where $k$ is the number of model parameters, and $L$ is the maximum value of the likelihood function for the model in parameter estimation (?). While the Log Marginal Likelihood (LML) is also often used in the literature for this purpose, for models with relatively low complexity - as those proposed in this work - the values of both LML and AIC are comparable, so AIC was selected as arguably it provide a slight advantage over LML in also penalising models with additional complexity for similar fitting, thus discouraging overfitting

Three commonly used kernel functions with different setups were tested to establish the guidance on selecting the most appropriate model. The basic characteristics of the three candidate kernel functions in a simplified isotropic 1D scenario are illustrated in Fig. 14a, given the difficulty in visualising a 2D anisotropic kernel. Table 14b shows that under the same setup, both anisotropic and periodic composite covariance model setups produce lower AIC given the characteristics of the pipeline data. The combination of these two add-ons gives the best performance for 


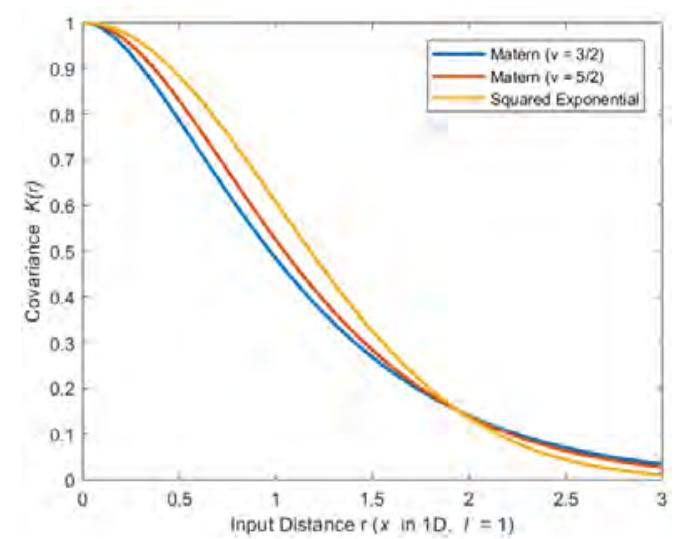

\begin{tabular}{|l|c|c|c|c|c|}
\hline Kernel & $\begin{array}{c}\text { Anisotropic } \\
\text { Add-on }\end{array}$ & $\begin{array}{c}\text { Periodic } \\
\text { Add-on }\end{array}$ & \#Params. & $\begin{array}{c}\text { AIC } \\
(\text { mean })\end{array}$ & $\begin{array}{c}\text { AIC } \\
(\mathrm{std})\end{array}$ \\
\hline Matern $(\mathrm{v}=3 / 2)$ & $\mathrm{N}$ & $\mathrm{N}$ & 4 & 2448 & 1475 \\
\hline Matern $(\mathrm{v}=5 / 2)$ & $\mathrm{N}$ & $\mathrm{N}$ & 4 & 2718 & 1434 \\
\hline Sq. Exp. & $\mathrm{N}$ & $\mathrm{N}$ & 4 & 3531 & 1390 \\
\hline Matern $(\mathrm{v}=3 / 2)$ & $\mathrm{Y}$ & $\mathrm{N}$ & 4 & 2001 & 1411 \\
\hline Matern $(\mathrm{v}=5 / 2)$ & $\mathrm{Y}$ & $\mathrm{N}$ & 4 & 2283 & 1377 \\
\hline Sq. Exp. & $\mathrm{Y}$ & $\mathrm{N}$ & 4 & 3034 & 1415 \\
\hline Matern $(\mathrm{v}=3 / 2)$ & $\mathrm{Y}$ & $\mathrm{Y}$ & 7 & 1922 & 1423 \\
\hline Matern $(\mathrm{v}=5 / 2)$ & $\mathrm{Y}$ & $\mathrm{Y}$ & 7 & 2147 & 1388 \\
\hline Sq. Exp. & $\mathrm{Y}$ & $\mathrm{Y}$ & 7 & 3051 & 1473 \\
\hline
\end{tabular}

(a) Characteristics of the 3 candidate covariance functions (b) Combined kernel models studied with anisotropic and periodic add-ons to a given tested, graphically depicting the statistical relationship be- standalone covariance function, and AIC model selection metric. tween any two points " $r$ " distance appart in 1D.

Figure 14: Gaussian Process kernel model selection. The combined anisotropic and periodic covariance functions were tested on ground truth data thickness maps from a large number of exhumed pipeline spools, and their performance in terms of AIC metric used to select the kernel to regress the data colllected with the NDT inspection robot (low AIC values are indicative of a better fitting).

cylindrical structures such as buried pipes, which corresponds to the observation that pipe wall thickness correlations in the extracted data appear differently in circumferential and axial directions, and the correlation in circumferential direction is $2 \pi$ periodic. Among these kernel functions, Matern v3/2 always produced the lowest AIC under the same setup. Higher order Matern are generally indistinctive from the Square Exponential case, whilst given the apparent trend that less smooth kernels perform better, it would be reasonable to also consider the lowest order Matern v1/2. However, these models become quite "rough" and while functions are continuous they loose the differentiablily, a characteristic that was not readily apparent in the data so was not perceived as a fitting alternative. Therefore, Matern $\mathrm{v} 3 / 2$ with anisotropic and periodic setup was selected as the most appropriate kernel for modeling pipe wall thickness maps.

It is worth noting that reconstruction error was also experimented upon as a model selection metric, and statistical tests (ANOVA) computed on sample mean and variances. It was found that Matern kernels (tested with different hyper-parameters) behaved statistically the same in terms of the reconstruction error. On the other hand, given the dense training data around missing data, Squared Exponential produced statistically significantly worse results than any Matern kernel.

Table 2: Test-bed specifications, adapted from (Valls Miro et al., 2014).

\begin{tabular}{ll}
\hline Year Installed & 1922 \\
Nominal Pipe Diameter & $600 \mathrm{~mm}$ \\
Internal Pipe Diameter & $579 \mathrm{~mm}$ to $590 \mathrm{~mm}$ (with cement lining) \\
External Pipe Diameter & $662 \mathrm{~mm}$ to $666 \mathrm{~mm}$ \\
Nominal Wall Thickness & $27 \mathrm{~mm}$ \\
Material & Pit Cast Iron \\
Internal Liner & Cement (installed in 1964) \\
Cement Lining Thickness & $9.5 \mathrm{~mm}$ to $16.5 \mathrm{~mm}$ \\
Jointing & Lead run joints (with tar soaked hemp \\
& sealants) \\
\hline
\end{tabular}




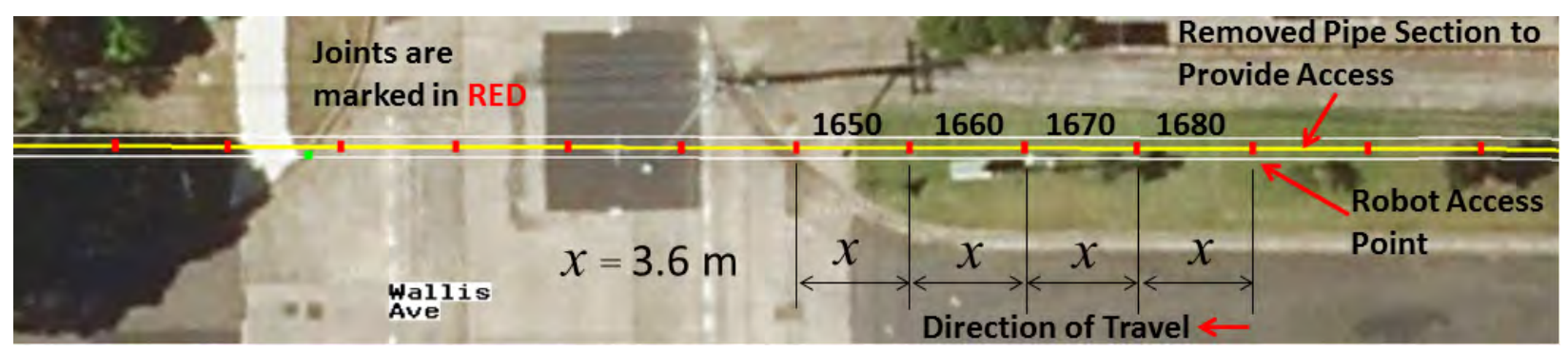

Figure 15: A typical inspection plan supplied to the utility parner for the deployment of the NDT inspection robot.

\subsection{Stability of the Model Selection}

The stability of the AIC model section metric was estimated through bootstrapping (Efron and Tibshirani, 1994) training samples from the twelve GT thickness maps. The theoretical foundation of bootstrap resampling allows estimating the distribution of a statistic using random sampling methods. Specifically in this evaluation, training map samples were randomly drawn with replacement for 100 iterations to feed in AIC parameter estimation and characterize the candidate model. The last two columns in Table 14b collects the results, which indicate that all candidate kernels have similar AIC variances yet the propose anisotropic periodic Matern $(v=3 / 2)$ kernel exibits the lowest AIC mean.

\section{Field Pipeline Inspection Results}

The proposed robotic device has been extensively deployed in a buried $1 \mathrm{~km}$ live CI Cement Lined (CICL) pipeline provided by a utility in Sydney, Australia, in what effectively constitutes a unique worldwide opportunity for the advancement of NDT sensing and automation research in the field (Valls Miro et al., 2014). The pipeline has been decommissioned and is therefore no longer part of the utility's live network. However a connection point to an adjacent $600 \mathrm{~mm}$ water main and various scour valves and hydrants allow for the pipeline to be pressurised and discharged as needed. Details of the pipeline are collected in Table 2. Pipe sections between 3 and $4 \mathrm{~m}$ in length were targetted for scanning by inserting the inspection robot through a removed pipe section, be that a previously replaced section, as shown in Fig. 1b, or a new cut-out. An example of an inspection plan is shown in Fig 15.

Robot localisation with respect to an entry point while travelling towards a section targetted for inspection was done by means of robot odometry, measurement of tether release and accounting for spool joints traversed as seen by the robot camera. Validation from an external laser scanner mounted at the entry point as seen inFig. 8b was also used when it was deemed safe to be deployed in the field excavation pit, and there was line of sight within the laser range. Moreover, discontinuity on spool joints also reveals a characteristic PEC signal comparable to a crack that was also exploited in case of ambiguity about spool length.

After reaching the target spool, circumferential and longitudinal ring inspections were undertaken as described in Section 3 to generate maps such as those depicted in Fig. 16. Following the inspection pattern ascertained in (Shi and Valls Miro, 2017), circumferential rings $100 \mathrm{~mm}$ appart in axial distance were evaluated with the robotic platform, which given the $50 \mathrm{~mm}$ sensor footprint effetively meant skipping every other ring with considerable time savings. Examples of these are shown in Fig 16a, 16b and 16c. Adopting the GP model selection described in Section 4, a model could be learned for each inspected spool and full inference on the missing rings undertaken. An example of the final outcome achieved is shown in Fig. 16d, where measurements indicative of the lead run joints are also shown. Where wall loss is present the spread of the reduction is clearly evident and can be identified and measured. Such patches are modelled as ellipsoids (also depicted in Fig. 16d overlaid over the reconstructed map), and their defining parameters can then be incorporated for stress calculation and remaining life prediction of the asset (Ji et al., 2017).

The final thickness maps gathered by the NDT robot during an extensive period of deployment between 2016 and 2018 


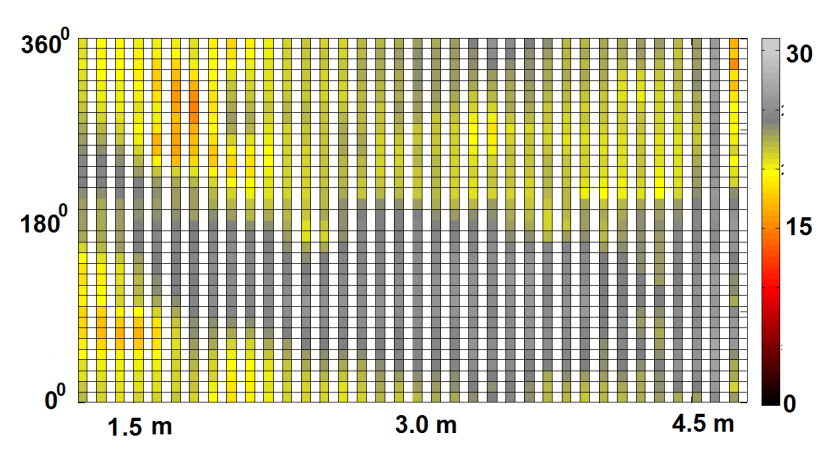

(a) Target 1680 in Fig. 15(plan) and 17b.

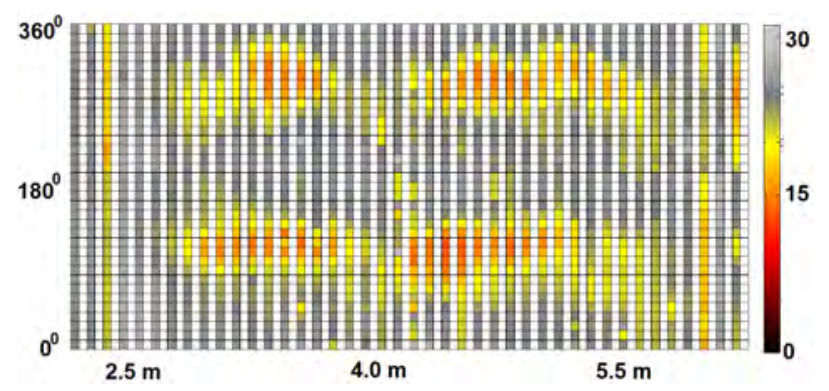

(c) Target 1060 in Fig. 17a.

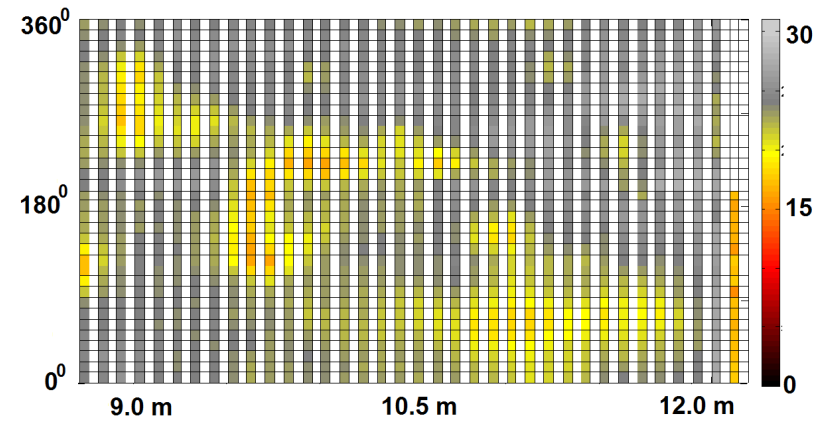

(b) Target 1670 in Fig. 15(plan) and 17b.

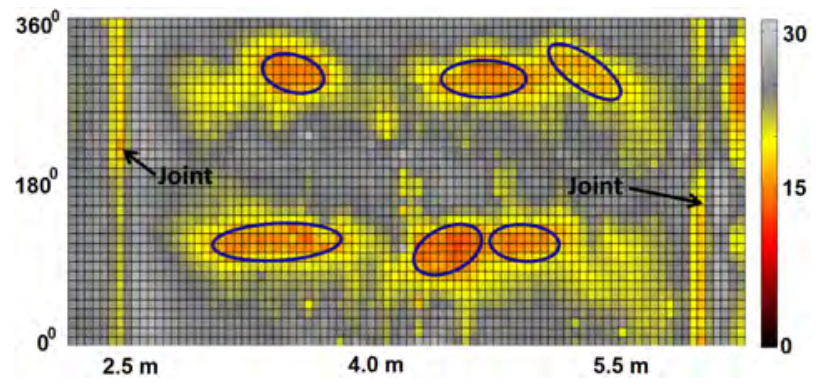

(d) GP-inferred map depicting joints and identified corrosion patches.

Figure 16: Various examples of remaining pipe wall thickness maps as measured by the robotic wall inspection during field deployment on the buried critical water main. Distances shown are with respect to the edge of closest access point.

are depicted in Fig. 17, referenced on an aerial picture showing the location where the pipeline is buried. The layout of the pipeline spool structure is also shown in yellow, where white lines are illustrative of the pipeilne diameter, and red segments identify spool joints, with the spool number inspected with the robot labelled accordingly. The date of inspection has also been added. Further details about the full program of inspections and various other tests undertaken with the NDT robot during the field deployment can be found in a comprehensive supplementary document associated to this manuscript that has been deposited in the journal webpage for the interested reader.

Prior to using the robotic tool for extensive measurements, repeatability tests were also carried out on pipe sections at the test-bed to ascertain the performance of the robotic inspection unit in-situ. Results from one of the tests are shown in Fig. 18. The error histogram in Fig. 18c suggests a close to zero-mean Gaussian $(0.112 \mathrm{~mm}$ mean, $0.869 \mathrm{~mm}$ standard deviation). Information such as minimum, maximum and average thickness of the inspected pipe section are key parameters of interest to water utilities for stress analysis and asset management in general. Table $18 \mathrm{~d}$ collects the most typical quantitative information being currently reported with the robotic device on the two inspections shown Map A (Fig. 18a) and Map B (Fig. 18b), corresponding to target 1100 in Fig. 17a.

\subsection{Pipe Inner Surface Profiling}

In addition to PEC measurements, perceptual information from video streaming and point clouds of the pipe inner surface (cement lining) can also be recorded with the RBG camera and the 3D structure sensor mounted at the front of the robot. The latter in particular allows mapping the geometry of the pipe inner surface in order to evaluate the surface unevenness, variation in the nominal pipe diameter and mapping the structure of in-pipe features (chainage, off-takes, valves). Moreover, reconstructing the inner surface profile has the advantage that it enables identifying and locating unchartered coarse anomalies present on the cement lining surface which may impede motion of ILI tools. An example of the latter was apparent during one of the inspections where the robot encountered an abnormality in the form of pipe narrowing during the experiment is shown in Fig. 19b. The mean diameter of the narrow region was 


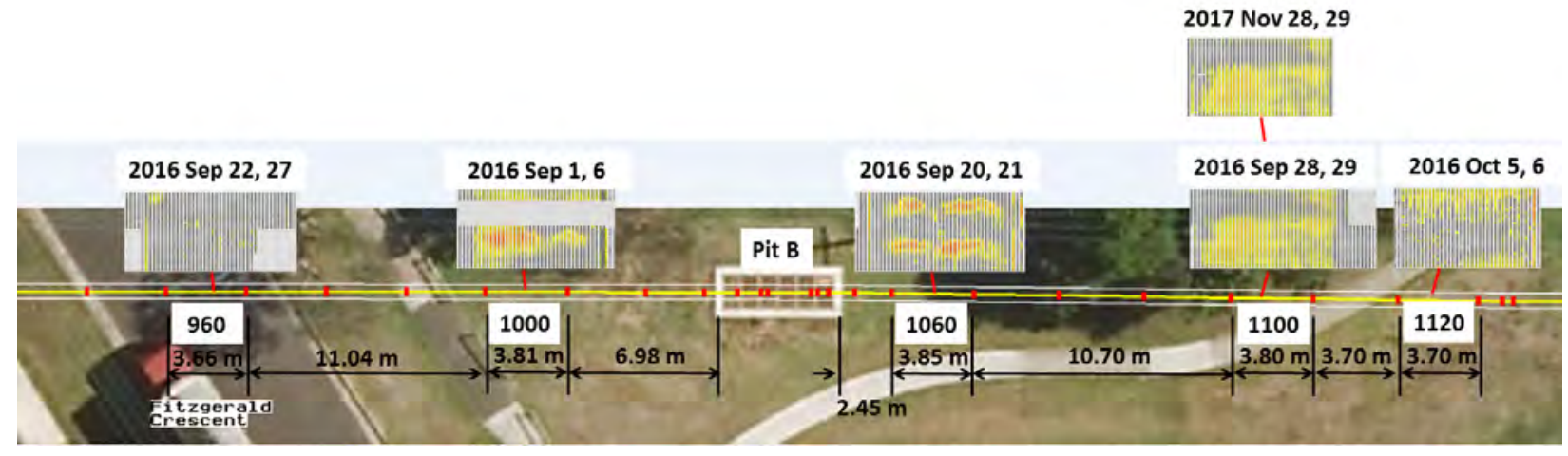

(a) Pits inspected from entry point "Pit B".

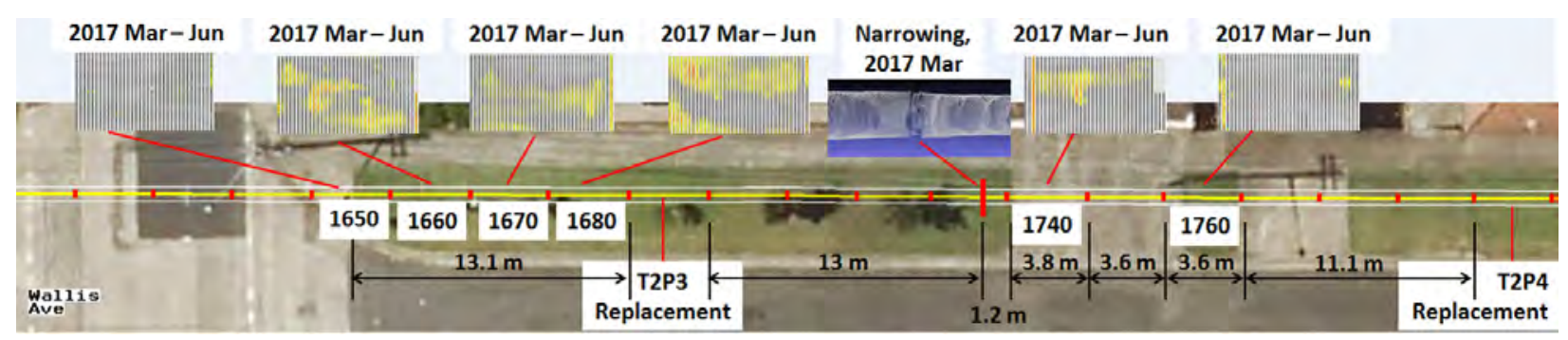

(b) Pits inspected from entry point "T2P3 Replacement".

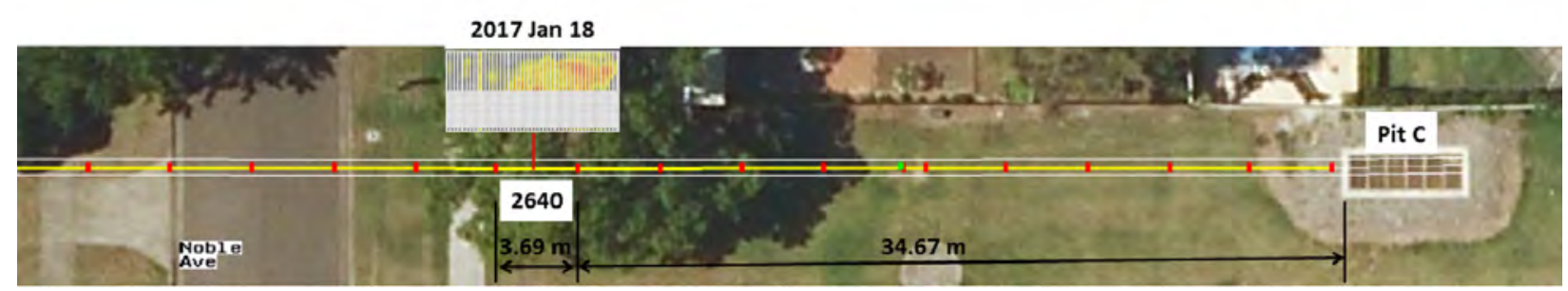

(c) Pits inspected from entry point "Pit C"..

Figure 17: Final 2.5D spool thickness maps attained from the field deployment of the NDT robotic inspection robot on a buried critical water main in Sydney during 2016-17-18. Three sections closer to the deployment entry points are shown. In the middle figure, two access points (T2P3 and T2P4) were required given the unexpected narrowing found in the pipeline (see Section 5.1).

observed to be $579 \mathrm{~mm}$ while the expected nominal diameter of the cement lined inner surface is expected to be close to $600 \mathrm{~mm}$. A posterior excavation by the utility found an unknown outer clamp as shown in Fig 19c, whose origin was investigated but not found on any records.

\section{Key Lessons Learned from the Field Deployment}

The operational aspects of interfering with a critical asset that affects large populations in their daily lives, such as the supply of drinking water, can not be understimated. The deployment of the unit on a critical pipeline was indeed a carefully orchestrated exercise. Likewise, the extraction and replacement of sections for careful examination and ground truthing was a significant undertaking in terms of logistics and resources (both personnel and monetary, including flow control, excavation, transportation, grit-blasting and pipe preservation). It was revealing the extent of consultations required with the many water utility teams that had to get involved during all phases of the project.

The actual field deployment of the robotic unit brought about some pivotal aspects to contend with, which in summary 


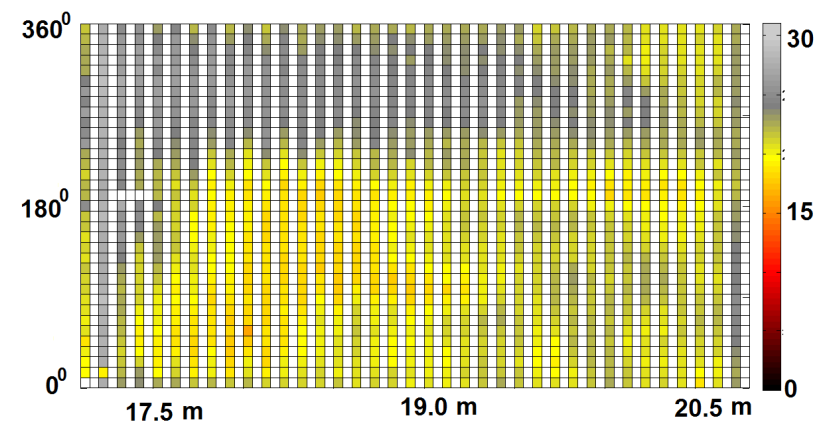

(a) Map A (Target 1100).

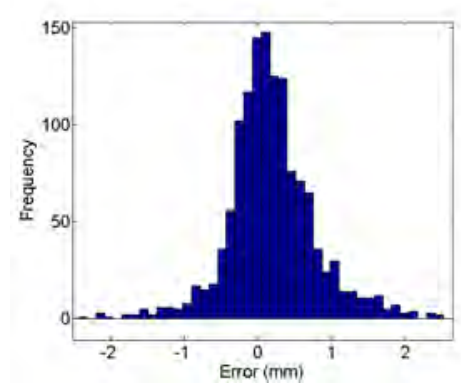

(c) Errors between maps.

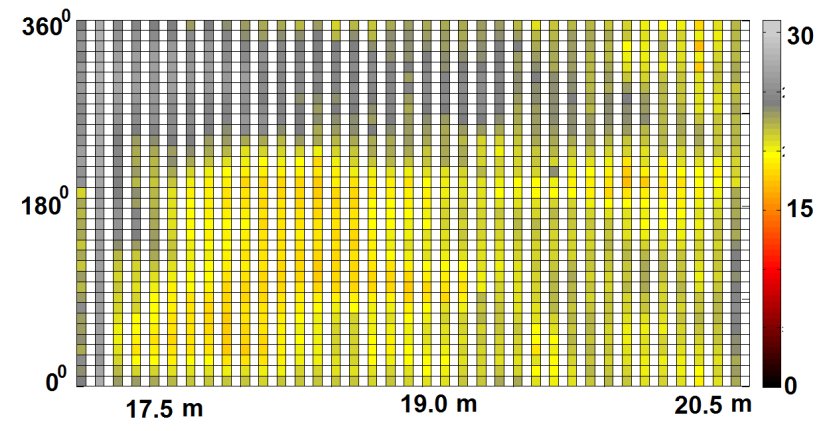

(b) Map B (Target 1100).

\begin{tabular}{lcc}
\hline & Map A & Map B \\
\hline Minimum thickness & 17.4 & 16.2 \\
Maximum thickness & 28.1 & 28.8 \\
Average thickness & 21.8 & 21.9 \\
\hline
\end{tabular}

(d) Inspection measurements (mm).

Figure 18: Robotic inspection repeatability tests on a single pipe section, target 1100 in Fig. 17a .

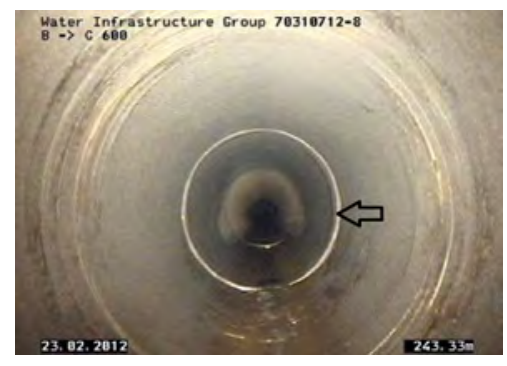

(a) Picture of anomalous narrowing (white ring at pointer).

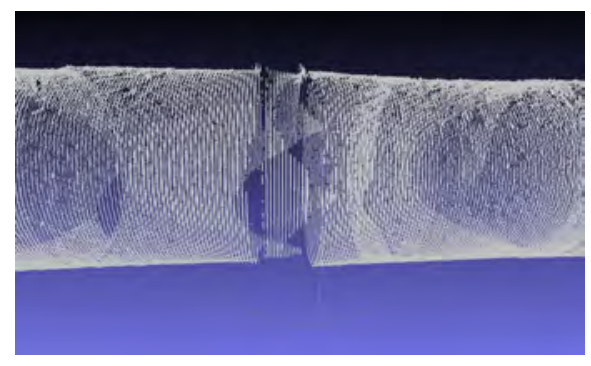

(b) 3D reconstruction of anomaly captured by RGBD robot sensor.

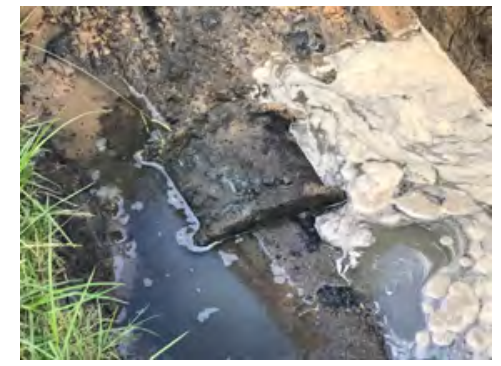

(c) Outer clamp found after excavation.

Figure 19: Unchartered pipe anomaly found during inspection; verification excavation.

could be identified as: (1) Unexpected anomalies in the pipe in the form of surface unevenness (e.g. in the rendering of the cement lining), or pipe narrowings due to unknown features (e.g. an unchartered replaced pipe); (2) Boundaries in the robustness of the hardware, given the harsh deployment conditions; (3) Impact of the limited speed on the logistics surrounding the customer support and civil delivery team efforts to be able to deploy the robot $(\sim 14$ hours to cover $50 \%$ of a $3.5 \mathrm{~m}$ long, $600 \mathrm{~mm}$ diameter pipe section); and the (4) Need to introduce a level of compliance in the sensor mountings to absorb the undocumented asymmetries and pipe manufacturing tolerances, so as to maintaining close contact with the pipe walls for NDT sensing. Specific details of various of the issues encountered in regards to the above are provided in the supplementary material document associated to this manuscript that has been deposited in the journal webpage for the interested reader.

Following the learnings drawn from entertaining these issues in the field, authors have begun investigating faster sensing architectures (multiple PEC sensors operating simultaneously) that can assess over $10 \mathrm{~m}$ of pipe length with $100 \%$ coverage (600 mm diameter) within an hour. Authors are also investigating new robot architectures which enable significant size adjustments on encountering anomalies such as reduction/enlargement in the pipe diameter 
during the inspection, or the presence (sometimes unexpectedly) of other pipe fittings and appurtenances.

Lastly, an interesting observation made during field deployment related to the actual choice of robot kinematic design, with one of the rear wheels of the robot experiencing signs of undue stress (both the left rear wheel assembly, as well as its driving motor, failed on two occasions). This was attributed to unforeseen misalignment or asymmetry in the implemented robot hardware, forcing the left rear side to experience accentuated tension. This was primarily a result of the mechanical robot design with an arm linkage to allow rotational mobility, as well as assured grip of the wheels againts the pipe walls during longitudinal motion. The lack of suspension meant that the applied forces when pressing the wheels against the wall were substantial, often inducing significant stress on the driving wheel actuators. Measures can be taken to minimize or diverge from the requirement of the whole robotic unit having to rotate by facilitating a sensing unit mechanism able to rotate while the rest of the robotic device does not, and such architectures are currently being investigated.

\section{Concluding Remarks}

An in-line robotic solution for the inspection of buried critical water mains and its evaluation during field deployments has been presented in this paper. A singular kinematic locomotion design that optimises mobility in such tubular environment has been coupled with an embedded NDT sensing solution based on PEC for measurements unsusceptible to sensor lift-off, as typically found in cement lined water pipelines. A tailored covariance function suitable for pipeline geometries has also been studied in a Gaussian Processes framework to regress missing sensor data.

The data gathered represents not only a visual understanding of the condition of the pipe for asset managers, but also constitutes a quantative input to a remaining-life calculation that defines the likelihood of the pipeline for future renewal or repair. The device addresses a utility sector need for an automatic NDT inspection vehicle that can report dense pipe wall thickness discrimination as prescribed by failure prediction analysis, and that can be deployed in an opportunistic manner - e.g. when a mains break occurs, or during valve inspection or repair programs when pipelines are discharged and access made available. Extensive results have proven the validity of the solution on laboratory tests and field pipeline inspections which demonstrate the feasibility of the device and sensing configuration to provide meaningful 2.5D geometric maps. Future work is set on further characterisation and inclussion of sensor measurement uncertainties in the analysis of the pipeline remaining-life calculations.

As highlighted in Section 6, following the learnings presented in this paper a new version of the robot is currently under development with alternative mobility and sensor arrangement to facilitate faster robotic deployment, as well as faster signal processing and acquisition. As part of that development, consideration is also being given to the unsolved problem of relating complex pipe wall geometry to a PEC signal, a somewhat unorthodox sensor in the space of probabilistic mapping. For practical purposes the NDT sensor measurements used in this work have been approximated to the average thickness under the footprint of the sensor (a $50 \mathrm{~mm} \times 50 \mathrm{~mm}$ region) with reassuring results, although in reality the sensor's measurement generalises to a domain. Due to the unsolved problem of accurately relating complex geometry to the signal, it is not trivial to assume or define a precise sensor model, and a compatible kernel to capture such behaviours for regression, a task that requires further investigation.

\section{Acknowledgement}

This work is an outcome from the Advanced Condition Assessment and Pipes Failure Prediction project (www.criticalpipes.com), an international R\&D consortium funded by Sydney Water Corporation, Water Research Foundation (USA), Melbourne Water, Water Corporation (WA), UK Water Industry Research, South Australia Water Corporation, South East Water, Hunter Water Corporation, City West Water, Yarra Valley Water, Queensland Urban Utilities, University of Technology Sydney, Monash University and University of Newcastle.

This manuscript represents an extension to (Valls Miro et al., 2017) where additional details on the PEC sensing and robot system design have been documented, alongside more detailed results of the field deployment and discussions 
fom those. Moreover, an in-depth description of a novel kernel covariance function and the GP-based algorithm employed to generate $2.5 \mathrm{D}$ thickness maps from the field data have also been added.

\section{References}

Bishop, C. M. (2006). Pattern recognition and machine learning, book Kernel Methods, pages 291-324. Information science and statistics. Springer, New York.

Davies, T. M. and Bryant, D. (2013). On circulant embedding for Gaussian random fields in R. J. Stat. Softw., 55(9):1-21.

Efron, B. and Tibshirani, R. J. (1994). An Introduction to the Bootstrap. Chapman \& Hall/CRC Monographs on Statistics \& Applied Probability. Taylor \& Francis.

Fu, F. and Bowler, J. (2006). Transient eddy-current driver pickup probe response due to a conductive plate. IEEE Transactions on magnetics, 42(8):2029-2037.

Huang, C. and Wu, X. (2015). An improved ferromagnetic material pulsed eddy current testing signal processing method based on numerical cumulative integration. NDT \& E International, 69:35-39.

Huang, C., Wu, X., Xu, Z., and Kang, Y. (2011). Ferromagnetic material pulsed eddy current testing signal modeling by equivalent multiple-coil-coupling approach. NDT \& E International, 44(2):163-168.

Huang, C., Xinjun, W., Zhiyuan, X., and Kang, Y. (2010). Pulsed eddy current signal processing method for signal denoising in ferromagnetic plate testing. NDT \& E International, 43(7):648-653.

Ji, J., Robert, D., Zhang, C., Zhang, D., and Kodikara, J. (2017). Probabilistic physical modelling of corroded cast iron pipes for lifetime prediction. Structural Safery, 64:62-75.

Kodikara, J., Valls Miro, J., and Melchers, R. (2016). Failure prediction of critical cast iron pipes. Advances in Water Research, 26(3):6-11.

Kroese, D. P. and Botev, Z. I. (2013). Spatial process generation. In Schmidt, V., editor, Lectures on Stochastic Geometry, Spatial Statistics and Random Fields, Volume II: Analysis, Modeling and Simulation of Complex Structures. Springer-Verlag.

Liu, Z. and Kleiner, Y. (2013). State of the art review of inspection technologies for condition assessment of water pipes. Measurement, 46(1):1-15.

Lord, G. J., Powell, C. E., and Shardlow, T. (2014). An Introduction to Computational Stochastic PDEs, chapter Random Fields, pages 257-310. Cambridge University Press.

O’Callaghan, S. and Ramos, F. (2012). Gaussian process occupancy maps. I. J. Robotic Res., 31(1):42-62.

Rasmussen, C. E. and Williams, C. K. I. (2006). Gaussian Process for Machine Learning, chapter Regression, pages 7-32. MA:MIT press, Cambridge.

Schlather, M., Malinowski, A., Menck, P. J., Oesting, M., and Strokorb, K. (2015). Analysis, simulation and prediction of multivariate random fields with package randomfields. J. Stat. Softw., 63(8):1-25.

Shi, L. and Valls Miro, J. (2017). Towards optimised and reconstructable sampling inspection of pipe integrity for improved efficiency of non-destructive testing. Water Science and Technology: Water Supply.

Skinner, B., Vidal-Calleja, T., Valls Miro, J., De Bruijn, F., and Falque, R. (2014). 3D point cloud upsampling for accurate reconstruction of dense 2.5D thickness maps. In Australasian Conference on Robotics and Automation, page 7 .

Smith, M., Posner, I., and Newman, P. (2010). Efficient non-parametric surface representations using active sampling for push broom laser data. In Robot. Sci. Syst. (RSS). 
Sun, L., Vidal-Calleja, T., and Valls Miro, J. (2015). Bayesian fusion using conditionally independent submaps for high resolution 2.5 D mapping. In IEEE Int. Conf. Robot. Autom. (ICRA), pages 3394-3400.

Taheri, H., Qiao, B., and Ghaeminezhad, N. (2015). Kinematic model of a four mecanum wheeled mobile robot. International Journal of Computer Applications, 113:6-9.

Tartakovsky, D. M. and Xiu, D. (2006). Stochastic analysis of transport in tubes with rough walls. Journal of Computational Physics, 217(1):248-259.

Tesch, M., Schneider, J., and Choset, H. (2011). Using response surfaces and expected improvement to optimize snake robot gait parameters. In IEEE/RSJ International Conference on Intelligent Robots and Systems (IROS), pages 1069-1074. IEEE.

Ulapane, N., Alempijevic, A., Vidal-Calleja, T., Valls Miro, J., Rudd, J., and Roubal, M. (2014). Gaussian process for interpreting pulsed eddy current signals for ferromagnetic pipe profiling. In Conference on Industrial Electronics and Applications, pages 1762-1767.

Ulapane, N., Nguyen, L., Valls Miro, J., and Dissanayake, G. (2017). Designing a pulsed eddy current sensing setup for cast iron thickness assessment. In Conference on Industrial Electronics and Applications, pages 892-897. IEEE.

Valls Miro, J., Hunt, D., Ulapane, N., and Behrens, M. (2017). Field and Service Robotics, Results of the 11th International Conference, chapter Towards Automatic Robotic NDT Dense Mapping for Pipeline Integrity Inspection, pages 319-333. Springer Proceedings in Advanced Robotics.

Valls Miro, J., Rajalingam, J., Vidal-Calleja, T., de Bruijn, F., Wood, R., Vitanage, D., Ulapane, N., Wijerathna, B., and $\mathrm{Su}, \mathrm{D}$. (2014). A live test-bed for the advancement of condition assessment and failure prediction research on critical pipes. Water Asset Management Intl., 10(2):3-8.

Vasudevan, S., Ramos, F., Nettleton, E., Durrant-Whyte, H., and Blair, A. (2009). Gaussian process modeling of large scale terrain. In IEEE Int. Conf. Robot. Autom. (ICRA), pages 1047-1053.

Vidal-Calleja, T., Su, D., De Bruijn, F., and Valls Miro, J. (2014). Learning spatial correlations for bayesian fusion in pipe thickness mapping. In Robotics and Automation (ICRA), 2014 IEEE International Conference on, pages 683-690. IEEE.

Xie, L., Scheifele, C., Xu, W., and Stol, K. A. (2015). Heavy-duty omni-directional mecanum-wheeled robot for autonomous navigation: System development and simulation realization. In International Conference on Mechatronics, pages 256-261. IEEE.

Xu, Z., Wu, X., Li, J., and Kang, Y. (2012). Assessment of wall thinning in insulated ferromagnetic pipes using the time-to-peak of differential pulsed eddy-current testing signals. NDT \& E International, 51:24-29. 


\title{
Robotic Pipeline Wall Thickness Evaluation for Dense NDT Inspection
}

\author{
Jaime Valls Miro, Nalika Ulapane, Lei Shi, Dave Hunt and Michael Behrens \\ Centre for Autonomous Systems, University of Technology Sydney, \\ \{jaime.vallsmiro, nalika.ulapane, lei.shi, dave.hunt, michael.behrens\}@uts.edu.au
}

\begin{abstract}
This paper addresses automated mapping of the remaining wall thickness of metallic pipelines in the field by means of an inspection robot equipped with Non-Destructive Testing (NDT) sensing. Set in the context of condition assessment of critical infrastructure, the integrity of arbitrary sections in the conduit is derived with a bespoke robot kinematic configuration that allows dense pipe wall thickness discrimination in circumferential and longitudinal direction via NDT sensing with guaranteed sensing lift-off (offset of the sensor from pipe wall) to the pipe wall, an essential barrier to overcome in cement-lined water pipelines. A tailored covariance function for pipeline cylindrical structures within the context of a Gaussian Processes has also been developed to regress missing sensor data incurred by a sampling strategy followed in the field to speed up the inspection times given the slow response of the PEC electromagnetic sensor proposed. The data gathered represents not only a visual understanding of the condition of the pipe for asset managers, but also constitutes a quantative input to a remaining-life calculation that defines the likelihood of the pipeline for future renewal or repair. Results are presented from deployment of the robotic device on a series of pipeline inspections which demonstrate the feasibility of the device and sensing configuration to provide meaningful $2.5 \mathrm{D}$ geometric maps.
\end{abstract}

\section{Motivation - A Taxonomy of NDT Inspection Techniques}

Non-Destructive Testing (NDT) or Evaluation (NDE) is extensively employed by the energy and water industry to assess the integrity of their network assets, particularly their larger and most critical conduits (generally refered to 


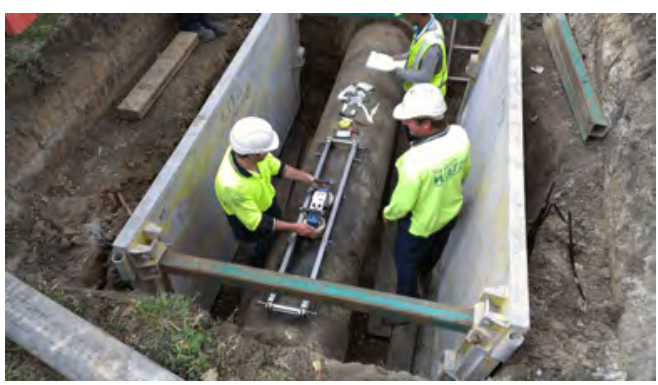

(a) Externa locall inspection tool.

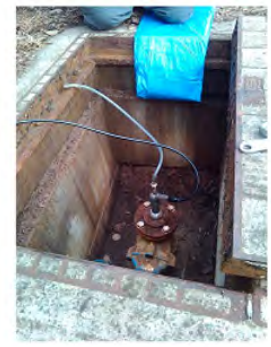

Water Pressure Sensor 2

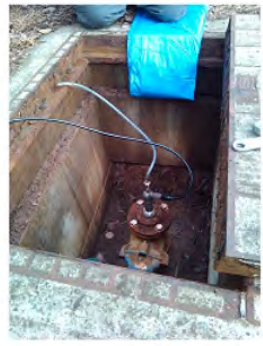

Water Pressure

Sensor 1

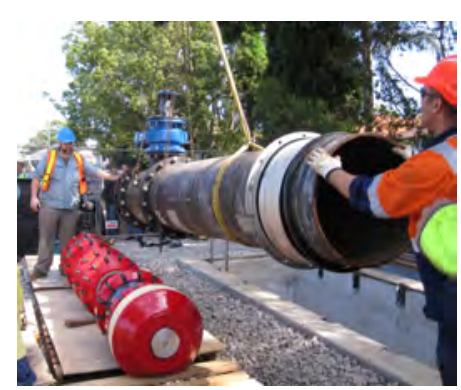

(b) ILI tool.

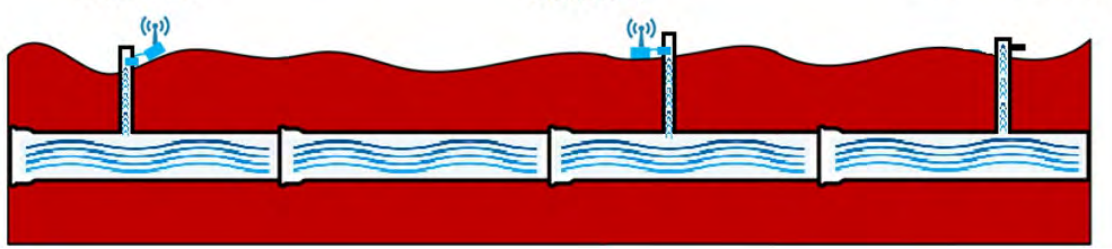

(c) An external averaging tool.

Figure 1: Example of various configurations of NDT tools.

as those larger than $350 \mathrm{~mm}$ in diameter), in their decision-making process leading their renewal/repair/rehabilitation programs. The key advantage of NDT/NDE is that the structure of the asset is not compromised in estimating its condition.

The sensing modality to use is strongly influenced by the material of the asset. Grey Cast Iron (CI) pipelines remain the bulk of the buried critical water infrastructure in the developed world as that was the material of choice for mass production with the advent of the Industrial Revolution in the middle of the 18th century (alongside its less brittle relative of Ductile Iron since the nineteen fifties), until carbon steel, asbestos cement or plastic pipelines (PVC) amongst other materials made them redundant over the years. The non-homogeneity of the CI produce means that sensing techniques widely employed in the (mild) Carbon Steel networks in the energy pipeline sector, such as ultrasonics or electromagnetic acoustic transducers (EMAT), are inadequate for CI, and the underlying techniques of most commercial propositions for CI are instead based on either magnetics (e.g. Magnetic Flux Leakage (MFL), Pulsed Eddy Currents (PEC) and Remote Field Eddy Currents (RFEC)), or the study of the propagation of pressure waves in the 
pipeline and/or fluid.

NDT techniques produce results that tend to be a trade-off between deployment costs and information gain. Local inspection techniques (i.e. 1 to 3 meters) can provide dense measurements but are time-consuming and generally costly per unit-length as significant preparatory civil works are required (excavations, network re-routing for guaranteed supply, traffic control, etc). Moreover inspections can only be undertaken at locations which are accesible from the surface. An example of these tools can be seen in Fig. 1a.

On the other hand, the taxonomy of long-coverage tools can be broadly split into techniques that provide average pipe wall measurements over longer distances (generally from a few to 100s of meters, even kilometers), and in-line intrusive (ILI) devices ("smart pigs") deployed inside the pipeline to inspect in higher detail over longer distances (generally 100's of meters to kilometers to make it more cost-effective), while propelled by the operating pressure of the fluid.

The former are generally deployed by accessing the external pipe wall or water column at a few access points spread over the length of the pipeline, either through small key-hole excavations or through external access points such as valves or hydrants. As such they tend to have low or no impact in the continuing operation of the pipeline and are more affordable alternatives for condition assessment. An example can be seen in Fig. 1c. However given the averaging nature of their results, these tools are aimed at providing an initial screening of the condition of an asset, and lack the ability to provide the type of detailed geometry information needed to ascertain likelihood of pipe failure.

Flow-driven ILI tools, on the other hand, are inserted into the charged water column either through standard large appurtenances present in critical mains, or more often than not via dedicated launch and retrieval mechanisms, as depicted in Fig. 1b. While these tools are able to provide direct measurements related to the pipe wall condition over long distances, they do so at the expense of higher disruption to the utilities and combined costs from the substantial civil engineering support from the utility prior, during and post inspection. Moreover, the effectiveness of these techniques has not been fully established within the industry given the consequential validation investment required to do so in a statistical meaningful way.

ILI tools present additional shortcomings in the pursuit of attaining an accurate depictions of the condition of a pipe wall:

- they are at the mercy of the pressure of the fluid driving them (both in the tethered and free-flowing case).

- should the tools be operated in de-watered conditions, they necessitate complicated winch mechanisms be- 
tween entry and exit points.

- operating parameters need to be closely controlled (e.g. tool velocity), meaning that discriminating flow controls need to be in place, not necessarily an easy feat to achieve in a complex interconnected network.

- they lack the ability to do fine control and adjustments for mapping (e.g. ensuring tight tolerances in sensor lift-off, repeatability, rectify missed measurements).

Driven by the needs of the water industry the work hereby presented describes the development and field testing of a novel internal NDT inspection robotic vehicle able to:

1. undertake localised, controlled inspections.

2. generate dense NDT mapping suitable for condition assessment and failure prediction.

3. tightly control inherent lift-off during sensing (as induced by the presence of non-magnetic cement lining and pipeline wall irregularities)

4. access arbitrary (within tether range) pipeline spools from a single point of entry, hence reducing costs to utilities and allowing inspection of inaccessible sections from the surface (e.g. under a rail pass) and minimising disruption to customer (e.g. a pipeline under a driveway).

While the proposed solution requires pipes to be de-watered for deployment, this serves a clear mandate from the utility sector that necessitates a robotic NDT inspection vehicle that can be deployed in an opportunistic manner to ascertain the condition of a particular pipeline, specifically when a mains break occurs, or on the back of a valve inspection or repair program when pipelines are inevitably taken off-line. Moreover, while time is always at essence in any maintenance and inspection routines, this is particularly the case for critical assets that need to be put back on-line as soon as feasibly possible. To that end, an efficient robotic inspection solution with the ability to produce detailed dense maps fitting for pipe failure analysis from limited sets of inspection data is highly desirable as that would minimise both collection time and information losses against the original wall thickness maps. The use of Gaussian Processes is thus proposed in this work to model the spatial dependencies present in the interspersed thickness data collected, and to recover form these the required detailed pipe wall geometries.

The remainder of this paper describes such an NDT robot for the inspection of buried network infrastructure and the novelties behind its inception. The manuscript is organized in two main parts. The first part covers the hardware design which includes Section 2, where the sensing capabilities used to measure the pipeline wall thickness are first 


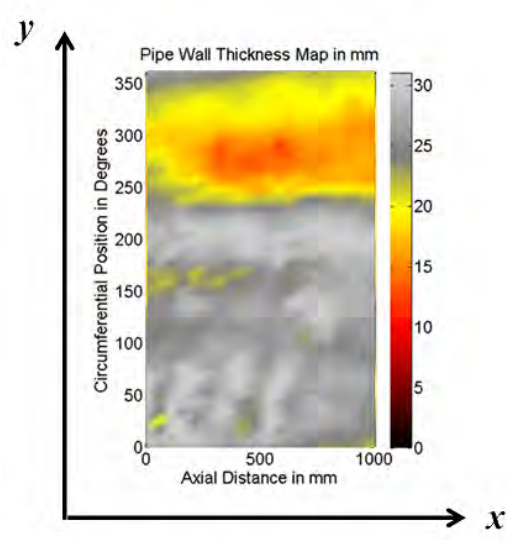

(a) Axis of $2.5 \mathrm{D}$ thickness map.

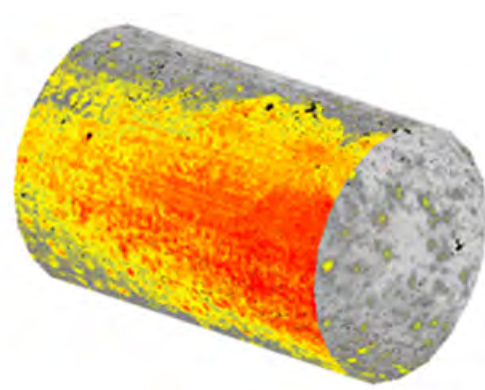

(b) Rolled pipe thickness map.

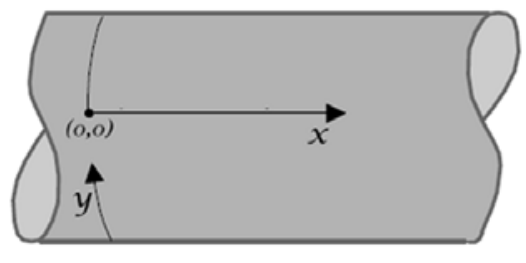

(c) Axial $x$ and circumferential $y$ coordinates aligned on a pipe.

Figure 2: Axial and circumferential coordinates of a 2.5D pipe thickness map.

established, and Section 3 where the locomotion choices and controls for the proposed robotic platform given the specifics of a pipeline environment are then described. The second part describes the Gaussian Process model used to generate maps of wall thickness based on the measurements taken by the robotic platform, described in Section 4, linked to the actual detailed inspection results from deployment in the network of a water utility given in Section 5. Learnings from the development of the robot and the field deployment have been discussed in Section 6 in the hope that those insights may aid interested readers pursuing related ouctomes, whilst concluding remarks and further work are then collected in Section 7.

\section{NDT Pipeline Wall Inspection}

Recent research in the space of stress analysis and failure prediction of critical CI water mains has revealed that over and above pit depths, as traditionally provided during condition assessment of a critical asset, there is a need to ascertain the presence and geometries of large corrosion patches in the pipe walls (Ji et al., 2017; Kodikara et al., 2016), such as those depicted in Fig 16d. There exist a wide range of NDT technologies developed for the purpose of material characterisation for CI (Liu and Kleiner, 2013), yet the provision to build dense 2.5D maps of remaining wall geometries for lined water mains has driven the need to design an internal inspection tool around Pulsed Eddy Current (PEC) sensing technology, as a proven technique typically used in the NDT sector for ferromagnetic material thickness estimation (Huang et al., 2010; Xu et al., 2012; Huang et al., 2011), resilient to sensor lift-off. It is noteworthy to emphasise that while there are a myriad of commercial NDT tools available, they are mostly aimed at visual inspection of an asset, or target carbon steel with the ultimate aim to poinpoint single pitting deficiencies reliably. To date, the 


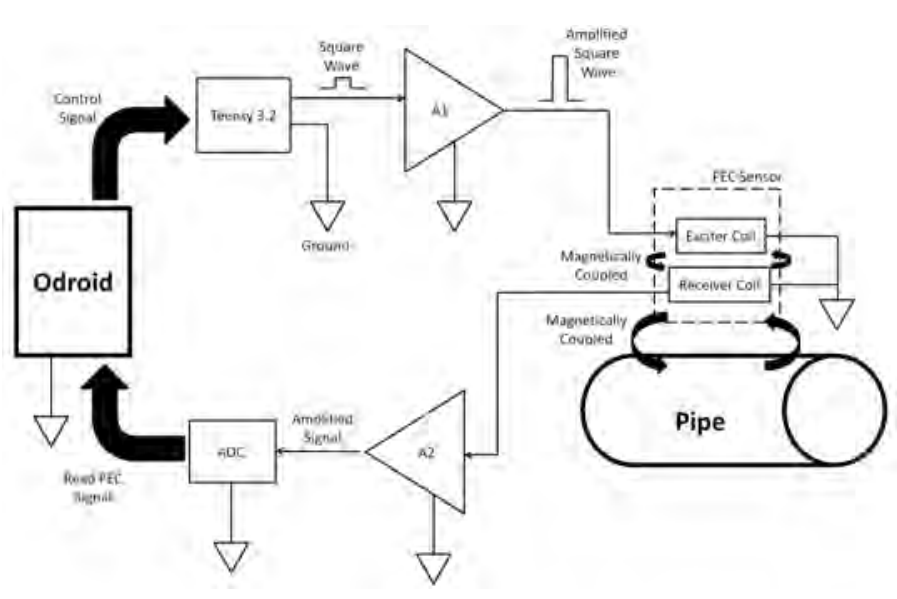

(a) PEC sensing operating diagram.

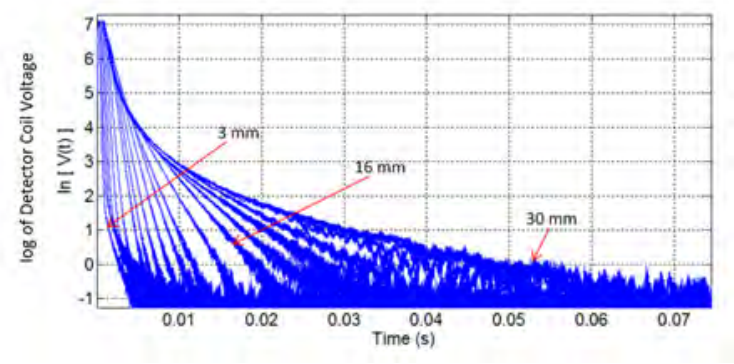

(b) Typical PEC signals on CI thicknesses.

Figure 3: PEC sensing setup embedded in the inspection robot (left), and typical PEC signals.

authors are not aware of any NDT tools able to densely map arbitrary spools of thick CI material, as it is the intention in this work.

Fig. 2 enables interpreting a typical 2.5D maps of remaining wall thickness as depicted throughout this article, and the conventions shown hold for all thickness maps presented herein. The axial location indicates the distance along the pipe's longitudinal axis, while the circumferential location represents rotational degrees around the pipeline. $0^{\circ}$ and $360^{\circ}$ coincide on the top (crown) of the pipe, denoting the direction pointing vertically upwards perpendicular to the pipe's cylinder axis. As shown in Fig. 2c, the direction of Y-axis increment is counter-clockwise when looking towards the positing $x$ direction, or the direction of robot's travel. It should be noted that despite the visual representation of the circumferential dimensions in degress throughout the paper to aid the reader's intuitive understanding of cilyndrical measurements around a pipeline, in mapping terms they are treated as 2D length measurements in $\mathrm{mm}$ in the Euclidean space, for which a single thickness measurement is obtained via the PEC sensor (hence 2.5D maps). The colour bar to the right of the thickness maps is a legend representing thickness in $\mathrm{mm}$, between black ( $0 \mathrm{~mm}$, or a through-hole) and light grey $(30 \mathrm{~mm})$. In the field inspection results presented in Section 5, longitudinal locations are in reference to the origin set at the robot's entry point to the pipe.

\subsection{Developed PEC Sensing System}

A typical PEC sensing system developed for ferromagnetic materials consist of an exciter coil, a detector coil, a voltage pulse generator for excitation and an amplifier for the detected signal. A block diagram of the PEC sensing set up developed for this work is shown in Fig. 3a. Given the size of the pipes of interest the footprint of the sensor used was $50 \mathrm{~mm}$, indicating that it measures the average thickness of a $50 \mathrm{~mm} \times 50 \mathrm{~mm}$ area under the sensor. An 


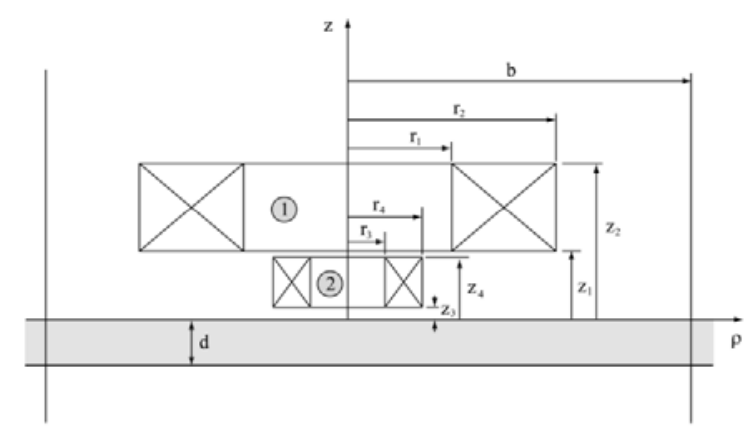

(a) Cross section of a typical coil based circular PEC sensor (adapted from (Fu and Bowler, 2006)).

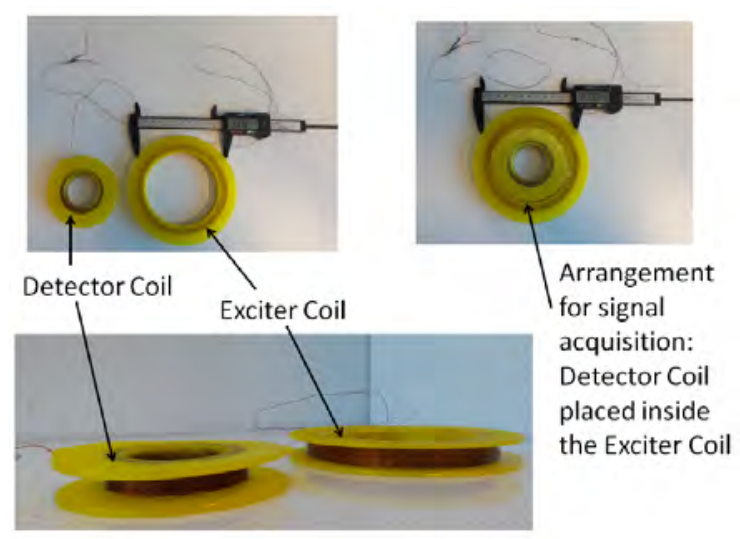

(b) PEC sensor for cast iron pipe assessment (Ulapane et al., 2017).

Figure 4: Typical coil based circular PEC sensor architecture.

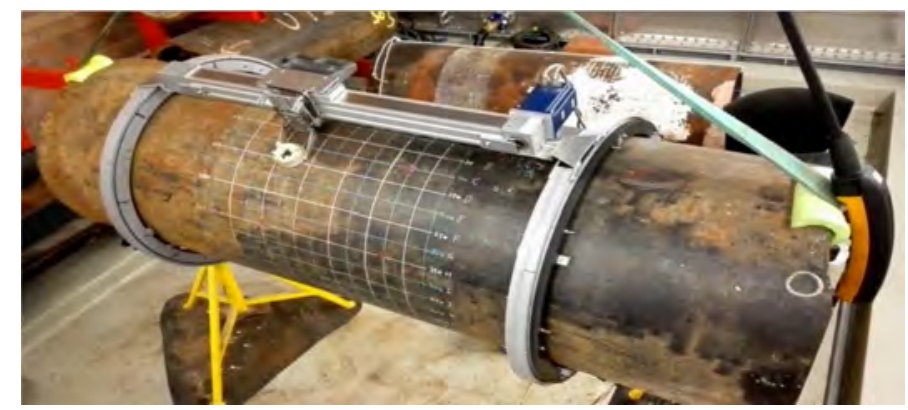

(a) Exhumed pipe on which internal and external measurements were performed.

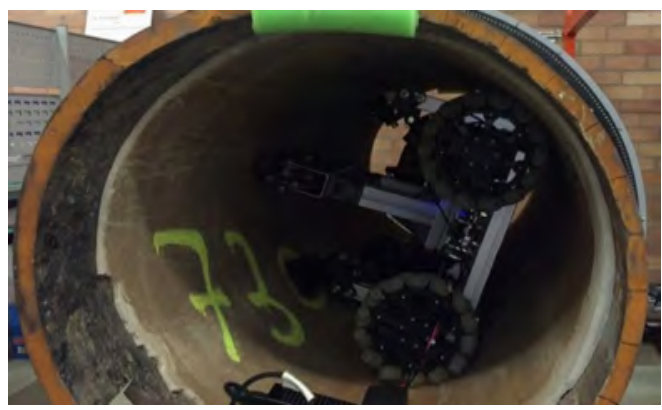

(b) Pipe assessment robot performing internal measurements.

Figure 5: Laboratory setup with exhumed pipe for internal and external PEC validation.

example of a typical PEC sensorcoil arrangement is illustrated in Fig. 4. Signals captured from the system on different CI thicknesses are shown in Fig. 3b and as reported in the literature features can be extracted from such signals which can be directly linked to material thickness (Huang and Wu, 2015; Ulapane et al., 2014; Ulapane et al., 2017).

\subsection{Validation of PEC Robot Sensor Setup}

The validity of the sensor arrangement was first assessed by comparing results on the exhumed CI pipe in Fig. 5 with intact cement lining. The objective was to evaluate how well the measurements agree if a section of the pipe is scanned externally and internally via cement lining. External measurements were performed on known locations with the aid of the grid pattern marked in Fig. 5a. The same locations were scanned internally as shown in Fig. 5b with the aid of the robot localized with reference to the pipe's edge. Measurements were recorded by placing the sensor centred on the grid squares at $50 \mathrm{~mm}$ distance increments along rings in the circumferential direction, whilst distance between 


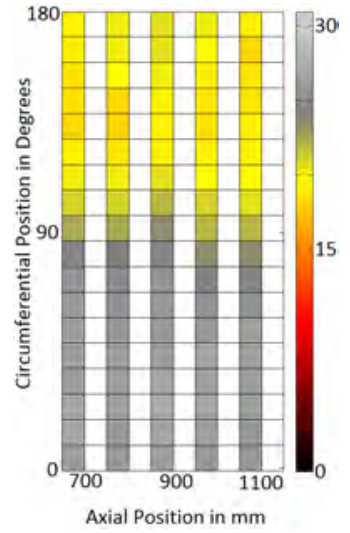

(a) External.

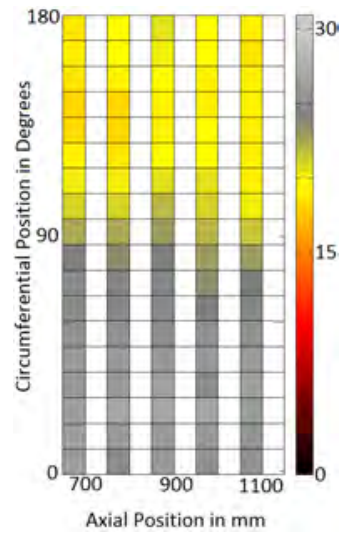

(b) Internal.

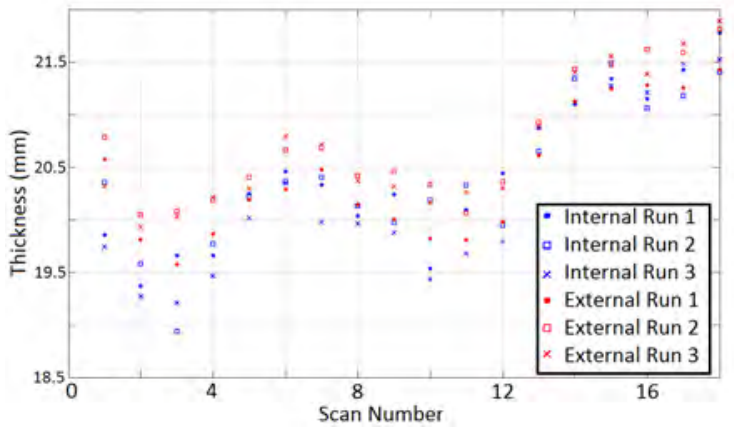

(c) Repeatability of straight line test.

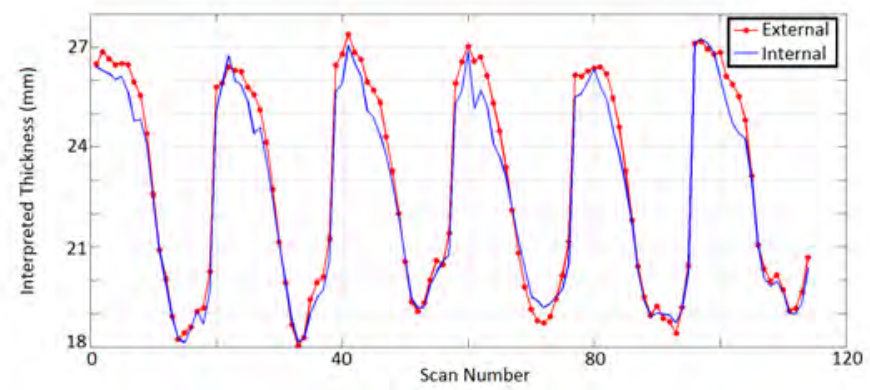

(d) One-on-one comparison 6a vs. 6 b.

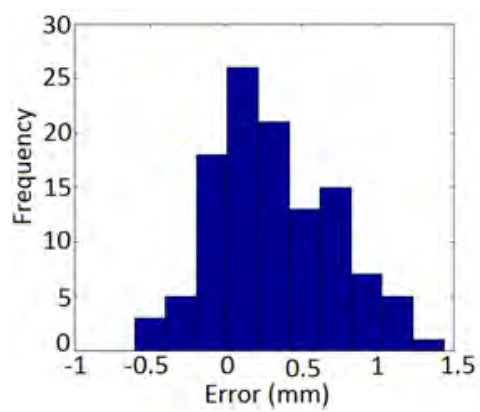

(e) Error histogram 6a vs. 6b.

Figure 6: Thickness maps obtained from internal (in blue) and external (in red) PEC sensor measurements to contrast the validity of an internal sensor deployment mounted on the robotic device when operating through the internal layer of cement lining. Two examples are provided, a rectangular section $\left(500 \mathrm{~mm}\right.$ longitudinally, and $180^{\circ}$ circumferentially) - collected in 6a, 6b, 6d and 6e, and an internal versus external repeatability straight line test shown in $6 \mathrm{c}$.

consecutive rings was set to $100 \mathrm{~mm}$ to speed-up the inspection process, since thus generated thickness maps can be then upsampled with minimal information loss as will be shown in Fig. 16d. The rationale and methodology for this will be further elaborated on in the following two Sections. Strong agreement between both measurements was notable as depicted in Fig. 6; the error histogram in Fig. 6e, calculated by subtracting internal thickness estimates from external ones hints at a small positive bias in the error, with a mean and standard deviation of $0.323 \mathrm{~mm}$ and $0.417 \mathrm{~mm}$ respectively. This is an expected result since marginally better sensitivity can be expected when scanning externally (particularly for higher thickness), as the sensor touching the pipe wall can achieving stronger penetration than from the inside given the lift-off effect induced form the cement lining layer. The errors are indicative of acceptable agreement between internal and external measurements confirming the sensor's suitability for internal assessment of pipes via cement lining. In another sensor verification experiment, Fig. 6c shows results of a repeatability test carried by measuring a straight line along the pipe six times (three times internally and three times externally, with each line having 18 measurements); the average variation on a location was less than $1 \mathrm{~mm}$, indicating appreciable measurement repeatability. 


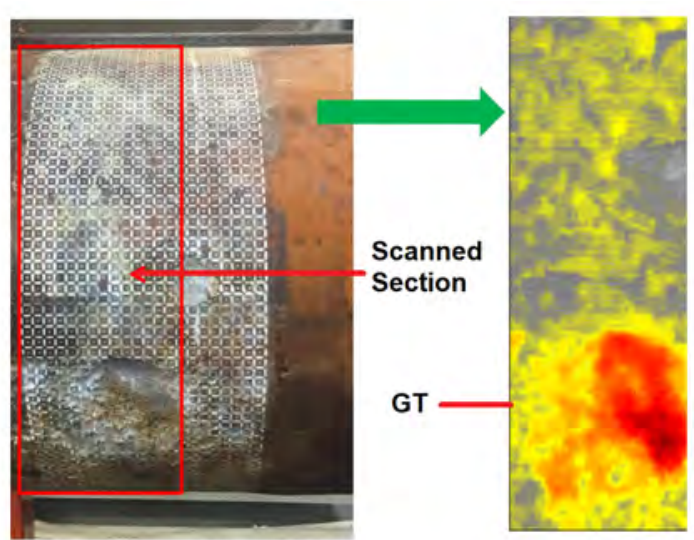

(a) Pipe section and high resolution GT map.

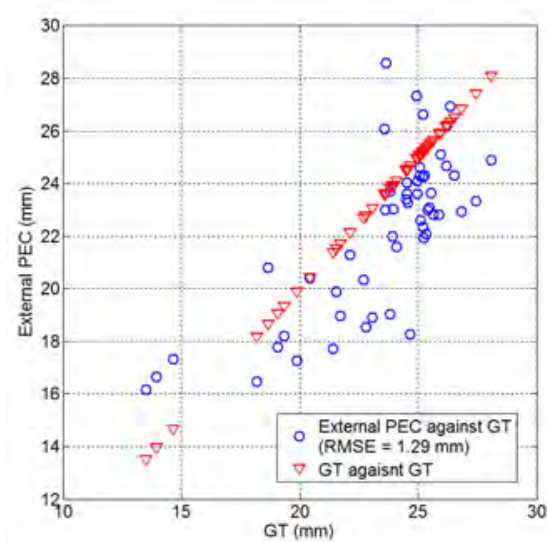

(b) Estimates against laser GT.

Figure 7: External PEC thickness estimates against laser groud truth (GT).

Further to the comparison of internal versus external deployment, the PEC sensor arrangement was also validated on a pipe whose actual wall thickness ground truth (GT) had been previously obtained, with the results collected in Fig. 7. Attaining the GT is a destructive process, whereby the pipes are first exhumed, then both internal and external pipe surfaces are grit-blasted to remove rust and graphitization, the by-products of the corrosion process inflicted on a buried pipeline, leaving only the bare metal - the target of the PEC sensor measurement. Both surfaces are then reconstructed with a high-resolution 3D laser scanner and ray-tracing performed on the collocated upsampled internal and external pipe surface point clouds to derive the GT thickness maps at a resolution of $0.6 \mathrm{~mm}$ (Skinner et al., 2014). This high resolution GT can then be downsampled to the sensor's $50 \mathrm{~mm}$ footprint by means of averaging so as to match the PEC sensor measurements in order to provide meaningful comparisons. A Root Mean Square Error (RMSE) of $1.29 \mathrm{~mm}$ was observed between external PEC measurements and GT, indicating reasonable agreement even when challenged by significant defects as evident from the testing pipe depicted in Fig. 7a, selected to better capture variability in the remaining wall thickness.

\section{NDT Robot Kinematics, Locomotion and Control}

The robotic NDT mapping unit was designed to allow accurate positioning of sensors internally on the pipe surface, in a robust and repeatable manner. To achieve this, a mechanism designed to self-align inside the pipe while providing circumferential and longitudinal control with a single actuation to place sensors against the pipe inner wall was developed. The CAD model and a deployment in the field after construction is depicted in Fig. 8. 


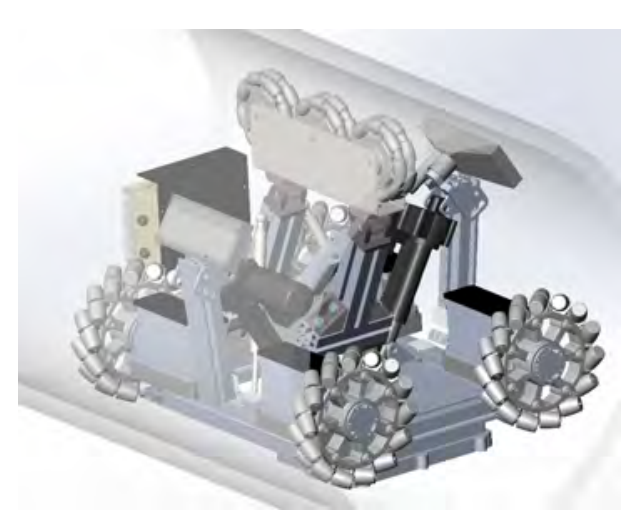

(a) Solid model design.

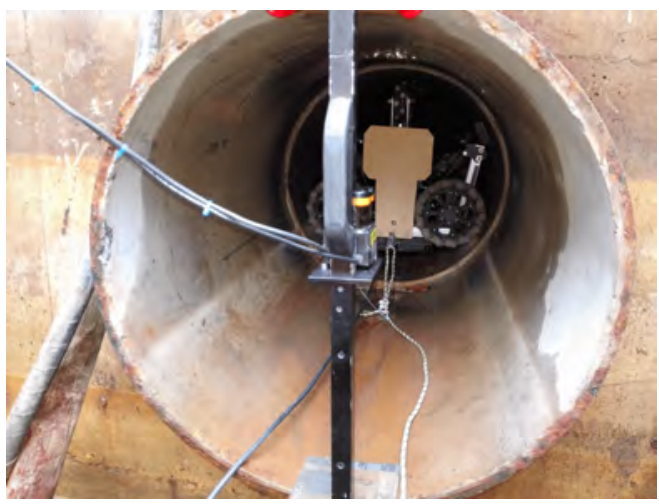

(b) Robot inspecting a pipeline.

Figure 8: NDT inspection robot design, and during field deployment in a pipeline.

\subsection{Mechanical Design}

Mecanum wheels were selected for the robot locomotion. In planar applications they enable holonomic robot motion as they allow control in all three degrees-of-freedom (DoF) available to the robot (Xie et al., 2015). For this application it is only necessary to control two degrees-of-freedom, longitudinal and circumferential motion. By applying a nonstandard wheel configuration it is possible to exploit the unique geometry of the operating environment to passively align with the central pipe axis, automatically tracking the pipe should minor changes in direction occur. Fig. 9a and Fig. $9 \mathrm{~b}$ demonstrate the layout designed to achieve these requirements. In this configuration, the axis of rotation of the pipe contacting rollers all pass through a single point allowing the robot to rotate freely about this point in response to an external force. When resting on a cylindrical surface, such as a pipe wall, an external restoring force is generated in response to angular disturbances which acts to return the robot to the aligned position.

Control in the longitudinal direction and rotation about the circumferential direction are achieved by controlling wheel velocities using the kinematic relations derived in Eq. 1, which follow standard forwards kinematic equations in simplified form (Taheri et al., 2015), where $v_{\mathrm{x}}(t)$ reflects the longitudinal velocity $(\mathrm{m} / \mathrm{s}), v_{\mathrm{y}}(t)$ is the circumferential velocity $(\mathrm{m} / \mathrm{s}), \omega_{i}(i=1 \ldots 4)$ is the wheel rotation speed $(\mathrm{rad} / \mathrm{s}), \omega_{\mathrm{z}}(t)$ denotes angular velocity on the $\mathrm{x} / \mathrm{y}$ plane, $r$ is the wheel radius $(\mathrm{m})$ and $l_{\mathrm{x}}, l_{\mathrm{y}}$ indicate the wheel separation and body length respectively.

$$
\begin{array}{r}
v_{\mathrm{x}}(t)=\left(\omega_{1}+\omega_{2}+\omega_{3}+\omega_{4}\right) \times \frac{r}{4} \\
v_{\mathrm{y}}(t)=\left(-\omega_{1}+\omega_{2}+\omega_{3}-\omega_{4}\right) \times \frac{r}{4} \\
\omega_{\mathrm{z}}(t)=\left(-\omega_{1}+\omega_{2}-\omega_{3}+\omega_{4}\right) \times \frac{r}{4 \times\left(l_{\mathrm{x}}+l_{\mathrm{y}}\right)}
\end{array}
$$



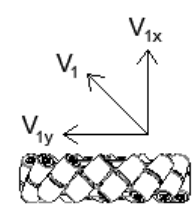

$\omega_{1}$

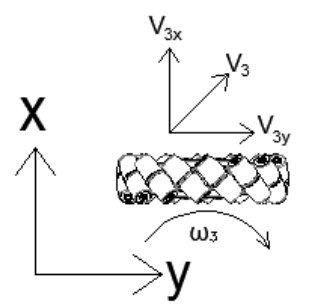

(a) Top view
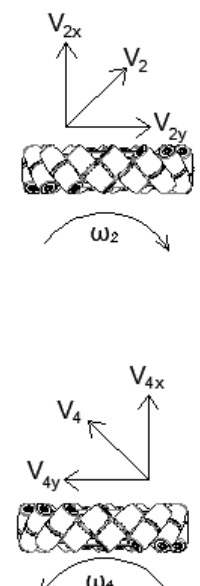

$\omega_{4}$

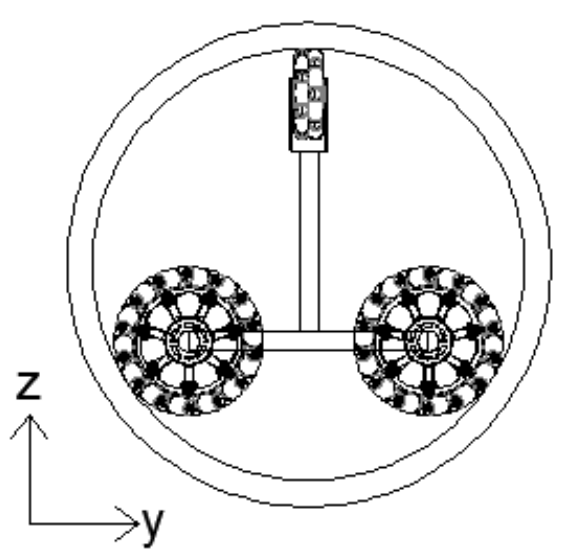

(b) Front view.

Figure 9: Adopted self-alignment mecanum wheel layout. Longitudinal wheel spacing along the $\mathrm{x}$-axis prevents angular motion about the $\mathrm{z}$-axis, therefore maintaining a heading along the longitudinal pipeline direction. The pipe wall stops the robot translating horizontally along the y-axis - instead resulting in the full circumferential rotation desired for the pipe inspection task given the additional support of the deployed vertical stability arm as shown in $9 \mathrm{~b}$, which also allows for consistent surface contact of all the mecanum rollers (at the expense of increased friction with the pipe walls).

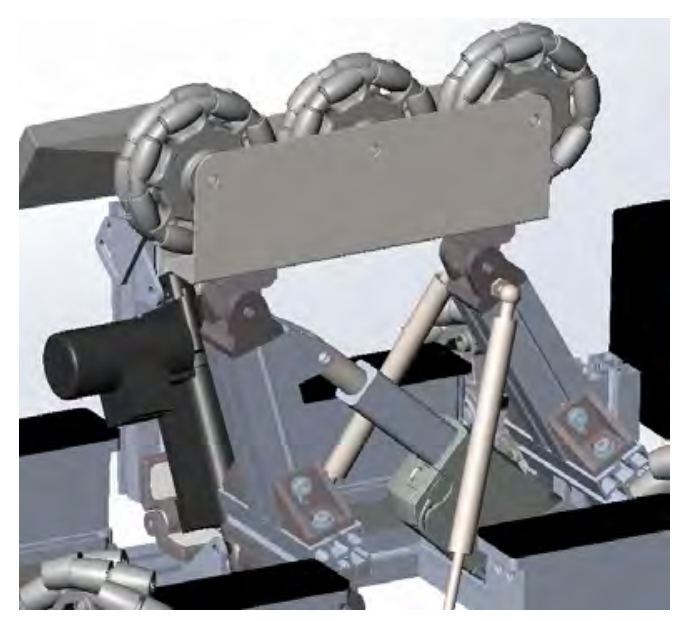

(a) Arm design.

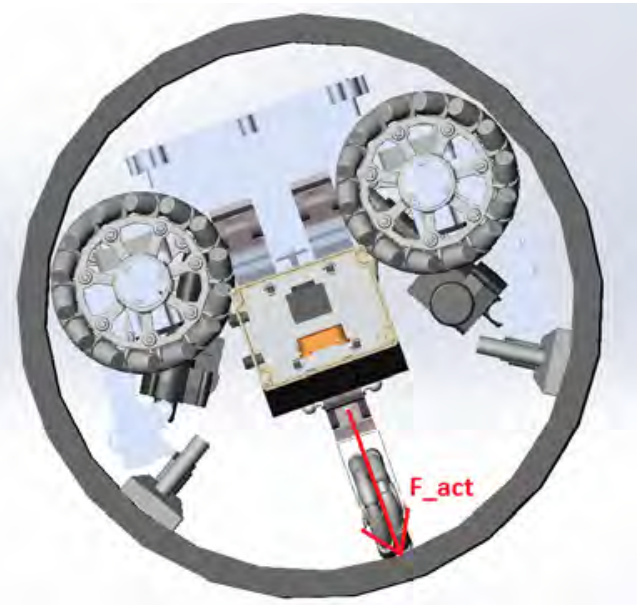

(b) Rotated stabilising arm.

Figure 10: Stabilising arm.

It is evident from Eq. (1) that maintaining zero angular velocity (and therefore a constant heading) is a wheel speed control task. As demonstrated in Fig. 9b, wheel spacing prevents angular motion about the $\mathrm{z}$ axis, therefore maintaining a heading along the longitudinal direction. The pipe wall stops the robot translating horizontally - instead resulting in the circumferential rotation that is desired for this task. It is however essential that the angular velocities of diagonally opposite wheels are matched to prevent excessive motor loads precisley given the robot is constrained in the $\mathrm{z}$ 


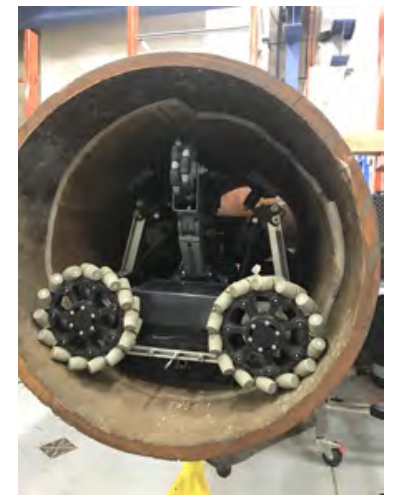

(a) NDT inspection robot front view.

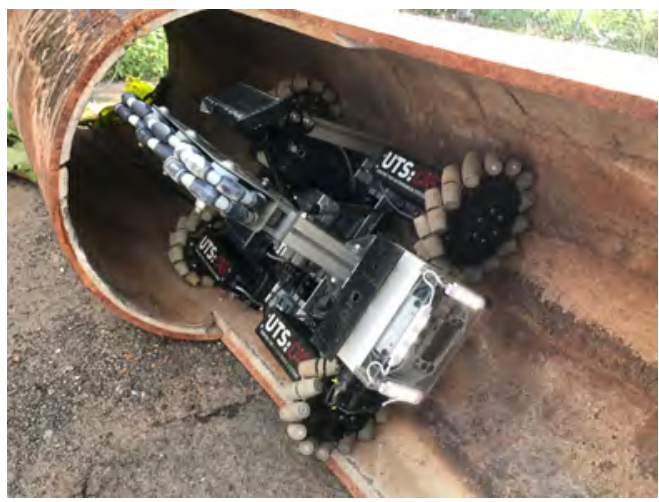

(b) NDT inspection robot in an open-cut pipe.

Figure 11: The NDT inspection robot (right figure depitcs the configuration with only one sensing arm mounted on).

axis. Driving each pair of diagonally opposite wheels with a single motor would achieve this requirement, however, the required drivetrain is complex and in the proposed designed control of each separate motors is implemented in software, as discussed below as part of the system overview.

To maintain stability during circumferential rotations, a set of free-wheeling omni-wheels are mounted on a parallel four bar linkage shown in Fig. 10a. This is linked to a pair of gas struts to provide a consistent opposing force to the mecanum wheels surface contact point. The applied force cancels out the gravity vector as the robot rotates, allowing consistent continuous surface contact for each of the wheels rollers. To achieve this the assembly is pressed against the pipe wall with a preload of approximately twice the robot weight, maintaining control authority regardless of orientation while simultaneously compensating for variation in pipe diameter. A linear actuator is included to retract the omni-wheels from the pipe surface during insertion. This actuator features a spline so that it does not affect the self correcting behaviour of the parallel linkage during normal operation. Fig. 10b demonstrates the applied force during the robots rotation along the vector F_act.

The PEC sensors are coupled to actuated lever arms using a stiff rubber joint. This joint allows the sensor to conform to the pipe surface in the presence of minor irregularities while maintaining a precise placement. The actuators drive until a stall condition is detected, allowing the sensor to be reliably placed on the pipe surface regardless of pipe variations or actuator drift.

Fig. 11 depicts the final robot in more detail in a pipeline in the laboratory (left), and in an open-cut pipeline for easier viewing in the field. 


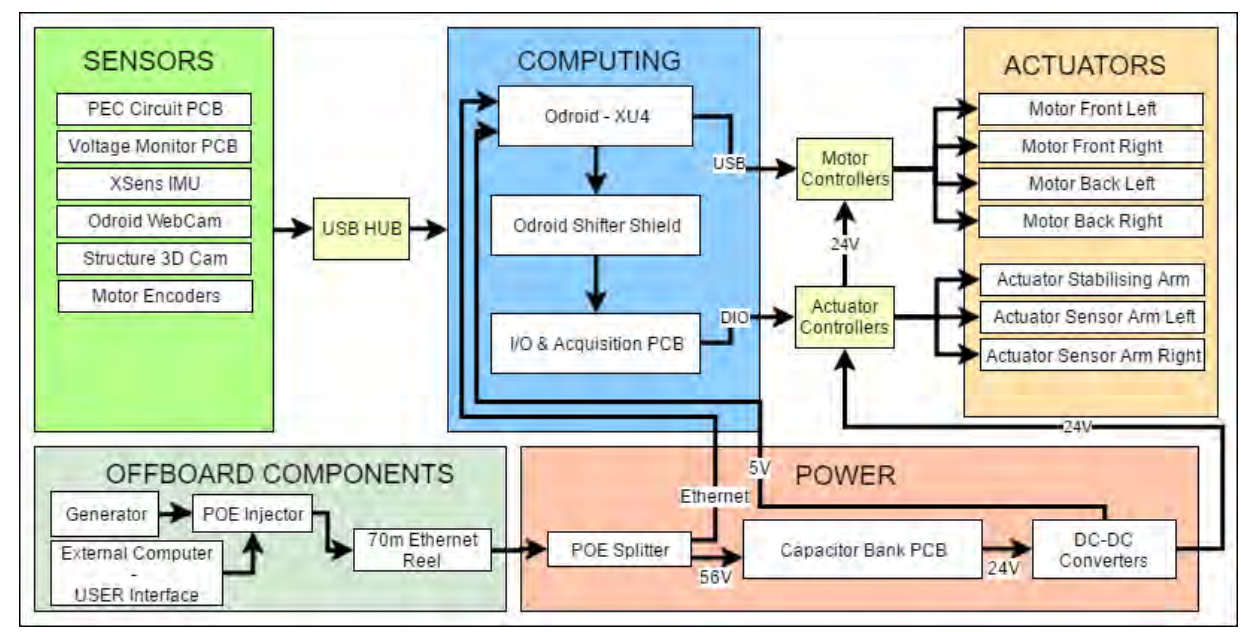

Figure 12: System block diagram depicting the four on-board major components on the robotic system (power, sensors, computing and actuators), and the off-board components linking the robot to a user-driven computer via a $70 \mathrm{~m}$ powerover-ethernet tether.

Table 1: Core Component specifications.

\begin{tabular}{ll}
\hline COMPUTING & Odroid XU4 - Arm based single board computer \\
SENSORS & Xsens Mti-10 IMU w/ Gyro 450 $/ \mathrm{s}$, acc $50 \mathrm{~m} / \mathrm{s}^{2}$ \\
& Odroid USB-Cam 30FPS, FOV: 68 \\
& 3D Structure Sensor w/ HFOV: $58^{\circ}, \mathrm{VFOV}: 45^{\circ}$ \\
CONTROL & Maxon Motor DCX26L, gear ratio 231:1, sensor 500 counts/turn \\
& Sensor Actuator - linear actuator, max force: $1000 \mathrm{~N}$ \\
& Stability Actuator - linear actuator, max force: $2500 \mathrm{~N}$ \\
POWER & PoE injector/splitter, 60W, 70m cable reel \\
\hline
\end{tabular}

\subsection{System Overview}

The system uses two computers, one on-board the robot for data acquisition and actuator control, and one outside the pipe for the user interface. The entire system runs from a generator on the surface with power delivered to the robot with a power over ethernet $(\mathrm{PoE})$ connection. The user interface can receive data and issue control commands back to the operator in real-time over the local area network (LAN) connection provided by the same ethernet tether. Fig. 12 demonstrates the overall hardware system layout whilst Table 1 list details about each component.

The long deployment duration precludes battery operation hence the choice of tethered PoE to provides for significantly longer operation times. The POE injector provides $60 \mathrm{~W}$ of power, the maximum supplied by readily available off-the-shelf equipment, so an ultracapacitor bank and bespoke charger was developed to supply bursts of high power while ensuring that the PoE equipment maintains an optimal power delivery rate. While deploying the sensors and taking a reading the average system power is $40 \mathrm{~W}$. This increases dramatically to $100 \mathrm{~W}$ when the motors are driven to reposition the robot. Since the time spent driving the motors is relatively low in comparison to sensing acquisition the 


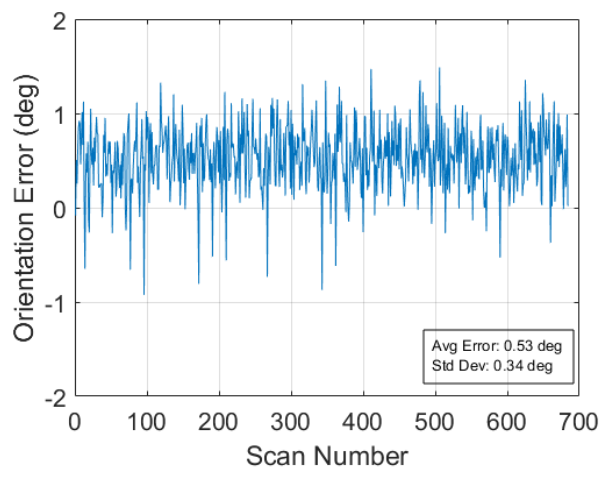

(a) Circumferential repeatability.

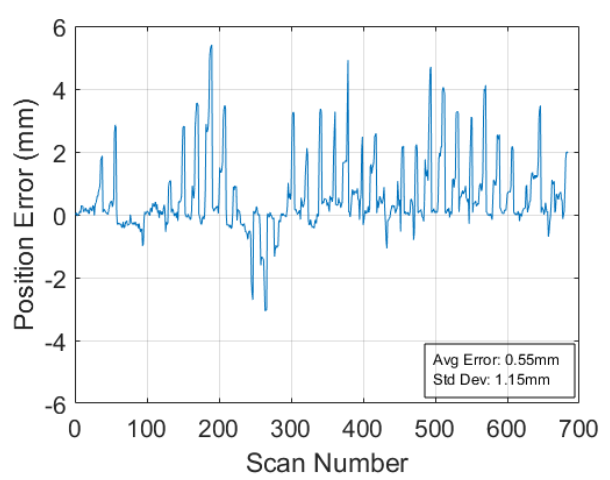

(c) Longitudinal error.

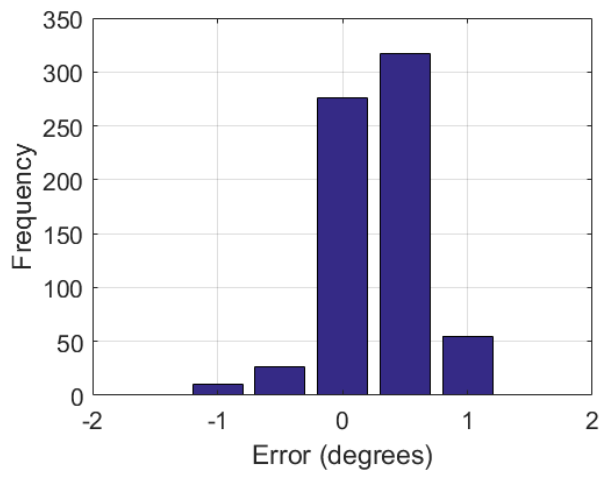

(b) Circumferential error histogram.

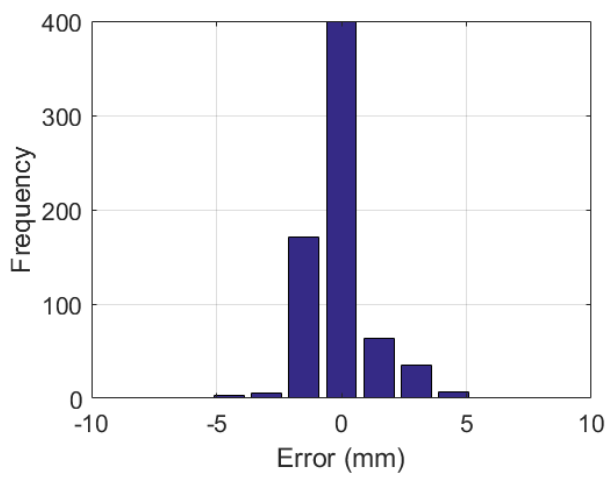

(d) Longitudinal error histogram.

Figure 13: Circumferential and longitudinal consistency. Circumferential deviations (top graphs) represent orientation errors of repeated commanded $10^{\circ}$ increment motions, as measured by an on-board IMU, whilst longitudinal errors shows the axial drift during these motions as measured by an external LIDAR (see Fig. 8b)

overall average power requirement is less than the $60 \mathrm{w}$ supplied by the PoE system. Thus, the chosen setup provides a steady power supply for the overall system on the condition that high power maneuvers are not sustained for extended periods, as is the case for the inspection of critical water mains which lay flat and straight in the ground.

The on-board Odroid, running Linux and the Robotic Operating System (ROS), receives data from the sensor suite through a powered USB hub and controls on-board actuators via digital input/outputs pins. Each sensor has it's own monitoring node to manage incoming data and publish to the communication layer. Custom task allocation/behaviour nodes then subscribe to the data streams, processing and publishing control commands as required to the motor and actuator nodes. System control is accomplished using a state machine, which allows both user and autonomous control modes for consistent data retrieval and safe user override. When switched into automatic scanning mode, the circumferential angle and longitudinal position are managed using independent set-point control loops. This simplifies both the kinematics and the algorithms required for control. Controlling circumferential angle is achieved using the on-board IMU and a standard PID control algorithm. The IMU publishes attitude data to ROS at a fixed rate of $100 \mathrm{~Hz}$. 
As each data packet is received, the attitude data is transformed into the local coordinate frame to maintain consistency even when the pipe is not levelled. Similarly, longitudinal control is achieved using odometry calculated using encoder readings published at a $100 \mathrm{~Hz}$ and filtered to detect wheel stalls. In addition, an overriding human in the loop (HITL) input allows direct control of the longitudinal position. This is used to recover when odometry fails due to motor stalls or excessive wheel slip during the ring-to-ring transitions. A laser distance sensor is utilised to confirm longitudinal position when conditionas are safe to deploy in the excavation pits.

\subsection{Motion Validation}

The circumferential and longitudinal consistency during automated scanning was verified at onsite trials, with angular repeatability and translational slip during rotation being the key metrics providing confidence in the sensor placement accuracy. Data was collected from an IMU to validate robot orientation, and a laser distance sensor was used to confirm longitudinal positions. Fig. 13a shows the measured rotation angle error during repeated scanning cycles of $180^{\circ}$ using $10^{\circ}$ increments. The average offset error of $0.53^{\circ}$ in relation to the set-point was found to be originated by the pressing action of the sensor against the pipe wall. Fig 13c shows longitudinal drift during these scans. An average error of $0.55 \mathrm{~mm}$ was produced with the maximum recorded error being $5.4 \mathrm{~mm}$. Since the PEC sensing method produces a result averaged over a 50x50mm area and the scan rings are spaced at increments of $100 \mathrm{~mm}$, a $5 \mathrm{~mm}$ drift in the longitudinal direction is deemed an acceptable inaccuracy well within the safety margins generally assumed for failure prediction analysis in civil infrastructures.

Given the overall sensing and mechanical constraints the system operates at slow speeds: runtime metrics measured during these tests showed an average spool completion time of around 166 minutes, or $1.3 \mathrm{~m} / \mathrm{h}$. This includes 216 seconds of automated scanning for each ring, with 30 to 60 seconds dedicated to motion from one ring to the next.

\section{Gaussian Processes for Spatial Regression Mapping from Sampled NDT Inspection Data}

As mentioned in Section 1 the salient novelty of the proposed robotic integrity assessment is the ability to carry out internal detailed inspections that enable dense mapping where identification of the geometry of wall loss patches can be confirmed. To achive this outcome various inspection patterns were studied to mitigate the slow robot examination speed reported in Section 3. A number of sampling strategies were experimented on and were extensively evaluated through cross validation using twelve thickness maps collected from exhumed pipelines during field trials and ground- 
truthed as decribed earlier in Section 2.2. This was carried out to determine the best inspection strategy that would minimise information losses against the original 2.5D wall thickness maps, given the nature of the thickness map data collected (Shi and Valls Miro, 2017). In this Section the proposed strategy undertaken to regress the missing data with the aid of a tailored Gaussian Process is presented.

Modeling spatial dependencies for 2.5D data has been largely studied in the past. Mathematically the problem can be described as a random field which is a collection of random variables of the form $\left\{y_{\mathbf{x}}, \mathbf{x} \in \mathbf{R}^{d}\right\}$, where $y_{\mathbf{x}}$ is the quantity measured at the position $\mathbf{x}$ (Lord et al., 2014). Random fields are also known as spatial processes, for instance, univariate (Kroese and Botev, 2015) or multivariate (Schlather et al., 2015) processes, that are defined for modeling spatially arranged measurements and patterns. Random fields can be statistically specified by mean and covariance (Lord et al., 2014; Kroese and Botev, 2015). When the mean is a constant, depending on the covariances there are stationary random fields whose covariances are invariant under translations, isotropic stationary random fields whose covariances are invariant under both translations and rotations, and anisotropic stationary random fields whose covariances are directionally dependent (Lord et al., 2014; Kroese and Botev, 2015). A more specific type of random field being studied extensively is Gaussian Random Fields (Davies and Bryant, 2013), which is also known as Gaussian Spatial Processes (Kroese and Botev, 2015) or Gaussian Processs (GPs) (Rasmussen and Williams, 2006; Bishop, 2006).

In robotics, GPs have been employed in terrain and surface modeling (O'Callaghan and Ramos, 2012; Smith et al., 2010; Vasudevan et al., 2009). In all these works, the utilization varies with the properties of the applications, the structure of the model and the usage of the correlation information. The use of these probabilistic tools for thickness mapping and fusion has recently been tackled by the authors for an application on pipeline condition assessment (Sun et al., 2015; Vidal-Calleja et al., 2014).

\subsection{Gaussian Processes}

GPs define the probability distribution over functions, any finite number of which have consistent joint Gaussian distribution. Consider $n$ thickness-location pairs $\mathscr{D}$ defined as:

$$
\mathscr{D}=\left\{\left(y_{1}, \mathbf{x}_{1}\right),\left(y_{2}, \mathbf{x}_{2}\right), \ldots,\left(y_{n}, \mathbf{x}_{n}\right)\right\}
$$


where $\mathbf{x}_{i} \in X$ is the position in $\mathbf{R}^{d}$ ( $d=2$ in the case of $2.5 \mathrm{D}$ data) where the thickness measurements $y_{i} \in Y$ was taken. The data set $\mathscr{D}$ is assumed to be drawn from a noisy process

$$
y_{i}=f\left(\mathbf{x}_{i}\right)+\varepsilon_{i}, \text { where } \varepsilon_{i} \sim \mathscr{N}\left(0, \sigma_{n}^{2}\right),
$$

where noise $\varepsilon_{i}$ follows independent, identically distributed zero-mean Gaussian with variance $\sigma_{n}^{2}$. GPs are used to learn the distribution $p(f \mid X, \mathscr{D})$ from $\mathscr{D}$ and have the capability of inferring $p\left(f \mid X^{*}, \mathscr{D}\right)$ for arbitrary location $X^{*}$.

Having specified the mean and covariance functions ${ }^{1}$ and identified the hyper-parameter set $\theta$, parameter estimation can be conducted through optimization by maximizing the likelihood function as described in equation 4 .

$$
\begin{array}{r}
\log p(\mathbf{y} \mid X)=-\frac{1}{2}(\mathbf{y}-m(X))^{\top} K_{y}^{-1}(\mathbf{y}-m(X)) \\
-\frac{1}{2} \log \left|K_{y}\right|-\frac{n}{2} \log 2 \pi
\end{array}
$$

where $m$ and $K$ are the mean and covariance functions respectively, and $K_{y}=K(X, X)+\sigma_{n}^{2} I$ denotes the joint prior distribution covariance of the function at positions $X$. The variance of the noise $\sigma_{n}^{2}$ constitutes another parameter to be learned together with $\theta$.

Inference at a finite set of query locations $X^{*}$ can be performed by calculating the predicted mean $\mu_{P}$ and covariance $\Sigma_{P}:$

$$
\begin{gathered}
\mu_{P}=m\left(X^{*}\right)+K\left(X^{*}, X\right) K_{y}^{-1}(\mathbf{y}-m(X)) \\
\Sigma_{P}=K\left(X^{*}, X^{*}\right)-K\left(X^{*}, X\right) K_{y}^{-1} K\left(X^{*}, X\right)^{\top}
\end{gathered}
$$

The covariance matrix $K\left(X^{*}, X\right)$, obtained from a given covariance function $K$, is indicative of the cross-correlation between the function at $X^{*}$ and the training inputs $X$.

\subsection{Covariance Function Design}

GPs are thus completely specified by the choice of mean and covariance functions. The mean function can be usually set to be a constant value, whilst the covariance function controls the smoothness of the process, and its parameters govern the effective range of correlation and the variability observed in the data. There is no single covariance function that fits all modeling tasks. Depending on the purpose at hand and any insights that might be available from

\footnotetext{
${ }^{1}$ The terms covariance and kernel function are used indistinctively.
} 
the underlying physical phenomenon described by the data, modified or composite covariance functions may allow more flexibility in the model. Indeed, the usage of prior knowledge in choosing appropriate covariance functions is encouraged in the literature (Tesch et al., 2011), e.g. using periodic covariance functions in the analysis of seasonal variation and physical phenomena (Rasmussen and Williams, 2006) (Tartakovsky and Xiu, 2006).

In effectively modelling the wall thickness of buried pipelines a number of covariance functions were considered which included characteristics related to the physical properties of the target to be modelled. Three commonly used stationary kernel functions (detailed in the next Section) with additional characteristics revealed by the data in terms of directionality and periodicity were tested. These were incorporated in the form of a 2D anisotropic composite covariance function with a periodical wrapping construction. The period in the circumferential direction was clamped to guarantee the $2 \pi$ periodic property of a pipe wall thickness map. An example with a $2 \mathrm{D}$ Matern kernel $(\mathrm{v}=3 / 2)$ is shown in equation 7

$$
K\left(X, X^{*}\right)=K(r)=(1+\sqrt{3} r) \exp (-\sqrt{3} r)
$$

where the input distance $r$ is defined by

$$
r=\frac{1}{l} \sqrt{\left(X-X^{*}\right)^{T}\left(X-X^{*}\right)}
$$

for an isotropic kernel, with $l$ being the length-scale, and by

$$
r=\sqrt{\left(X-X^{*}\right)^{T} \wedge^{-2}\left(X-X^{*}\right)}
$$

for an anisotropic kernel, where $\wedge$ is a diagonal matrix with characteristic length-scales $l_{1}$ and $l_{2}$ on the main diagonal.

In the case of modelling periodical data, an established approach is warping. This is generally done by mapping each one-dimensional input variable $x$ to two-dimensional input variable

$$
\mathbf{u}(x)=\left[\sin \left(x_{p}\right), \cos \left(x_{p}\right)\right]
$$

where $x_{p}=2 \pi x_{p}$, and $p$ is the period parameter, hence constructing a covariance matrix $K\left(\mathbf{u}(X), \mathbf{u}\left(X^{*}\right)\right)$ to turn an anisotropic kernel periodic (Rasmussen and Williams, 2006). 


\subsection{Model Selection}

Model selection was carried out by using the ground-truth dataset refered to in Section 4, composed of twelve 3D laser thickness maps attained from exhumed pipeline spools from the same pipeline, so that the original 2.5D wall thickness maps could be recovered via the proposed GP regressor from the in-field sample data collected by the NDT robot.

The proposed covariance function was selected by considering the physical properties of the target to be modelled described in the preceding Section, and by means of comparing the Akaike information criterion (AIC) of each model. AIC is a relative metric between potential models defined as

$$
A I C=2 k-2 \ln (L)
$$

where $k$ is the number of model parameters, and $L$ is the maximum value of the likelihood function for the model in parameter estimation (Burnham and Anderson, 2002). While the Log Marginal Likelihood (LML) is also often used in the literature for this purpose, for models with relatively low complexity - as those proposed in this work - the values of both LML and AIC are comparable, so AIC was selected as arguably it provide a slight advantage over LML in also penalising models with additional complexity for similar fitting, thus discouraging overfitting

Three commonly used kernel functions with different setups were tested to establish the guidance on selecting the most appropriate model. The basic characteristics of the three candidate kernel functions in a simplified isotropic 1D scenario are illustrated in Fig. 14a, given the difficulty in visualising a 2D anisotropic kernel. Table 14b shows that under the same setup, both anisotropic and periodic composite covariance model setups produce lower AIC given the characteristics of the pipeline data. The combination of these two add-ons gives the best performance for cylindrical structures such as buried pipes, which corresponds to the observation that pipe wall thickness correlations in the extracted data appear differently in circumferential and axial directions, and the correlation in circumferential direction is $2 \pi$ periodic. Among these kernel functions, Matern v3/2 always produced the lowest AIC under the same setup. Higher order Matern are generally indistinctive from the Square Exponential case, whilst given the apparent trend that less smooth kernels perform better, it would be reasonable to also consider the lowest order Matern v1/2. However, these models become quite "rough" and while functions are continuous they loose the differentiablily, a characteristic that was not readily apparent in the data so was not perceived as a fitting alternative. Therefore, Matern v3/2 with anisotropic and periodic setup was selected as the most appropriate kernel for modeling pipe wall thickness maps.

It is worth noting that reconstruction error was also experimented upon as a model selection metric, and statistical 


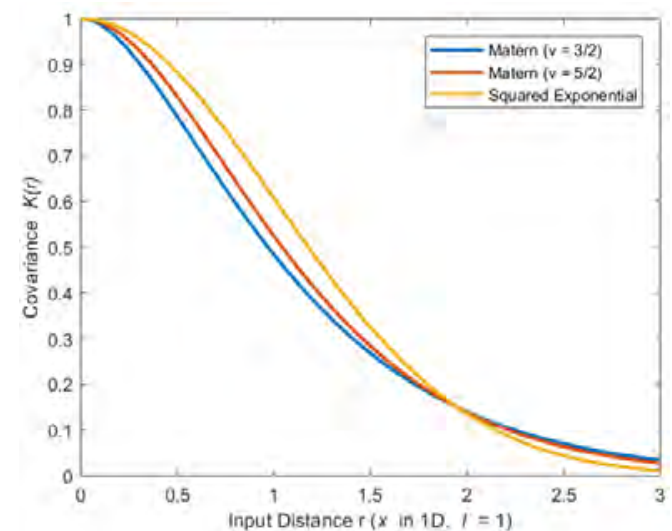

\begin{tabular}{|l|c|c|c|c|c|}
\hline Kernel & $\begin{array}{c}\text { Anisotropic } \\
\text { Add-on }\end{array}$ & $\begin{array}{c}\text { Periodic } \\
\text { Add-on }\end{array}$ & \#Params. & $\begin{array}{c}\text { AIC } \\
(\text { mean })\end{array}$ & $\begin{array}{c}\text { AIC } \\
(\mathrm{std})\end{array}$ \\
\hline Matern $(\mathrm{v}=3 / 2)$ & $\mathrm{N}$ & $\mathrm{N}$ & 4 & 2448 & 1475 \\
\hline Matern $(\mathrm{v}=5 / 2)$ & $\mathrm{N}$ & $\mathrm{N}$ & 4 & 2718 & 1434 \\
\hline Sq. Exp. & $\mathrm{N}$ & $\mathrm{N}$ & 4 & 3531 & 1390 \\
\hline Matern $(\mathrm{v}=3 / 2)$ & $\mathrm{Y}$ & $\mathrm{N}$ & 4 & 2001 & 1411 \\
\hline Matern $(\mathrm{v}=5 / 2)$ & $\mathrm{Y}$ & $\mathrm{N}$ & 4 & 2283 & 1377 \\
\hline Sq. Exp. & $\mathrm{Y}$ & $\mathrm{N}$ & 4 & 3034 & 1415 \\
\hline Matern $(\mathrm{v}=3 / 2)$ & $\mathrm{Y}$ & $\mathrm{Y}$ & 7 & 1922 & 1423 \\
\hline Matern $(\mathrm{v}=5 / 2)$ & $\mathrm{Y}$ & $\mathrm{Y}$ & 7 & 2147 & 1388 \\
\hline Sq. Exp. & $\mathrm{Y}$ & $\mathrm{Y}$ & 7 & 3051 & 1473 \\
\hline
\end{tabular}

(a) Characteristics of the 3 candidate covariance functions (b) Combined kernel models studied with anisotropic and periodic add-ons to a given tested, graphically depicting the statistical relationship be- standalone covariance function, and AIC model selection metric. tween any two points " $r$ " distance appart in 1D.

Figure 14: Gaussian Process kernel model selection. The combined anisotropic and periodic covariance functions were tested on ground truth data thickness maps from a large number of exhumed pipeline spools, and their performance in terms of AIC metric used to select the kernel to regress the data colllected with the NDT inspection robot (low AIC values are indicative of a better fitting).

tests (ANOVA) computed on sample mean and variances. It was found that Matern kernels (tested with different hyper-parameters) behaved statistically the same in terms of the reconstruction error. On the other hand, given the dense training data around missing data, Squared Exponential produced statistically significantly worse results than any Matern kernel.

\subsection{Stability of the Model Selection}

The stability of the AIC model section metric was estimated through bootstrapping (Efron and Tibshirani, 1994) training samples from the twelve GT thickness maps. The theoretical foundation of bootstrap resampling allows

Table 2: Test-bed specifications, adapted from (Valls Miro et al., 2014).

\begin{tabular}{ll}
\hline Location & Sydney, NSW, Australia \\
Year Installed & 1922 \\
Nominal Pipe Diameter & $600 \mathrm{~mm}$ \\
Internal Pipe Diameter & $579 \mathrm{~mm}$ to $590 \mathrm{~mm}$ (with cement lining) \\
External Pipe Diameter & $662 \mathrm{~mm}$ to $666 \mathrm{~mm}$ \\
Nominal Wall Thickness & Approx. $27 \mathrm{~mm}$ \\
Material & Pit Cast Iron \\
Internal Liner & Cement (installed in-situ in 1964) \\
Cement Lining Thickness & $9.5 \mathrm{~mm}$ to $16.5 \mathrm{~mm}$ \\
Generic Spool Length & Approx. $3.6 \mathrm{~m}$ \\
Jointing & Lead run joints (tar soaked hemp sealants) \\
Total length used for research & Approx. $1 \mathrm{~km}$ \\
\hline
\end{tabular}




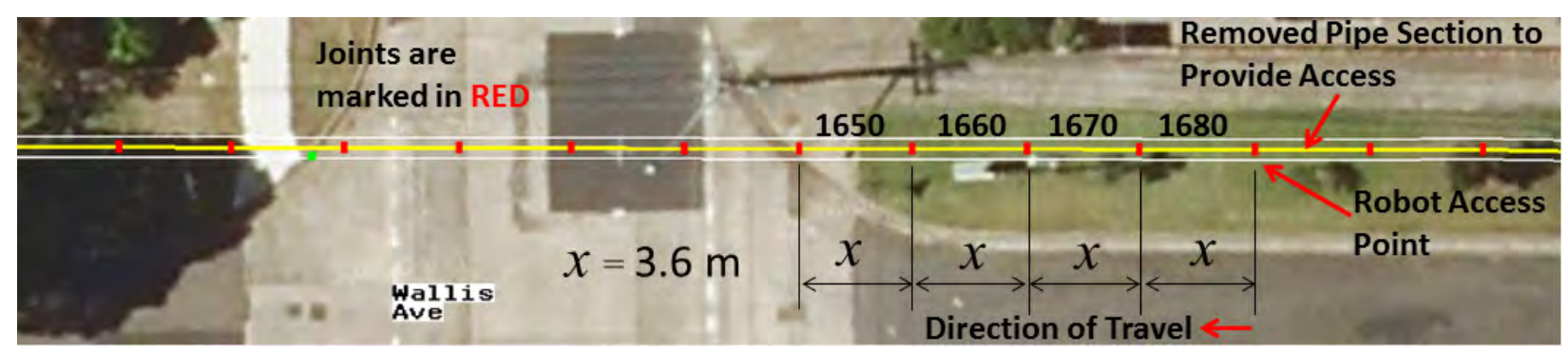

Figure 15: A typical inspection plan supplied to the utility parner for the deployment of the NDT inspection robot.

estimating the distribution of a statistic using random sampling methods. Specifically in this evaluation, training map samples were randomly drawn with replacement for 100 iterations to feed in AIC parameter estimation and characterize the candidate model. The last two columns in Table 14b collects the results, which indicate that all candidate kernels have similar AIC variances yet the propose anisotropic periodic Matern $(v=3 / 2)$ kernel exibits the lowest AIC mean.

\section{$5 \quad$ Field Pipeline Inspection Results}

The proposed robotic device has been extensively deployed in a buried $1 \mathrm{~km}$ live CI Cement Lined (CICL) pipeline provided by a utility in Sydney, Australia, in what effectively constitutes a unique worldwide opportunity for the advancement of NDT sensing and automation research in the field (Valls Miro et al., 2014). The pipeline has been decommissioned and is therefore no longer part of the utility's live network. However a connection point to an adjacent $600 \mathrm{~mm}$ water main and various scour valves and hydrants allow for the pipeline to be pressurised and discharged as needed. Details of the pipeline are collected in Table 2. Pipe sections between 3 and $4 \mathrm{~m}$ in length were targetted for scanning by inserting the inspection robot through a removed pipe section, be that a previously replaced section, as shown in Fig. 1b, or a new cut-out. An example of an inspection plan is shown in Fig 15.

Robot localisation with respect to an entry point while travelling towards a section targetted for inspection was done by means of robot odometry, measurement of tether release and accounting for spool joints traversed as seen by the robot camera. Validation from an external laser scanner mounted at the entry point as seen inFig. $8 \mathrm{~b}$ was also used when it was deemed safe to be deployed in the field excavation pit, and there was line of sight within the laser range. Moreover, discontinuity on spool joints also reveals a characteristic PEC signal comparable to a crack that was also exploited in case of ambiguity about spool length.

After reaching the target spool, circumferential and longitudinal ring inspections were undertaken as described in Section 3 to generate maps such as those depicted in Fig. 16. Following the inspection pattern ascertained in (Shi and 


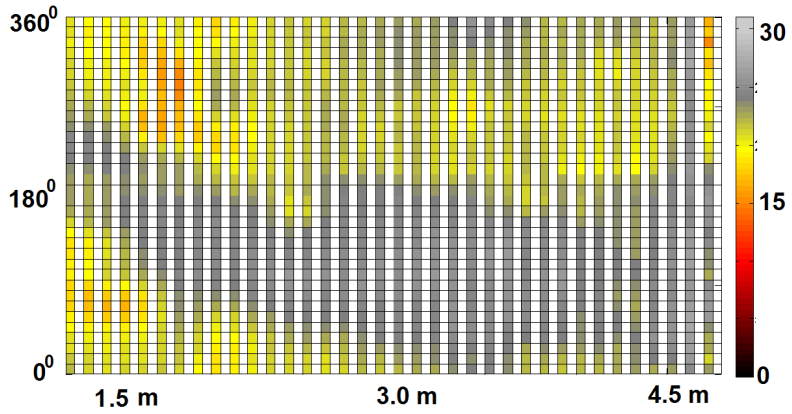

(a) Target 1680 in Fig. 15(plan) and 17b.

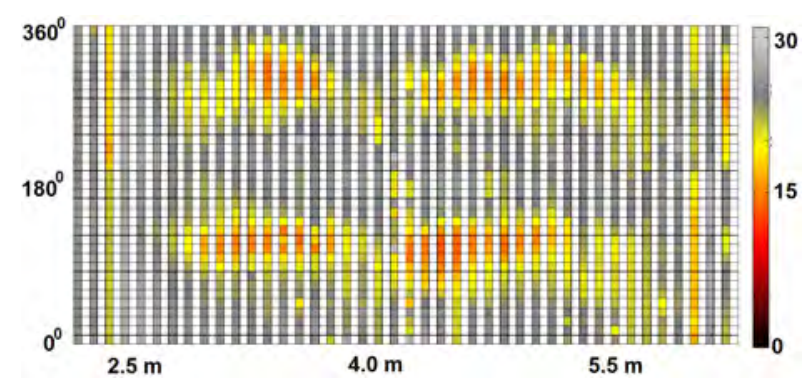

(c) Target 1060 in Fig. 17a.

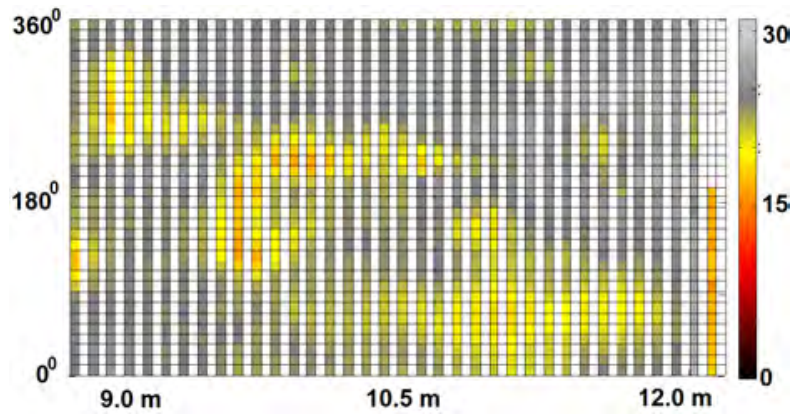

(b) Target 1670 in Fig. 15(plan) and 17b.

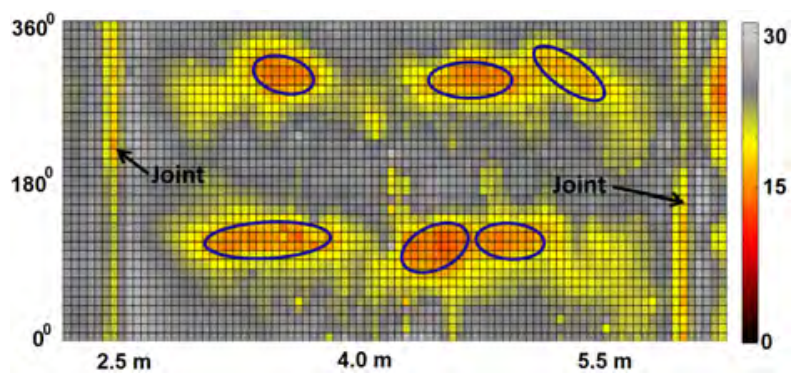

(d) GP-inferred map depicting joints and identified corrosion patches.

Figure 16: Various examples of remaining pipe wall thickness maps as measured by the robotic wall inspection during field deployment on the buried critical water main. Distances shown are with respect to the edge of closest access point.

Valls Miro, 2017), circumferential rings $100 \mathrm{~mm}$ appart in axial distance were evaluated with the robotic platform, which given the $50 \mathrm{~mm}$ sensor footprint effetively meant skipping every other ring with considerable time savings. Examples of these are shown in Fig 16a, 16b and 16c. Adopting the GP model selection described in Section 4, a model could be learned for each inspected spool and full inference on the missing rings undertaken. An example of the final outcome achieved is shown in Fig. 16d, where measurements indicative of the lead run joints are also shown. Where wall loss is present the spread of the reduction is clearly evident and can be identified and measured. Such patches are modelled as ellipsoids (also depicted in Fig. 16d overlaid over the reconstructed map), and their defining parameters can then be incorporated for stress calculation and remaining life prediction of the asset (Ji et al., 2017).

The final thickness maps gathered by the NDT robot during an extensive period of deployment between 2016 and 2018 are depicted in Fig. 17, referenced on an aerial picture showing the location where the pipeline is buried. The layout of the pipeline spool structure is also shown in yellow, where white lines are illustrative of the pipeilne diameter, and red segments identify spool joints, with the spool number inspected with the robot labelled accordingly. The date of inspection has also been added. Further details about the full program of inspections and various other tests undertaken with the NDT robot during the field deployment can be found in a comprehensive supplementary document associated to this manuscript that has been deposited in the journal webpage for the interested reader. 


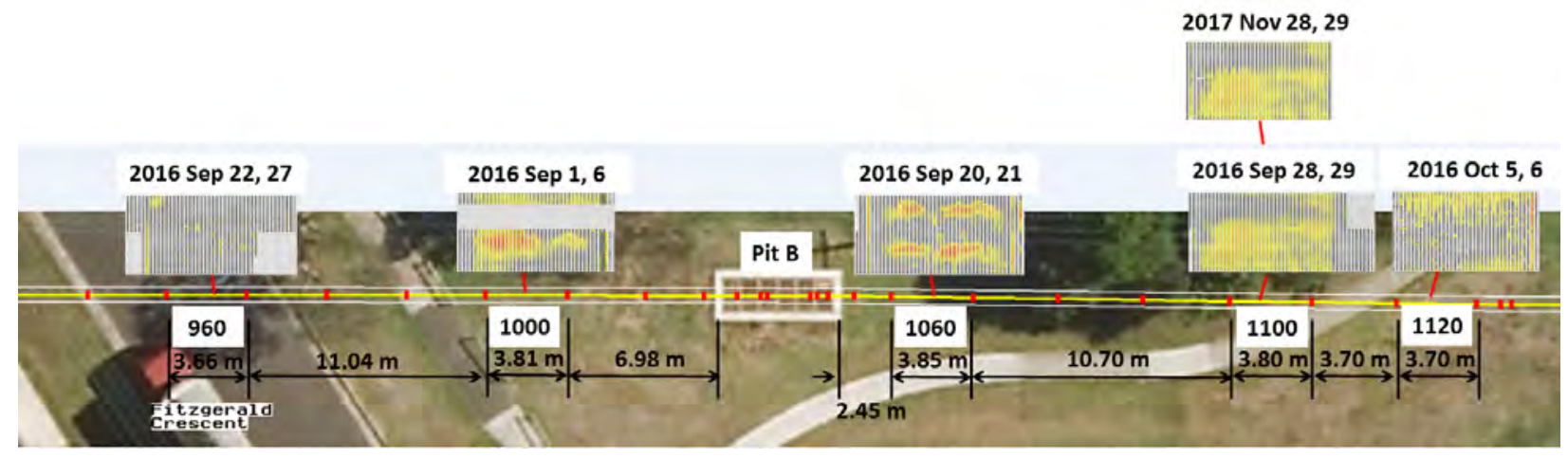

(a) Pits inspected from entry point "Pit B".

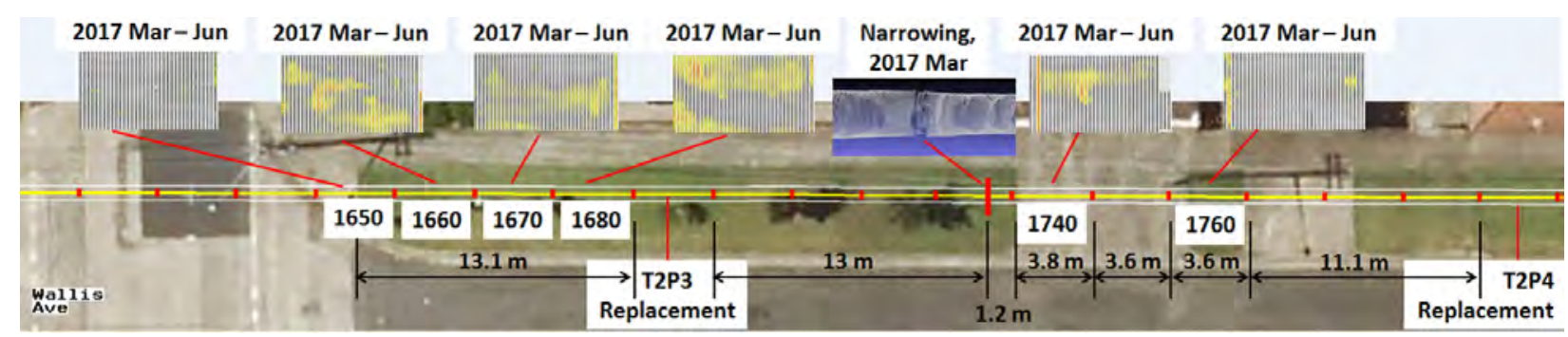

(b) Pits inspected from entry point "T2P3 Replacement".

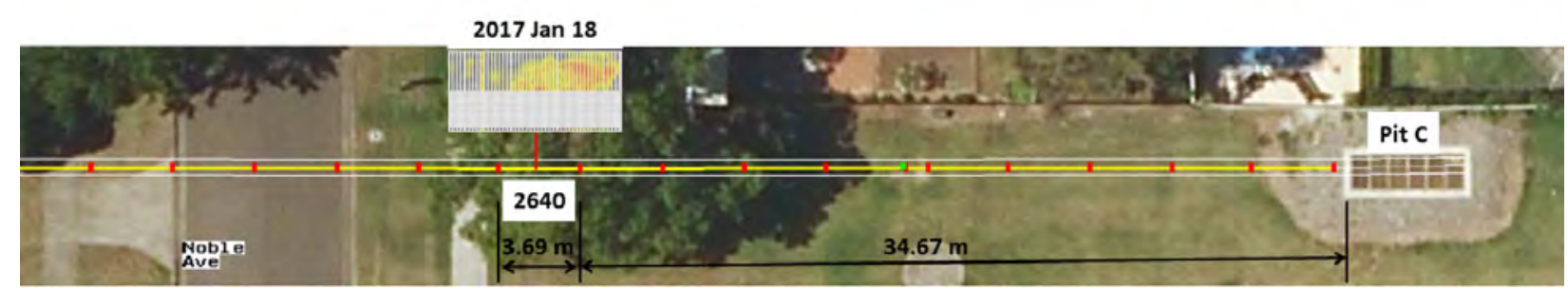

(c) Pits inspected from entry point "Pit C"..

Figure 17: Final 2.5D spool thickness maps attained from the field deployment of the NDT robotic inspection robot on a buried critical water main in Sydney during 2016-17-18. Three sections closer to the deployment entry points are shown. In the middle figure, two access points (T2P3 and T2P4) were required given the unexpected narrowing found in the pipeline (see Section 5.1).

Prior to using the robotic tool for extensive measurements, repeatability tests were also carried out on pipe sections at the test-bed to ascertain the performance of the robotic inspection unit in-situ. Results from one of the tests are shown in Fig. 18. The error histogram in Fig. 18c suggests a close to zero-mean Gaussian $(0.112 \mathrm{~mm}$ mean, $0.869 \mathrm{~mm}$ standard deviation). Information such as minimum, maximum and average thickness of the inspected pipe section are key parameters of interest to water utilities for stress analysis and asset management in general. Table 18d collects the most typical quantitative information being currently reported with the robotic device on the two inspections shown Map A (Fig. 18a) and Map B (Fig. 18b), corresponding to target 1100 in Fig. 17a. 


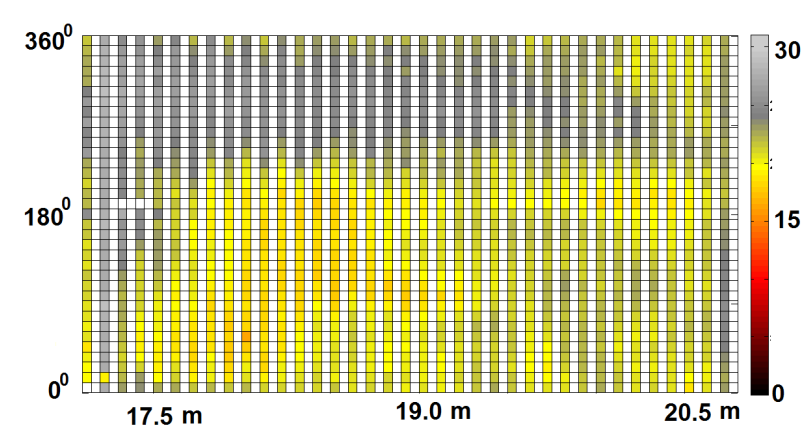

(a) Map A (Target 1100).

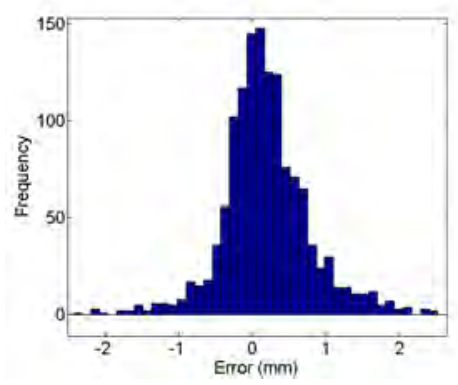

(c) Errors between maps.

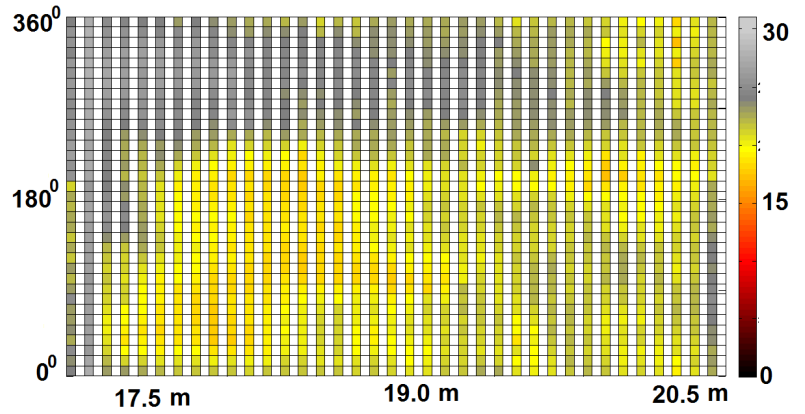

(b) Map B (Target 1100).

\begin{tabular}{lcc}
\hline & Map A & Map B \\
\hline Minimum thickness & 17.4 & 16.2 \\
Maximum thickness & 28.1 & 28.8 \\
Average thickness & 21.8 & 21.9 \\
\hline
\end{tabular}

(d) Inspection measurements (mm).

Figure 18: Robotic inspection repeatability tests on a single pipe section, target 1100 in Fig. 17a .

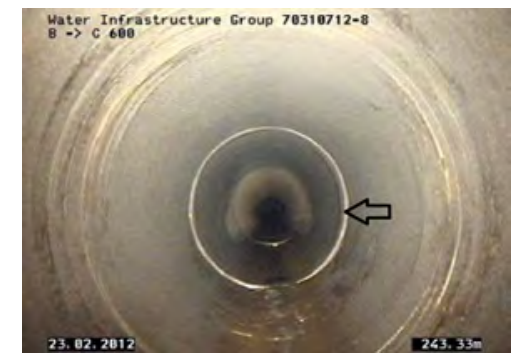

(a) Picture of anomalous narrowing (white ring at pointer).

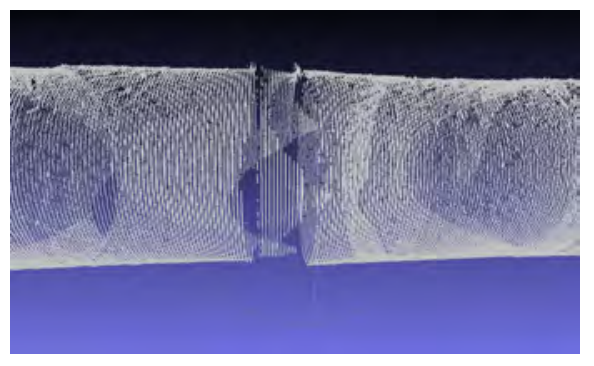

b) 3D reconstruction of anomaly captured by RGBD robot sensor.

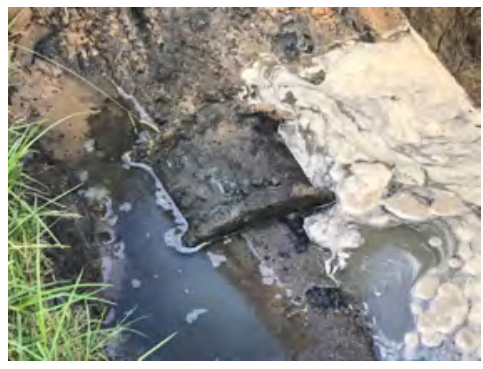

(c) Outer clamp found after excavation.

Figure 19: Unchartered pipe anomaly found during inspection; verification excavation.

\subsection{Pipe Inner Surface Profiling}

In addition to PEC measurements, perceptual information from video streaming and point clouds of the pipe inner surface (cement lining) can also be recorded with the RBG camera and the 3D structure sensor mounted at the front of the robot. The latter in particular allows mapping the geometry of the pipe inner surface in order to evaluate the surface unevenness, variation in the nominal pipe diameter and mapping the structure of in-pipe features (chainage, off-takes, valves). Moreover, reconstructing the inner surface profile has the advantage that it enables identifying and locating unchartered coarse anomalies present on the cement lining surface which may impede motion of ILI tools. 
An example of the latter was apparent during one of the inspections where the robot encountered an abnormality in the form of pipe narrowing during the experiment is shown in Fig. 19b. The mean diameter of the narrow region was observed to be $579 \mathrm{~mm}$ while the expected nominal diameter of the cement lined inner surface is expected to be close to $600 \mathrm{~mm}$. A posterior excavation by the utility found an unknown outer clamp as shown in Fig 19c, whose origin was investigated but not found on any records.

\section{Key Lessons Learned from the Field Deployment}

The operational aspects of interfering with a critical asset that affects large populations in their daily lives, such as the supply of drinking water, can not be understimated. The deployment of the unit on a critical pipeline was indeed a carefully orchestrated exercise. Likewise, the extraction and replacement of sections for careful examination and ground truthing was a significant undertaking in terms of logistics and resources (both personnel and monetary, including flow control, excavation, transportation, grit-blasting and pipe preservation). It was revealing the extent of consultations required with the many water utility teams that had to get involved during all phases of the project.

The actual field deployment of the robotic unit brought about some pivotal aspects to contend with, which in summary could be identified as: (1) Unexpected anomalies in the pipe in the form of surface unevenness (e.g. in the rendering of the cement lining), or pipe narrowings due to unknown features (e.g. an unchartered replaced pipe); (2) Boundaries in the robustness of the hardware, given the harsh deployment conditions; (3) Impact of the limited speed on the logistics surrounding the customer support and civil delivery team efforts to be able to deploy the robot ( $\sim 14$ hours to cover $50 \%$ of a $3.5 \mathrm{~m}$ long, $600 \mathrm{~mm}$ diameter pipe section); and the (4) Need to introduce a level of compliance in the sensor mountings to absorb the undocumented asymmetries and pipe manufacturing tolerances, so as to maintaining close contact with the pipe walls for NDT sensing. Specific details of various of the issues encountered in regards to the above are provided in the supplementary material document associated to this manuscript that has been deposited in the journal webpage for the interested reader.

Following the learnings drawn from entertaining these issues in the field, authors have begun investigating faster sensing architectures (multiple PEC sensors operating simultaneously) that can assess over $10 \mathrm{~m}$ of pipe length with $100 \%$ coverage (600 mm diameter) within an hour. Authors are also investigating new robot architectures which enable significant size adjustments on encountering anomalies such as reduction/enlargement in the pipe diameter during the inspection, or the presence (sometimes unexpectedly) of other pipe fittings and appurtenances.

Lastly, an interesting observation made during field deployment related to the actual choice of robot kinematic design, 
with one of the rear wheels of the robot experiencing signs of undue stress (both the left rear wheel assembly, as well as its driving motor, failed on two occasions). This was attributed to unforeseen misalignment or asymmetry in the implemented robot hardware, forcing the left rear side to experience accentuated tension. This was primarily a result of the mechanical robot design with an arm linkage to allow rotational mobility, as well as assured grip of the wheels againts the pipe walls during longitudinal motion. The lack of suspension meant that the applied forces when pressing the wheels against the wall were substantial, often inducing significant stress on the driving wheel actuators. Measures can be taken to minimize or diverge from the requirement of the whole robotic unit having to rotate by facilitating a sensing unit mechanism able to rotate while the rest of the robotic device does not, and such architectures are currently being investigated.

\section{Concluding Remarks}

An in-line robotic solution for the inspection of buried critical water mains and its evaluation during field deployments has been presented in this paper. A singular kinematic locomotion design that optimises mobility in such tubular environment has been coupled with an embedded NDT sensing solution based on PEC for measurements unsusceptible to sensor lift-off, as typically found in cement lined water pipelines. A tailored covariance function suitable for pipeline geometries has also been studied in a Gaussian Processes framework to regress missing sensor data.

The data gathered represents not only a visual understanding of the condition of the pipe for asset managers, but also constitutes a quantative input to a remaining-life calculation that defines the likelihood of the pipeline for future renewal or repair. The device addresses a utility sector need for an automatic NDT inspection vehicle that can report dense pipe wall thickness discrimination as prescribed by failure prediction analysis, and that can be deployed in an opportunistic manner - e.g. when a mains break occurs, or during valve inspection or repair programs when pipelines are discharged and access made available. Extensive results have proven the validity of the solution on laboratory tests and field pipeline inspections which demonstrate the feasibility of the device and sensing configuration to provide meaningful 2.5D geometric maps. Future work is set on further characterisation and inclussion of sensor measurement uncertainties in the analysis of the pipeline remaining-life calculations.

As highlighted in Section 6, following the learnings presented in this paper a new version of the robot is currently under development with alternative mobility and sensor arrangement to facilitate faster robotic deployment, as well as faster signal processing and acquisition. As part of that development, consideration is also being given to the unsolved problem of relating complex pipe wall geometry to a PEC signal, a somewhat unorthodox sensor in the space of probabilistic mapping. For practical purposes the NDT sensor measurements used in this work have been approximated 
to the average thickness under the footprint of the sensor (a $50 \mathrm{~mm}$ x $50 \mathrm{~mm}$ region) with reassuring results, although in reality the sensor's measurement generalises to a domain. Due to the unsolved problem of accurately relating complex geometry to the signal, it is not trivial to assume or define a precise sensor model, and a compatible kernel to capture such behaviours for regression, a task that requires further investigation.

\section{Acknowledgement}

This work is an outcome from the Advanced Condition Assessment and Pipes Failure Prediction project (www. criticalpipes.com), an international R\&D consortium funded by Sydney Water Corporation, Water Research Foundation (USA), Melbourne Water, Water Corporation (WA), UK Water Industry Research, South Australia Water Corporation, South East Water, Hunter Water Corporation, City West Water, Yarra Valley Water, Queensland Urban Utilities, University of Technology Sydney, Monash University and University of Newcastle.

This manuscript represents an extension to (Valls Miro et al., 2017) where additional details on the PEC sensing and robot system design have been documented, alongside more detailed results of the field deployment and discussions fom those. Moreover, an in-depth description of a novel kernel covariance function and the GP-based algorithm employed to generate 2.5D thickness maps from the field data have also been added.

\section{References}

Bishop, C. M. (2006). Pattern recognition and machine learning, chapter Kernel Methods, pages 291-324. Information Science and Statistics. Springer-Verlag, New York.

Burnham, K. P. and Anderson, D. R. (2002). Model selection and multimodel inference: a practical informationtheoretic approach, chapter Information and Likelihood Theory: A Basis for Model Selection and Inference, pages 60-64. Springer-Verlag, New York.

Davies, T. M. and Bryant, D. (2013). On circulant embedding for Gaussian random fields in R. Journal of Statistical Software, 55(9):1-21.

Efron, B. and Tibshirani, R. J. (1994). An introduction to the bootstrap, chapter Information and Likelihood Theory: A Basis for Model Selection and Inference, pages 60-64. Chapman \& Hall/CRC, New York.

Fu, F. and Bowler, J. (2006). Transient eddy-current driver pickup probe response due to a conductive plate. IEEE Transactions on Magnetics, 42(8):2029-2037. 
Huang, C. and Wu, X. (2015). An improved ferromagnetic material pulsed eddy current testing signal processing method based on numerical cumulative integration. NDT \& E International, 69:35-39.

Huang, C., Wu, X., Xu, Z., and Kang, Y. (2011). Ferromagnetic material pulsed eddy current testing signal modeling by equivalent multiple-coil-coupling approach. NDT \& E International, 44(2):163-168.

Huang, C., Xinjun, W., Zhiyuan, X., and Kang, Y. (2010). Pulsed eddy current signal processing method for signal denoising in ferromagnetic plate testing. NDT \& E International, 43(7):648-653.

Ji, J., Robert, D., Zhang, C., Zhang, D., and Kodikara, J. (2017). Probabilistic physical modelling of corroded cast iron pipes for lifetime prediction. Structural Safery, 64:62-75.

Kodikara, J., Valls Miro, J., and Melchers, R. (2016). Failure prediction of critical cast iron pipes. Advances in Water Research, 26(3):6-11.

Kroese, D. P. and Botev, Z. I. (2015). Spatial process simulation. Stochastic Geometry, Spatial Statistics and Random Fields. Lecture Notes in Mathematics, vol. 2120. Springer, Cham - Switzerland.

Liu, Z. and Kleiner, Y. (2013). State of the art review of inspection technologies for condition assessment of water pipes. Measurement, 46(1):1-15.

Lord, G. J., Powell, C. E., and Shardlow, T. (2014). An introduction to computational stochastic PDEs, chapter Random Fields, pages 257-310. Cambridge University Press.

O'Callaghan, S. and Ramos, F. (2012). Gaussian process occupancy maps. International Journal of Robotic Research, 31(1):42-62.

Rasmussen, C. E. and Williams, C. K. I. (2006). Gaussian process for machine learning, chapter Regression, pages 7-32. MIT press, Cambridge.

Schlather, M., Malinowski, A., Menck, P. J., Oesting, M., and Strokorb, K. (2015). Analysis, simulation and prediction of multivariate random fields with package randomfields. Journal of Statistical Software, 63(8):1-25.

Shi, L. and Valls Miro, J. (2017). Towards optimised and reconstructable sampling inspection of pipe integrity for improved efficiency of non-destructive testing. Water Science and Technology: Water Supply.

Skinner, B., Vidal-Calleja, T., Valls Miro, J., De Bruijn, F., and Falque, R. (2014). 3D point cloud upsampling for accurate reconstruction of dense 2.5D thickness maps. In Australasian Conference on Robotics and Automation, page 7 . 
Smith, M., Posner, I., and Newman, P. (2010). Efficient non-parametric surface representations using active sampling for push broom laser data. In Robotics: Science and Systems.

Sun, L., Vidal-Calleja, T., and Valls Miro, J. (2015). Bayesian fusion using conditionally independent submaps for high resolution 2.5 D mapping. In IEEE International Conference on Robotic and Automation, pages 3394-3400.

Taheri, H., Qiao, B., and Ghaeminezhad, N. (2015). Kinematic model of a four mecanum wheeled mobile robot. International Journal of Computer Applications, 113:6-9.

Tartakovsky, D. M. and Xiu, D. (2006). Stochastic analysis of transport in tubes with rough walls. Journal of Computational Physics, 217(1):248-259.

Tesch, M., Schneider, J., and Choset, H. (2011). Using response surfaces and expected improvement to optimize snake robot gait parameters. In IEEE/RSJ International Conference on Intelligent Robots and Systems, pages 1069-1074.

Ulapane, N., Alempijevic, A., Vidal-Calleja, T., Valls Miro, J., Rudd, J., and Roubal, M. (2014). Gaussian process for interpreting pulsed eddy current signals for ferromagnetic pipe profiling. In IEEE Conference on Industrial Electronics and Applications, pages 1762-1767.

Ulapane, N., Nguyen, L., Valls Miro, J., and Dissanayake, G. (2017). Designing a pulsed eddy current sensing setup for cast iron thickness assessment. In IEEE Conference on Industrial Electronics and Applications, pages 892-897.

Valls Miro, J., Hunt, D., Ulapane, N., and Behrens, M. (2017). Towards automatic robotic NDT dense mapping for pipeline integrity inspection. In Field and Service Robotics, Results of the 11th International Conference, pages 319-333.

Valls Miro, J., Rajalingam, J., Vidal-Calleja, T., de Bruijn, F., Wood, R., Vitanage, D., Ulapane, N., Wijerathna, B., and $\mathrm{Su}, \mathrm{D}$. (2014). A live test-bed for the advancement of condition assessment and failure prediction research on critical pipes. Water Asset Management International, 10(2):3-8.

Vasudevan, S., Ramos, F., Nettleton, E., Durrant-Whyte, H., and Blair, A. (2009). Gaussian process modeling of large scale terrain. In IEEE International Conference on Robotic and Automation, pages 1047-1053.

Vidal-Calleja, T., Su, D., De Bruijn, F., and Valls Miro, J. (2014). Learning spatial correlations for bayesian fusion in pipe thickness mapping. In IEEE International Conference on Robotics and Automation, pages 683-690.

Xie, L., Scheifele, C., Xu, W., and Stol, K. A. (2015). Heavy-duty omni-directional mecanum-wheeled robot for autonomous navigation: System development and simulation realization. In International Conference on Mechatronics, pages 256-261. 
$\mathrm{Xu}, \mathrm{Z}$., Wu, X., Li, J., and Kang, Y. (2012). Assessment of wall thinning in insulated ferromagnetic pipes using the time-to-peak of differential pulsed eddy-current testing signals. NDT \& E International, 51:24-29. 